\title{
WestVirginiaUniversity
}

THE RESEARCH REPOSITORY @ WVU

Graduate Theses, Dissertations, and Problem Reports

2012

\section{Discrete Damage Mechanics Applied To Transverse Matrix Cracking}

Fernando A. Cosso

West Virginia University

Follow this and additional works at: https://researchrepository.wvu.edu/etd

\section{Recommended Citation}

Cosso, Fernando A., "Discrete Damage Mechanics Applied To Transverse Matrix Cracking" (2012). Graduate Theses, Dissertations, and Problem Reports. 238.

https://researchrepository.wvu.edu/etd/238

This Thesis is protected by copyright and/or related rights. It has been brought to you by the The Research Repository @ WVU with permission from the rights-holder(s). You are free to use this Thesis in any way that is permitted by the copyright and related rights legislation that applies to your use. For other uses you must obtain permission from the rights-holder(s) directly, unless additional rights are indicated by a Creative Commons license in the record and/ or on the work itself. This Thesis has been accepted for inclusion in WVU Graduate Theses, Dissertations, and Problem Reports collection by an authorized administrator of The Research Repository @ WVU. For more information, please contact researchrepository@mail.wvu.edu. 


\title{
Discrete Damage Mechanics Applied To Transverse Matrix Cracking
}

\author{
Fernando A. Cosso
}

Thesis submitted to the Benjamin M. Statler College of Engineering and Mineral Resources at West Virginia University in partial fulfillment of the requirements for the degree of

Master of Science

In

Mechanical Engineering

Ever J. Barbero, Ph.D., Chair

Victor H. Mucino, Ph.D.

Eduardo M. Sosa, Ph.D.

Department of Mechanical and Aerospace Engineering

\author{
Morgantown, West Virginia
}

2012

Keywords: Transverse cracking; Analytical modeling; Computational modeling; Damage mechanics

Copyright 2012 Fernando A. Cosso 


\begin{abstract}
All rights reserved
INFORMATION TO ALL USERS

The quality of this reproduction is dependent upon the quality of the copy submitted.

In the unlikely event that the author did not send a complete manuscript and there are missing pages, these will be noted. Also, if material had to be removed,

a note will indicate the deletion.
\end{abstract}

UMI 1522507

Published by ProQuest LLC (2013). Copyright in the Dissertation held by the Author.

Microform Edition (c) ProQuest LLC.

All rights reserved. This work is protected against

unauthorized copying under Title 17, United States Code

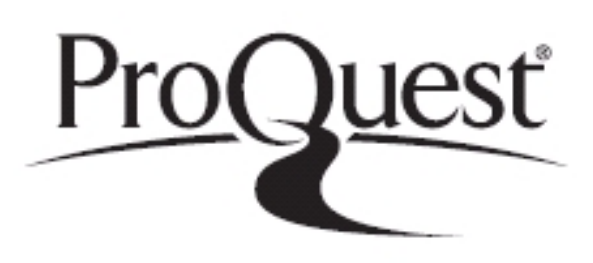

ProQuest LLC.

789 East Eisenhower Parkway

P.O. Box 1346

Ann Arbor, MI $48106-1346$ 


\title{
Abstract \\ Discrete Damage Mechanics Applied To Transverse Matrix Cracking
}

\author{
Fernando A. Cosso
}

A constitutive model to predict the stiffness and coefficient of thermal expansion (CTE) reduction due to transverse matrix cracking in laminated composite structures is implemented as a user subroutine in Abaqus. The assumptions of the model are a symmetric laminate under inplane loads and linear distribution of intralaminar shear stresses. The model was latter validated using 3D finite element analysis (FEA) in a repetitive unit volume for a wide interval of crack densities and for several laminates, and the difference was found to be within 3\%. The FEA, the model and classical laminate theory all converged to the same values of stiffness and CTE for the limiting cases of intact and completely damaged material. The user subroutine was then used to predict the crack density, longitudinal elastic modulus and Poisson's ratio as a function of strain or stress for laminates reported in the literature. For this purpose, the energy release rate, $G_{I c}$, a material property not accessible through experimentation, was adjusted by a minimization algorithm. The influence of the choice of the damage activation function (interacting or not interacting) in the convergence was studied as well, and it was found that the non-interacting damage function converges faster to the desired value of energy release rate, when compared to the interacting one. Overall, the model is able to predict well damage initiation and evolution for e-glass and carbon fiber composites of arbitrary symmetric stacking sequence. 


\section{Contents}

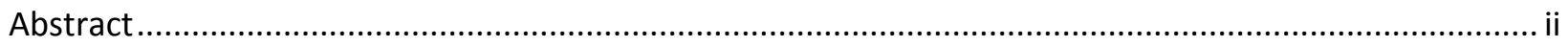

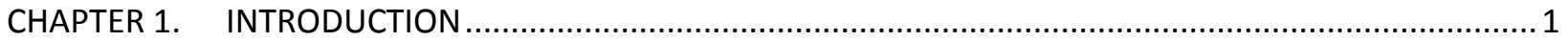

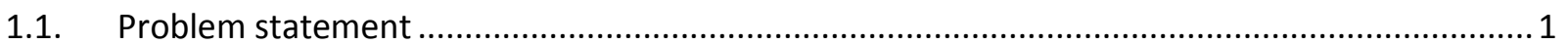

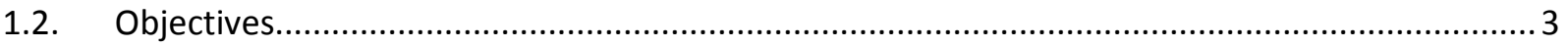

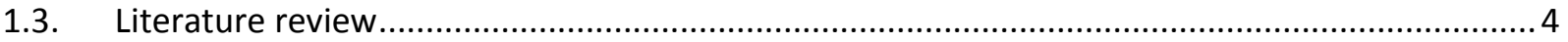

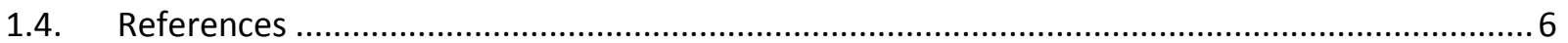

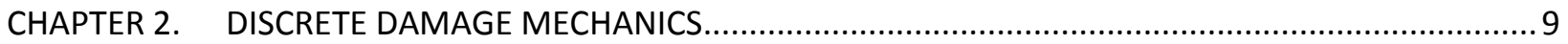

2.1. Material Properties vs. Crack density …............................................................................. 9

2.1.1. Shear Lag Equations in Matrix Form .......................................................................... 11

2.1.2. Solutions of the Equilibrium Equation ........................................................................... 12

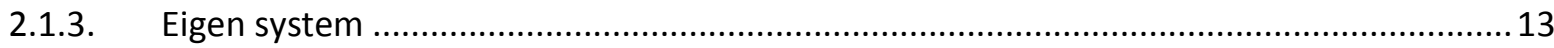

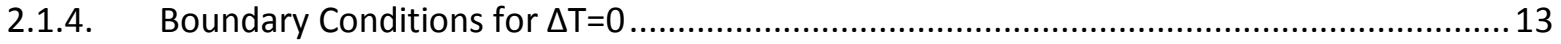

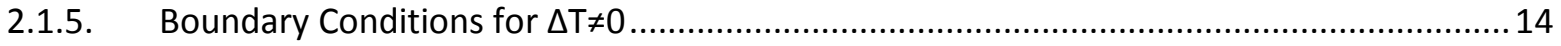

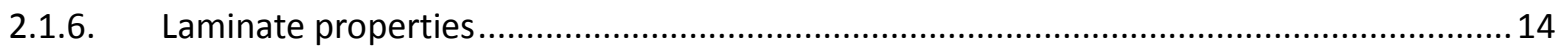

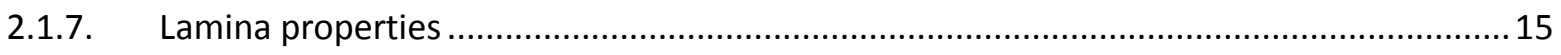

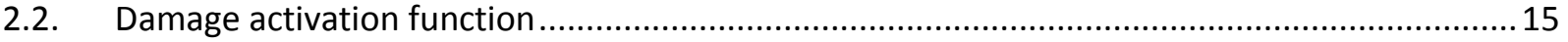

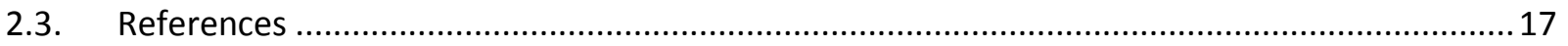

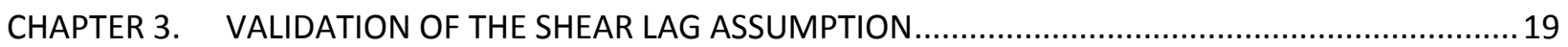

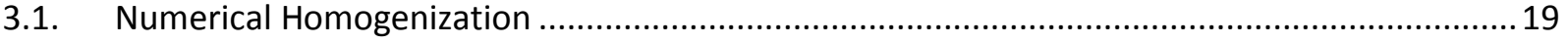

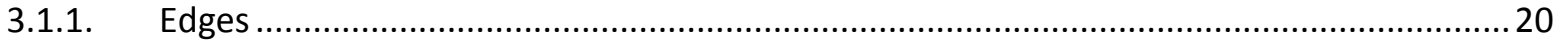

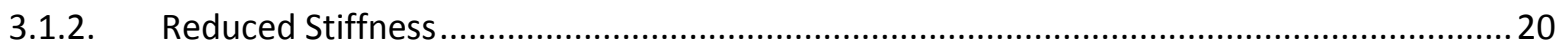

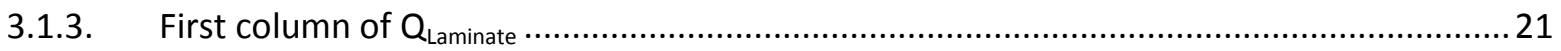

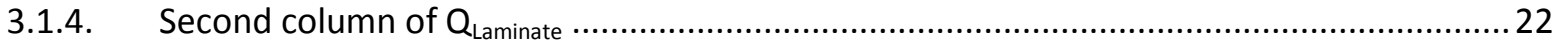

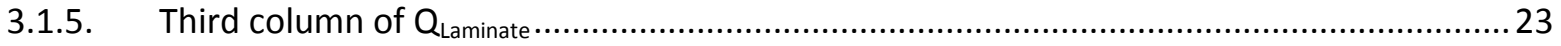

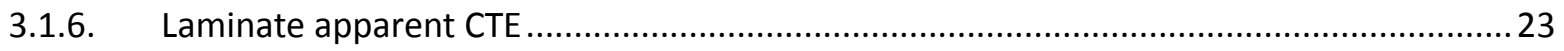

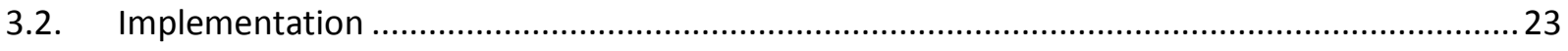

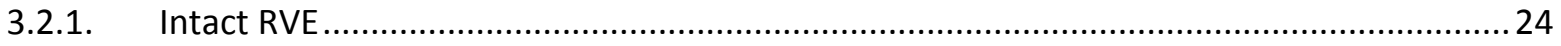

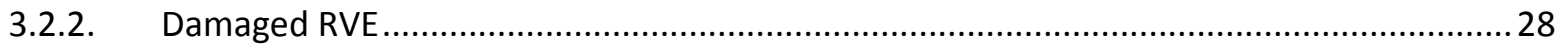

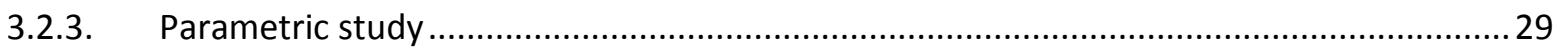

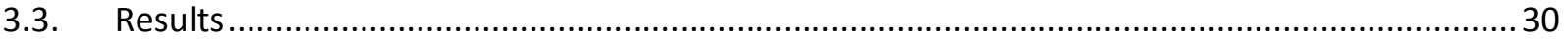

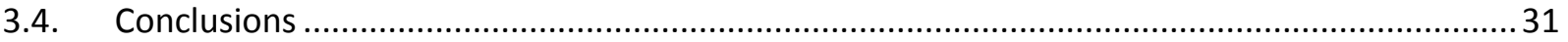




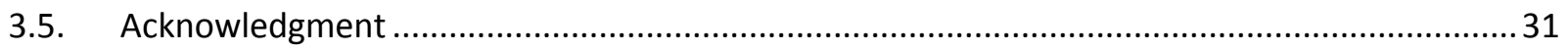

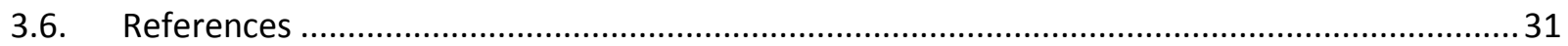

CHAPTER 4. DETERMINATION OF G

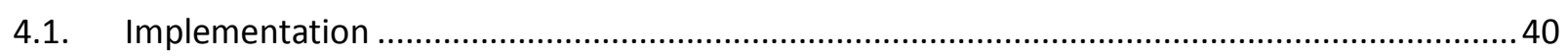

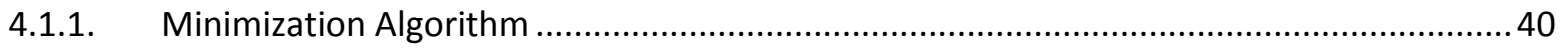

4.1.2. Measurement of the distance between points .............................................................. 40

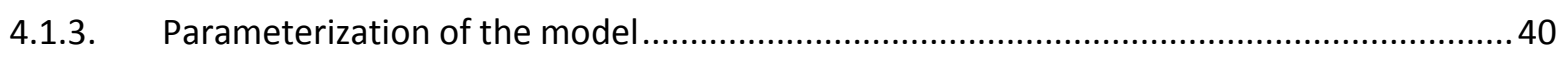

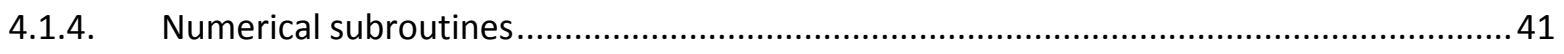

4.1.5. Need for a client-server architecture......................................................................... 41

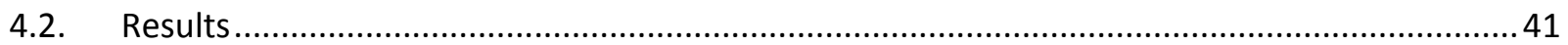

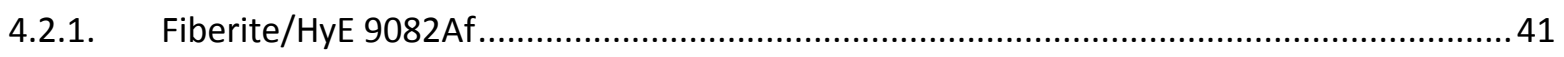

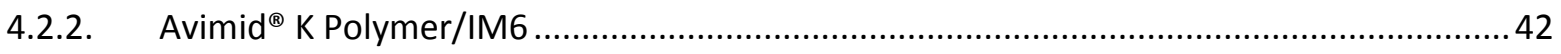

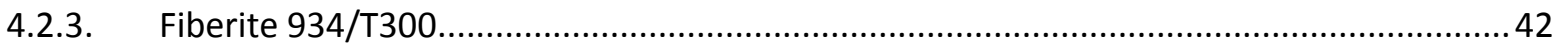

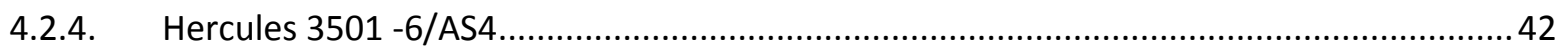

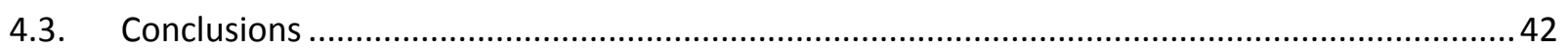

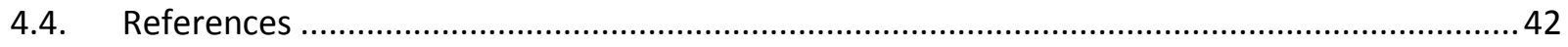

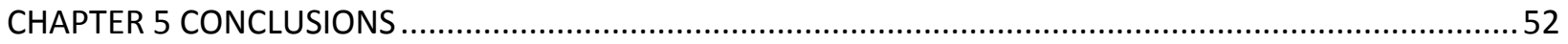

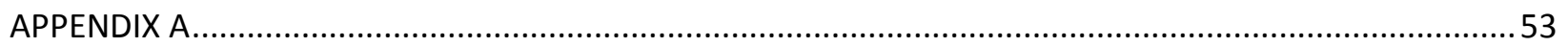




\section{Figures}

Figure 1.1 Matrix cracks in a $\left[030_{2}\right.$ 90]s laminate for $1.1 \%$ of strain, The 0 plies are horizontal and the 90 plies are vertical, where cracks are visible. Reprinted from International Journal of Solids and Structures, 42/9-10, Yokozeki, T., Aoki, T., Ishikawa, T., Consecutive matrix cracking in contiguous plies of composite laminates, 2796, Copyright 2005, with permission from Elsevier. 3

Figure $1.2 \mathrm{CDM}$ approach. (a) Unstressed material. (b) Stressed material. (c) Effective configuration. Barbero, E. J., Reproduced from Finite Element Analysis of Composite Materials, Figure 8.1, p. 192, Copyright 2008, with permission from Dr. E. J. Barbero.

Figure 1.3 Crack Opening Displacement. Reprinted from Engineering Fracture Mechanics, 60/2, A. Poursartip,A. Gambone,S. Ferguson,G. Fernlund, In-situSEM measurements of crack tip displacements in composite laminates to determine localGin mode I and II, 173-185, Copyright 1998, with permission

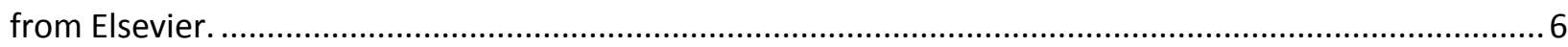

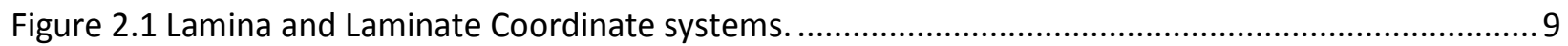

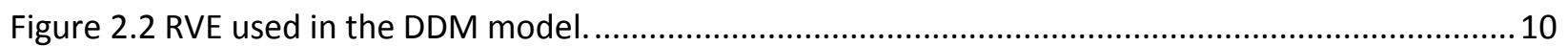

Figure 2.3 Assumed distribution of intralaminar shear stress for lamina i........................................... 10

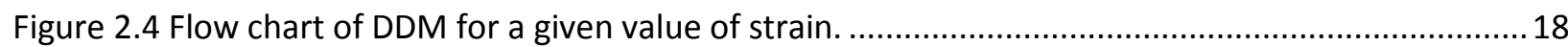

Figure 3.1 Top view of the RVE. Faces are shown as lines and edges are shown as vertices....................20

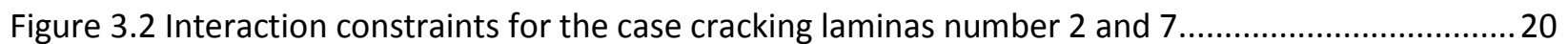

Figure 3.3 Percent error vs. \# Elements for C3D8 and C3D20 element types......................................... 32

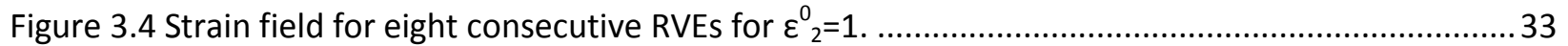

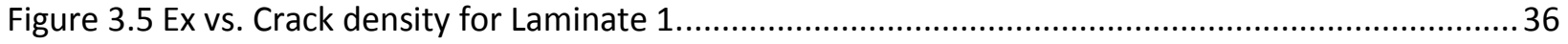

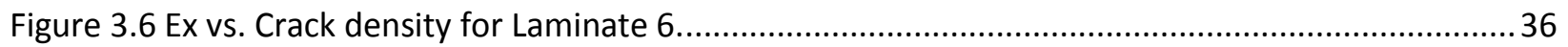

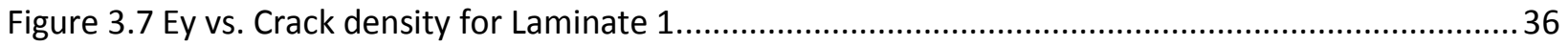

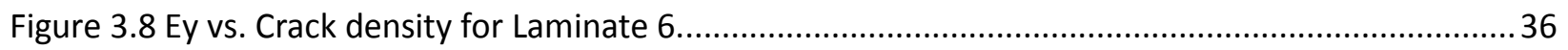

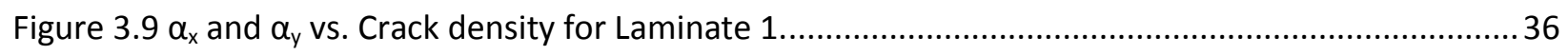

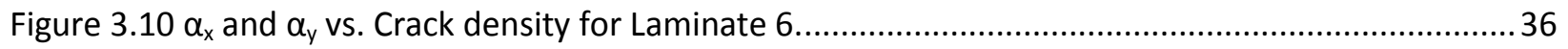

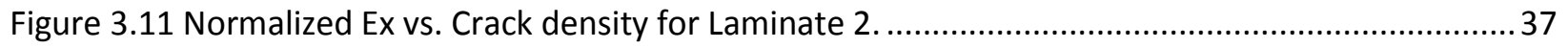

Figure 3.12 Normalized Poisson's Ratio vs. Crack density for Laminate 2. ........................................... 37

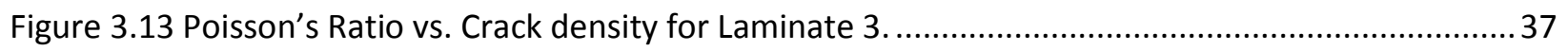

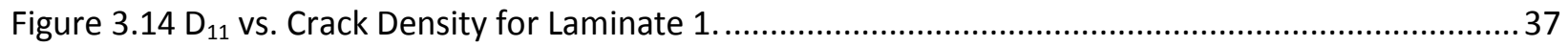

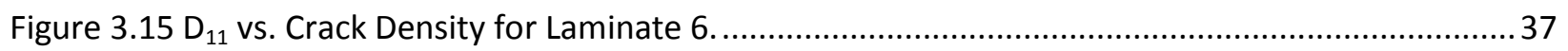

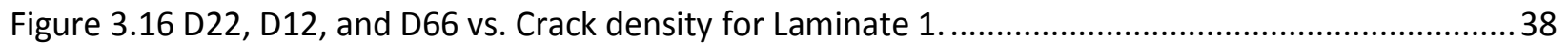

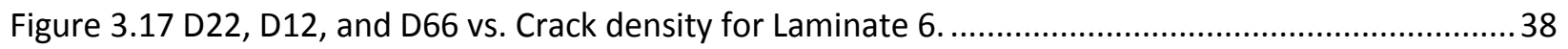

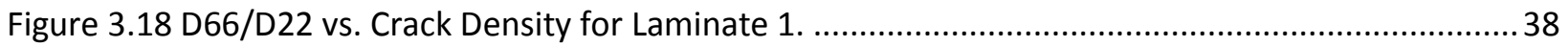

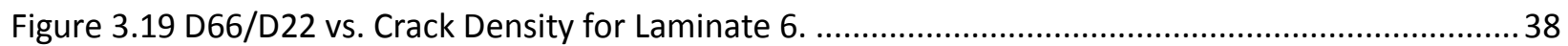

Figure 4.1 Error vs. GIIC normalized by GIC for the interacting and non-interacting damage functions for Laminate 1 (cracks propagate in mode I). The non-interacting damage function converges faster to the minimum error. Grayed zone correspond to hypothetical values, experimentally GIIC $>$ GIC ...............39 Figure 4.2 Force vs. Displacement for Laminate 2. The solid line is independent (up to single precision) of element type and number. 
Figure 4.3 Generic $\mathrm{P}$ vs. X plot. Squares are experimental points and circles are Abaqus points. Dashed line is the spline that allows for the evaluation of $\mathrm{P}^{\mathrm{DDM}}$ at the same value of experimental $\mathrm{x} . \ldots \ldots \ldots \ldots \ldots . . . . . . .42$

Figure 4.4 Flowchart for the Abaqus, Matlab client server tcp/ip communication. ............................... 45

Figure 4.5 Crack Density (P) vs. Strain ( $x$ ) for Laminate 1. Error calculated as in (4.1) is 0.038991 . Squares

are experiment set 1 , circles are experiment set 2, solid line is the DDM prediction...............................46

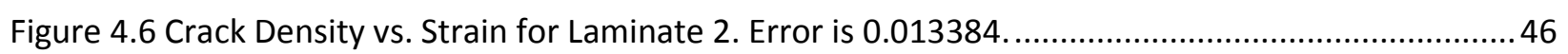

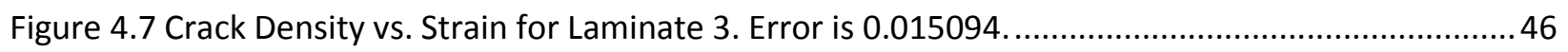

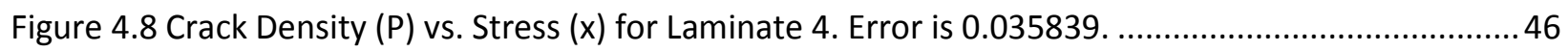

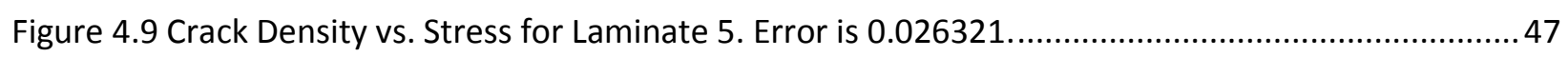

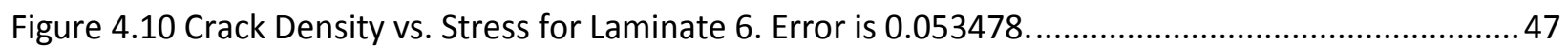

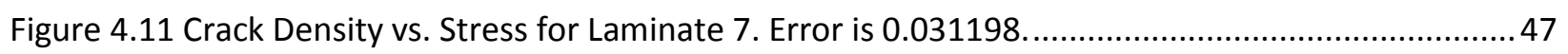

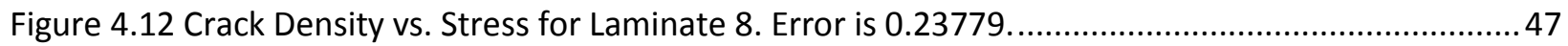

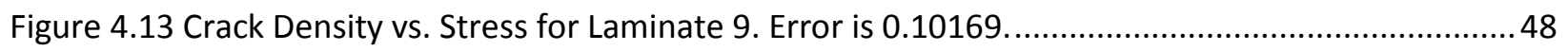

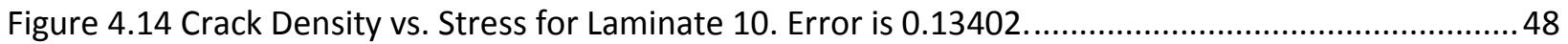

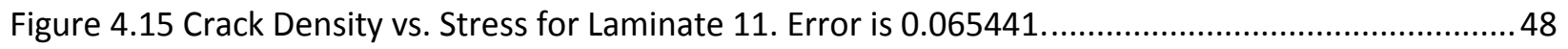

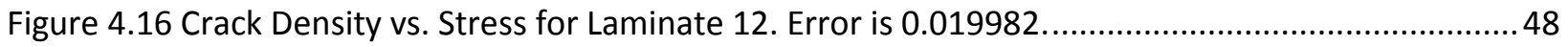

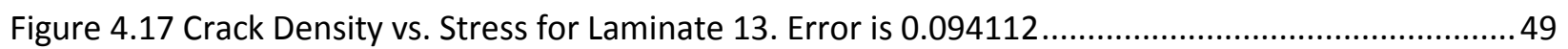

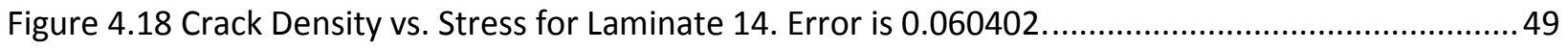

Figure 4.19 Normalized Ex vs. Strain for Laminate 15. Error is 0.010807.............................................. 49

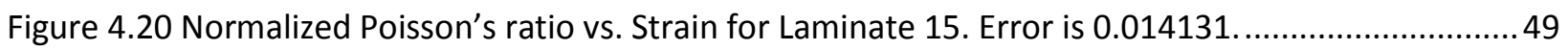

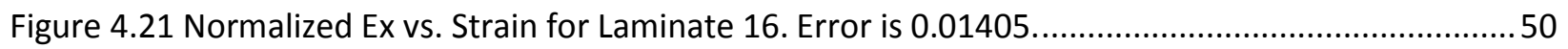

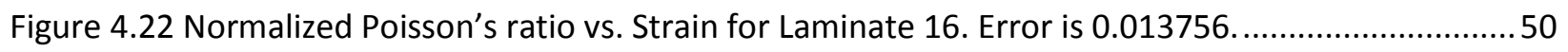

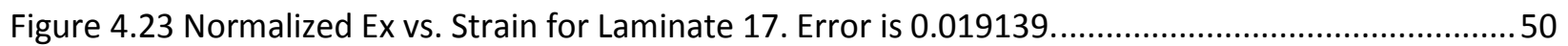

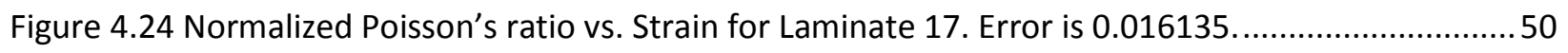

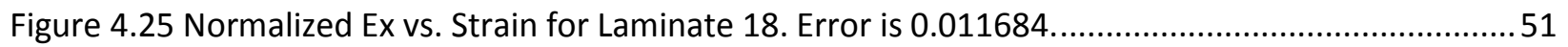

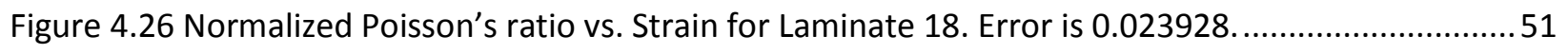

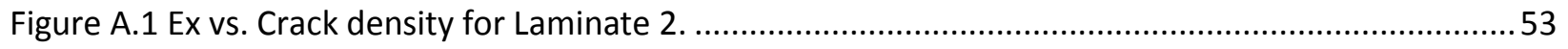

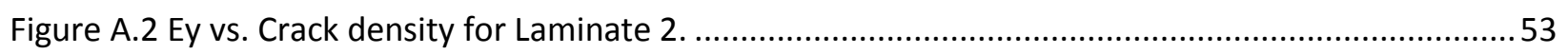

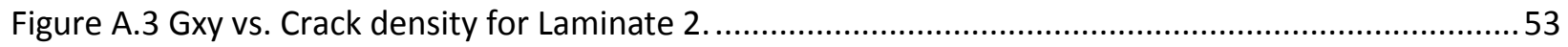

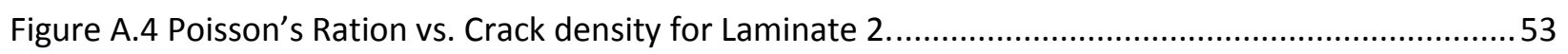

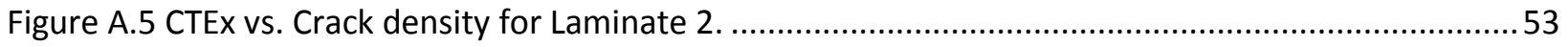

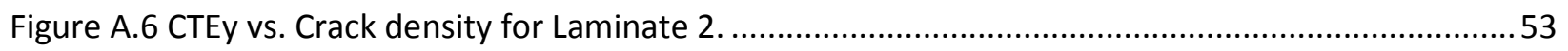

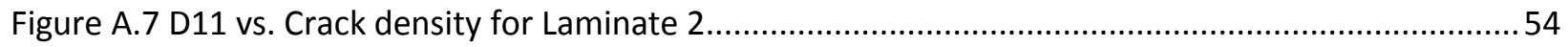

Figure A.8 D12, D66 and D22 vs. Crack density for Laminate 2 ........................................................54

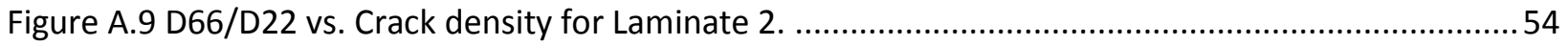

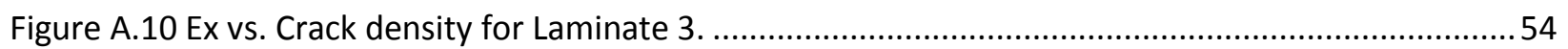

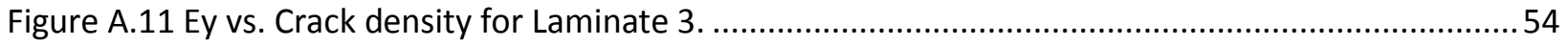

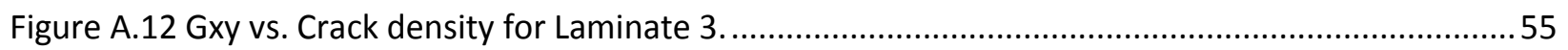

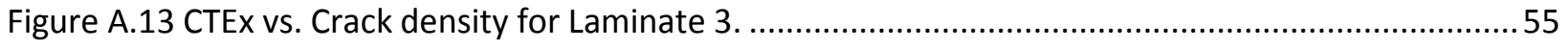

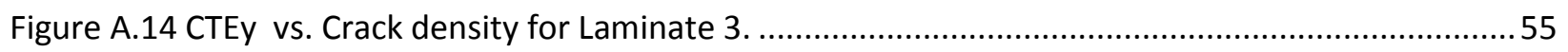

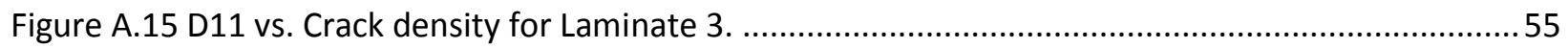

Figure A.16 D22,D66 and D12 vs. Crack density for Laminate 3.......................................................5 55 
Figure A.17 D66/D22 vs. Crack density for Laminate 3. .................................................................. 55

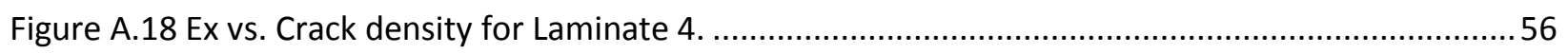

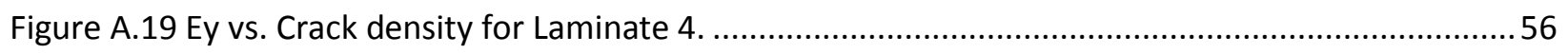

Figure A.20 Gxy vs. Crack density for Laminate 4...........................................................................5 56

Figure A.21 Poisson's Ration vs. Crack density for Laminate 4 .............................................................56

Figure A.22 CTEx vs. Crack density for Laminate 4 .............................................................................5

Figure A.23 CTEy vs. Crack density for Laminate 4 .........................................................................56

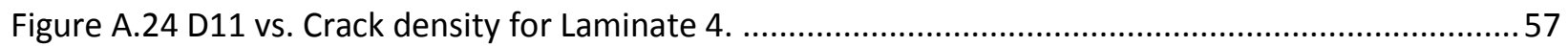

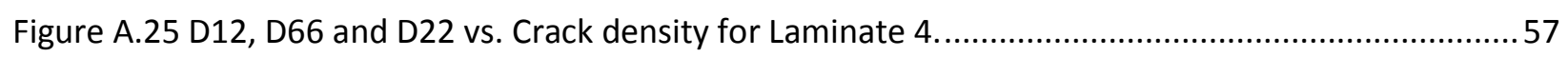

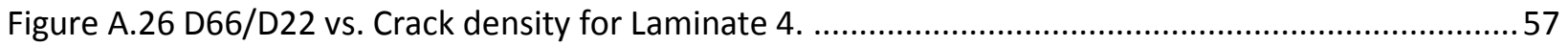

Figure A.27 Ex vs. Crack density for Laminate 5 ......................................................................... 57

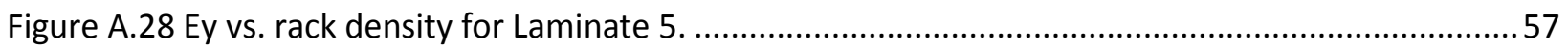

Figure A.29 Gxy vs. Crack density for Laminate 5......................................................................... 58

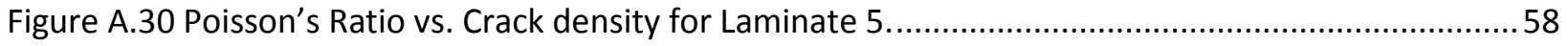

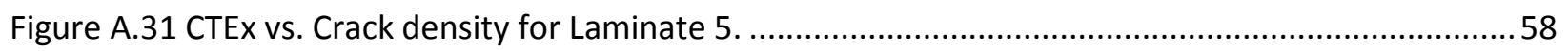

Figure A.32 CTEy vs. Crack density for Laminate 5 . .......................................................................... 58

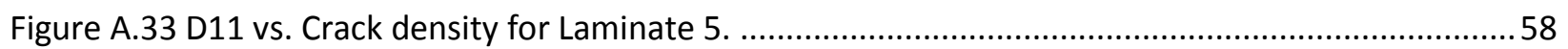

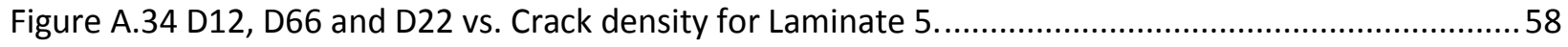

Figure A.35 D66/D22 vs. Crack density for Laminate 5. ..........................................................................59

\section{Tables}

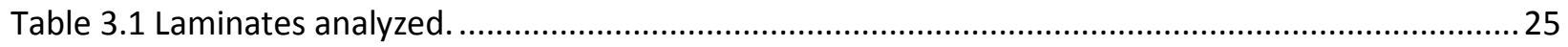

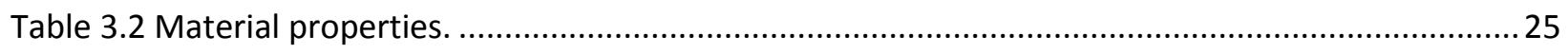

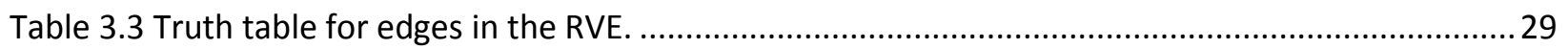

Table 3.4 Laminate 1 averaged strains in the intact RVE for the three boundary condition cases............29

Table 4.1 GIC obtained by the minimization algorithm for the materials analyzed................................ 40

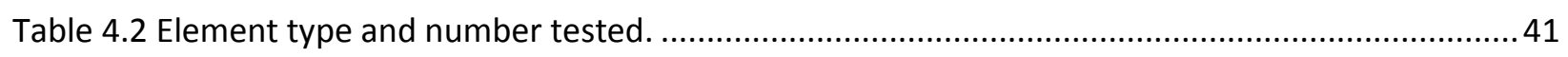




\section{CHAPTER 1. INTRODUCTION}

\subsection{Problem statement}

The definition of a composite material varies among different authors, but it typically involves the description of a composite material as the combination of at least two different materials, either naturally occurrently in nature or manmade to fulfill a requirement that would not be possible any other way.

Composite materials are composed of two main components: matrix and reinforcement. The matrix is the component that holds the composite together while the reinforcement provides for the load resistance. The matrix is commonly the mayor component by volume, but not always.

In an effort to characterize the wide diversity of composites, it is sometimes useful to divide the composites by the type of matrix as:

- Metal Matrix Composites (MMC)

- Ceramic Matrix Composite (CMC)

- $\quad$ Polymer Matrix Composite (PMC)

also, by the layout of the reinforcements:

- Fibers

- Continuous long fibers

- Unidirectional fiber orientation

- Fabrics

- Random orientation

- Discontinuous fibers

- Random orientation

- Preferential orientation

- Particles and Whiskers

- Random orientation

- Preferential orientation

And by the laminate configuration:

- Bulk composites: The structure cannot be treated as a laminate

- Unidirectional lamina: All the laminas are oriented in the same direction

- Laminate: Several, very thin, laminas with different orientations are glued together to form a panel.

- Sandwiches: A very lightweight material separates two thin but stiff layers giving the composite high bending stiffness. 
Composite materials are heterogeneous by definition. This inhomogeneity complicates the design and analysis of composite structures. At the macroscopic level, it is useful to forget about the microscopic details of the material and treat the composite as a homogeneous material. This is possible by homogenizing the properties of the constituent materials to come up with equivalent properties for the composite. At the basic level this is done by averaging. Take the density as an example. The density of the composite is a weighted average of the density of its constituents:

$$
\delta_{\mathrm{c}}=\sum_{i=1}^{N} \delta_{i} V_{i}
$$

Where $\delta_{c}$ is the composite density, and $\delta_{i}, V_{i}$ are the ith component's density and volume fraction respectively.

Micromechanics is the field that studies precisely that: how to predict the properties of a composite. Other properties need further refinement that what we have seen in (1.1) to be accurately predicted by the micromechanics models.

The need for models that can predict properties based on the geometry and character of the constitutive materials is evident if we consider that: (a) composites are generally anisotropic (the value of the properties change with the orientation) and (b) the properties change significantly by changing the geometry and relative amounts of the constituent materials. It would be very costly to explore these two aspects solely with experiments; hence any relation that can be developed from analytical or numerical models is generally met with interest.

Macromechanics predicts the behavior of the composite as whole. For laminated composites, the constitutive relationships of the whole laminate are given by the Classical Laminate Theory (CLT). CLT is useful to relate the strains to the loads provided the stiffness and coefficient of thermal expansion (CTE) of each lamina that is contained in the laminate are given.

One type of property that is especially useful to the designer is the strength of the composite. That is, to what level of load the composite is expected to fail. This type of property is especially difficult to predict due to the stochastic nature of the events that lead to failure. The particulars of the manufacturing process (fiber alignment, voids, residual stresses) play a significant role in determining the composite capacity to resist loads, along with the nature of the loads that are applied (Variation, dynamics, direction, etc.). Different modes of failure can be recognized that occur in composites and a model that takes into account the specific of that mode needs to be developed.

Oftentimes, the failure mode is not critical to the structural stability, that is, the composite degrades but is still capable of handling the loads. When this happens, it is extremely important to be able to predict the evolution of this condition because it can progress up to a point where other fatal modes are triggered and the composite breaks.

Laminated composite materials damage occurs in two levels: inter-lamina damage and intra-lamina damage. Inter-lamina damage is the damage that appears in the interface between two laminas. Delamination is a common form of inter-lamina damage and is a very serious problem. Intra-lamina 
damage refers to the occurrence of cracks through the matrix, breakage of the fibers or any other phenomena that appears in the interior of a lamina.

Figure 1.1 shows the cracks in a $\left[030_{2}\right.$ 90]s laminate, the cracks run along the fiber direction of each ply that is cracking $\left(30^{\circ}\right.$ and $90^{\circ}$ in the figure) and are equally spaced. Matrix cracking affects the performance of the composite because the stiffness is reduced, the cracks act as stress concentrators where delamination occurs and can lead to leaking.

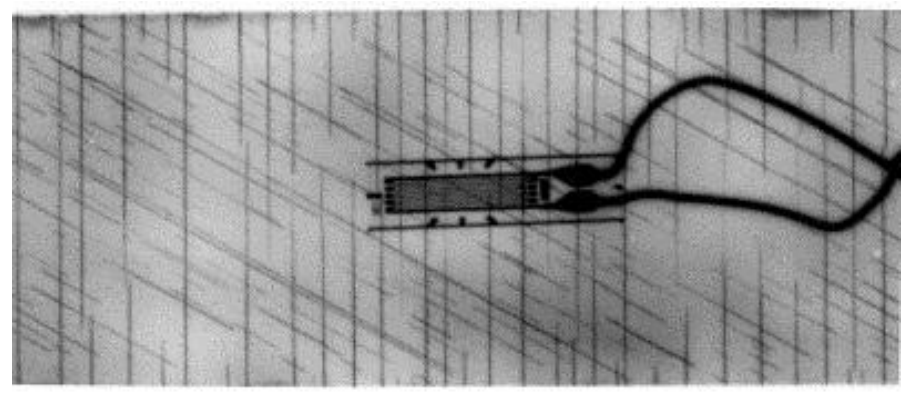

Figure 1.1 Matrix cracks in a $\left[03_{2}\right.$ 90]s laminate for $1.1 \%$ of strain, The 0 plies are horizontal and the 90 plies are vertical, where cracks are visible. Reprinted from International Journal of Solids and Structures, 42/9-10, Yokozeki, T., Aoki, T., Ishikawa, T., Consecutive matrix cracking in contiguous plies of composite laminates, 2796, Copyright 2005, with permission from Elsevier.

The prediction of damage initiation and evolution is the field of study of damage mechanics. The present work implements a model that investigates the damage of laminate composites when transverse cracks occur. The model assumes a symmetric laminate and in-plane loads with no bending moments applied.

\subsection{0bjectives}

The objective of the model is to be able to predict damage onset and track the reduction of stiffness and coefficient of thermal expansion as a function of crack density and loading conditions. For this purpose, a discrete damage mechanics (DDM) approach is employed to solve for the displacement fields and a fracture mechanics equation is used as a damage evolution criteria. The model is built in a representative volume element (RVE), which is the smallest portion of the material that contains all the peculiarities of the microstructure, where only one lamina is considered cracking at a time. The other laminas can crack too, but are considered homogenized during the analysis. Once the crack density for the cracking lamina is such that equilibrium is reached, the model homogenizes the lamina and continues to evaluate the following lamina and so on. The model is explained in detail in Chapter 2.

Chapter 3 involves the finite element analysis (FEA) validation of the approximate solution for the equilibrium equations. Carbon/Epoxy and Glass/Epoxy laminates with off-axis laminas were analyzed and the laminate stiffness and CTE versus crack density were compared for DDM, FEA and in the limiting cases Classical Laminate Theory. Experimental data was also used to compare the FEA and DDM predictions. The FEA model uses linear elasticity and a custom implementation of periodic boundary conditions. Additionally, a convergence study was conducted to determine the mesh size. The equivalent stiffness and CTE of the cracking lamina was also assessed by the FEA model and compared to the DDM prediction. It is important to note that in this chapter nothing is done with regard to damage 
onset and evolution. The objective of this chapter is to validate the functional relationship between stiffness and CTE vs. crack density. That is, given a known value of crack density, what would be the stiffness and CTE of the laminate?

Chapter 4 deals with the damage activation function and how the crack density changes when the laminate is subjected to an increasing load. Experimental results from the literature are employed to study the effect of the choice of the damage activation function and to compare the DDM prediction. A fitting procedure is used to find the only parameter required by DDM and is also explained in detail.

Finally, once the damage due to matrix cracking has been characterized, it is desired to build up to predict damage due to delamination, since delamination tends to occur whenever matrix cracks are present. In this sense, this work is the foundation for future research in the area of delamination.

\subsection{Literature review}

Transverse matrix cracking of laminated composites has attracted the attention of the research community and has extensively been studied. Different approaches have been employed to analyze this phenomenon.

Continuum Damage Mechanics (CDM) is a phenomenological approach that represents the damage in terms of state variables without explicitly tacking into account the discrete cracks (see Figure 1.2). The progression of the damage follows some postulated equations that require additional parameters that have to be fitted using experimental data or a mechanistic model. For a review of CDM consult the work of Talreja [1] and Barbero [2] for a set of examples. Additional works that use CDM can be found in [3-5].

Micromechanics of Damage (MMD) models take into account the cracks in the laminate in an explicit microscopically fashion. The elasticity equations are stated in a region close to the cracks and solved for with some approximation as to the functional form of the stresses. The strain and stress obtained by this analysis are referred to as "micro" strain/stress as opposed to the averaged or homogenized strain/stresses that coincide with the imposed load and blur the variations that occur between cracks. This approach is not exclusive of damage mechanics, but has also been used in other areas of solid state physics, for an excellent discussion of this microscopic/macroscopic treatment of the electric vector fields see the classical book by Ashcroft [6].

The model implemented in this work uses MMD for the treatment of a cracking lamina at a time and CMD to incorporate the damage of the remaining laminas in a homogenized way. The model can be found in [7], which is a simplification of the model developed by Yokozeki and Aoki [8] that uses oblique coordinate system and can only handle at most two cracking laminas. The model was implemented along with a three node shell element formulation for ANSYS in [9].

The Crack Opening Displacement (COD) method is based on the theory of elastic bodies with voids by Kachanov [10] and derives a functional relationship of thermo mechanical properties versus crack opening displacement, that is, the relative displacement of the two faces of the crack (see Figure 1.3). 
Once this relationship is known, the crack opening displacement is correlated to the external load or strain by means of finite element simulations or known fracture mechanics solutions. Gudmundson and Adolfsson [11-14] built a model based on COD which was later reproduced by Adumitroaie [15]. Lundmark and Varna correlated the crack face sliding displacement to the thickness ratio and the inplane shear stiffness in [16] and used it along the relationship between COD and ply properties, laminate layup and crack density as input to a COD model in [17].

Abaqus ${ }^{1}$ implements a progressive damage method developed originally by Camanho and Davila in [18] that requires additional experimental strength values as input for the Hashin failure criteria [19] used for determining the damage onset. Once damage has initiated the evolution is controlled by the energy dissipation in an equivalent displacement vs. equivalent stress space that has to be provided to the model but it is not clear how to obtain this parameter.

There are some plugins for Abaqus that also address the problem of damage in composite materials, such as $[20,21]$ but they are not free and the specifics of the implementation are proprietary information.

Finite element simulations have been performed either to validate the analytical models, or to provide input for the models, but to the best knowledge of the author, no simulations have extensively been used to predict the thermal expansion coefficient of the laminate for laminates other than [0 90]s.

Gudmundson and Adolfsson used Abaqus to simulate cracks in off-axis plies but the construction of the cell imposed geometric restrictions in the crack densities of the off-axis plies [14,22]. Lundmark and Varna were able to calculate $G_{12}$ in [16] and $E_{2}$ in [17] for symmetric laminates.

Yokozeki et al. used FEA simulations to investigate the energy release rate for two cases: Existing crack propagation and new crack in between existing cracks [23-25].

The present work calculates using FEA the lamina stiffness and CTE for a wide range of crack densities, something that has not been covered in the previous works.

\footnotetext{
${ }^{1}$ Abaqus is a registered trademark of Dassault Systèmes.
} 


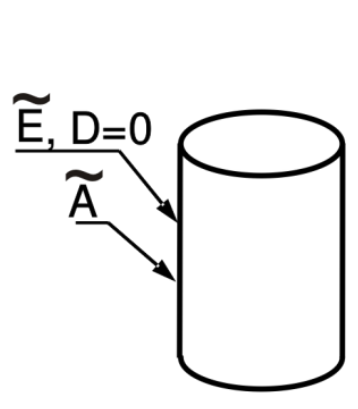

(a)

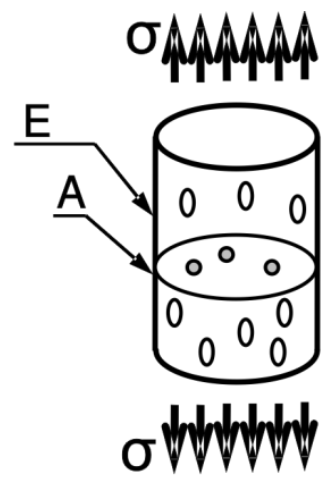

(b)

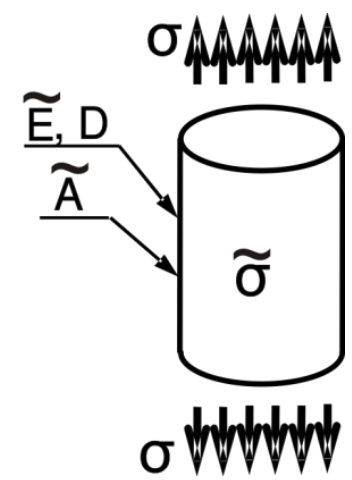

(c)

Figure 1.2 CDM approach. (a) Unstressed material. (b) Stressed material. (c) Effective configuration. Barbero, E. J., Reproduced from Finite Element Analysis of Composite Materials, Figure 8.1, p. 192, Copyright 2008, with permission from Dr. E. J. Barbero.

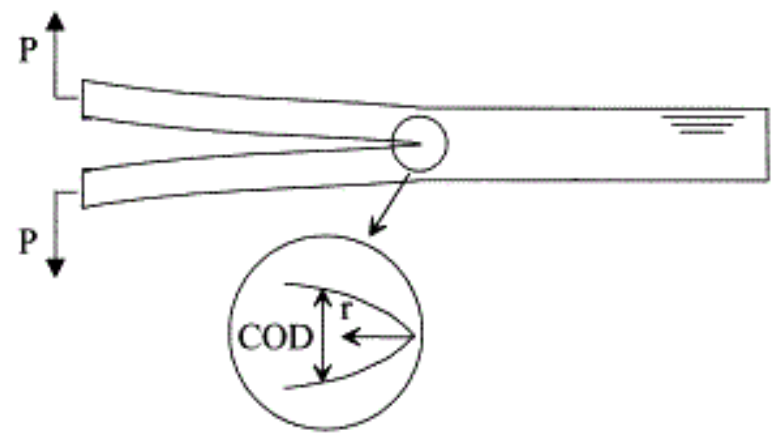

Figure 1.3 Crack Opening Displacement. Reprinted from Engineering Fracture Mechanics, 60/2, A. Poursartip,A. Gambone,S. Ferguson,G. Fernlund, In-situSEM measurements of crack tip displacements in composite laminates to determine localGin mode I and II, 173-185, Copyright 1998, with permission from Elsevier.

\subsection{References}

[1] R Talreja, Damage characterization by internal variables, Damage mechanics of composite materials, Elsevier, Amsterdam [The Netherlands], 1994, pp. 306.

[2] EJ Barbero, Finite element analysis of composite materials, CRC Press, Boca Raton, 2008.

[3] P Maimí, PP Camanho, JA Mayugo, CG Dávila. A continuum damage model for composite laminates: Part I - Constitutive model, Mech.Mater. 39 (2007) 897-908.

[4] P Maimí, PP Camanho, JA Mayugo, CG Dávila. A continuum damage model for composite laminates: Part II - Computational implementation and validation, Mech.Mater. 39 (2007) 909-919.

[5] S Li, SR Reid, PD Soden. A continuum damage model for transverse matrix cracking in laminated fibre-reinforced composites, Philosophical Transactions of the Royal Society A: Mathematical, Physical and Engineering Sciences. 356 (1998) 2379-2412.

[6] NW Ashcroft, ND Mermin, Solid state physics, Saunders College, Philadelphia, 1976. 
[7] EJ Barbero, DH Cortes. A mechanistic model for transverse damage initiation, evolution, and stiffness reduction in laminated composites, Composites Part B: Engineering. 41 (2010) 124-132.

[8] T Yokozeki, T Aoki. Overall thermoelastic properties of symmetric laminates containing obliquely crossed matrix cracks, Composites Sci.Technol. 65 (2005) 1647-1654.

[9] G Sgambitterra, A Adumitroaie, EJ Barbero, A Tessler. A robust three-node shell element for laminated composites with matrix damage, Composites Part B: Engineering. 42 (2011) 41-50.

[10] M Kachanov, Elastic Solids with Many Cracks and Related Problems, Advances in Applied Mechanics, Elsevier, (1993), pp. 259-445.

[11] P Gudmundson, O Soren. First order analysis of stiffness reduction due to matrix cracking, J.Composite Mater. 26 (1992) 1009-1030.

[12] P Gudmundson, S Östlund. Prediction of thermoelastic properties of composite laminates with matrix cracks, Composites Sci.Technol. 44 (1992) 95-105.

[13] P Gudmundson, Z Weilin. An analytic model for thermoelastic properties of composite laminates containing transverse matrix cracks, Int.J.Solids Structures. 30 (1993) 3211-3231.

[14] E Adolfsson, P Gudmundson. Thermoelastic properties in combined bending and extension of thin composite laminates with transverse matrix cracks, Int.J.Solids Structures. 34 (1997) 2035-2060.

[15] A Adumitroaie, Initiation and Evolution of Matrix Cracking in Non-Symmetric Laminates under inPlane and Flexural Loading, Ph.D. Dissertation, West Virginia University. (2012).

[16] P Lundmark, J Varna. Crack face sliding effect on stiffness of laminates with ply cracks, Composites Sci.Technol. 66 (2006) 1444-1454.

[17] P Lundmark, J Varna. Constitutive relationships for laminates with ply cracks in in-plane loading, Int J Damage Mech. 14 (2005) 235-259.

[18] P Camanho, C Davila, Mixed-Mode Decohesion Finite Elements for the Simulation of Delamination in Composite Materials, NASA/TM-2002-211737. (2002).

[19] Z Hashin. Failure Criteria for Unidirectional Fiber Composites, J.Appl.Mech.Trans.ASME. 47 (1980) 329-334.

[20] GENOA Virtual Testing \& Analysis Software, Alpha STAR Corporation, (2012).

[21] Helius:MCT Composite Materials Analysis Software, Firehole Composites, (2012).

[22] E Adolfsson, $P$ Gudmundson. Matrix crack induced stiffness reductions in $[(0 \mathrm{~m} / 90 \mathrm{n} /+\theta \mathrm{p} /-\theta \mathrm{q}) \mathrm{s}$ ] M composite laminates, Composites Engineering. 5 (1995) 107-123. 
[23] T Yokozeki, T Aoki, T Ishikawa. Transverse crack propagation in the specimen width direction of CFRP laminates under static tensile loadings, J.Composite Mater. 36 (2002) 2085-2099.

[24] T Yokozeki, T Aoki, T Ishikawa. Consecutive matrix cracking in contiguous plies of composite laminates, Int.J.Solids Structures. 42 (2005) 2785-2802.

[25] T Yokozeki, T Aoki, T Ogasawara, T Ishikawa. Effects of layup angle and ply thickness on matrix crack interaction in contiguous plies of composite laminates, Composites Part A: Applied Science and Manufacturing. 36 (2005) 1229-1235. 


\section{CHAPTER 2. DISCRETE DAMAGE MECHANICS}

\subsection{Material Properties vs. Crack density}

Discrete damage mechanics refers to the use of discrete fracture mechanics to predict damage initiation and evolution [1].

This model considers a symmetric laminate under in-plane load. Each lamina in the laminate is susceptible to matrix cracking. The damage activation function $g(\lambda, \epsilon, \Delta T)$, discussed in detail in 2.2, decides whether this happens or not, where $\lambda$ is the crack density, $\epsilon$ is the strain and $\Delta T$ is the temperature difference between the reference temperature and the operation temperature.

The loads are incremented from zero up to a certain point in finite increments. In each load step and for each lamina, the damage activation function is calculated. If $g(\lambda, \epsilon, \Delta T)<0$, then no damage occurs and the next lamina is analyzed until all the laminas have been analyzed. Then, the load is further incremented. If $g(\lambda, \epsilon)>0$, then damage occurs in a particular lamina. A return mapping algorithm is then used to determine the new crack density. As the result of this increase in crack density, there is degradation of elastic properties that can be calculated also.

The lamina coordinate system is denoted by $x_{1}-x_{2}-x_{3}$ and the laminate coordinate system by $x-y-z$ as illustrated in Figure 2.1. Contracted notation [2] is used for stresses and strains in lamina coordinate system. The displacement is described by the three components $u, v$ and $w$.

\section{Laminate Coordinate System}

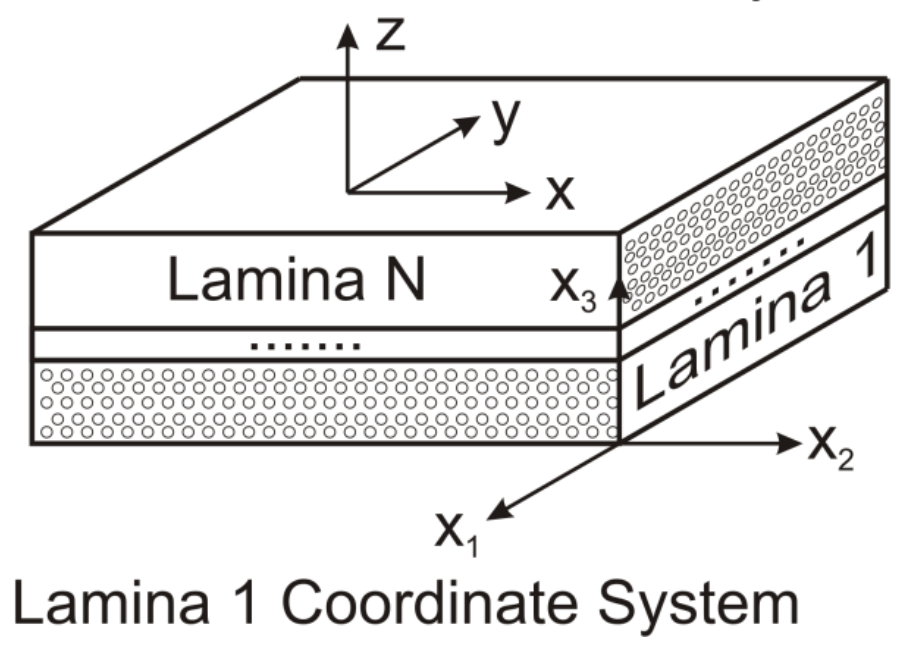

Figure 2.1 Lamina and Laminate Coordinate systems.

The representative volume element (RVE) used in this analysis is delimited by the bottom-surface and mid-surface of the laminate (bottom and top surfaces in Figure 2.2), the surfaces of two consecutives cracks, and a unit length in the direction of the fibers. The length of the RVE is $2 I$ and it is related to the crack density as $2 \mathrm{l}=1 / \lambda$. 


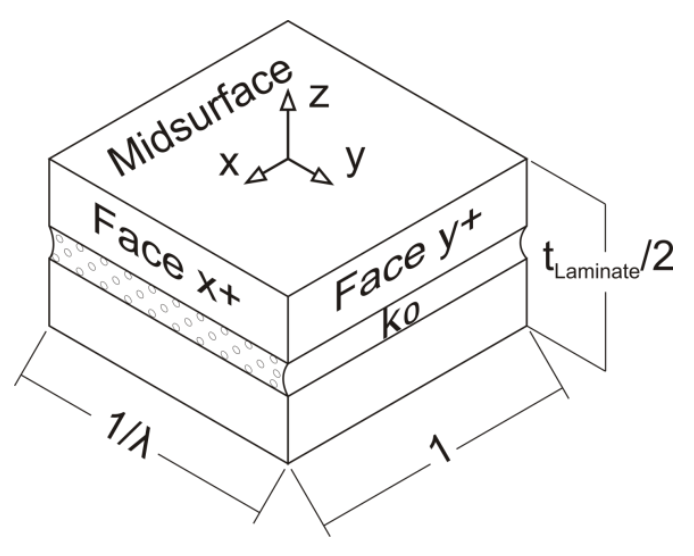

Figure 2.2 RVE used in the DDM model.

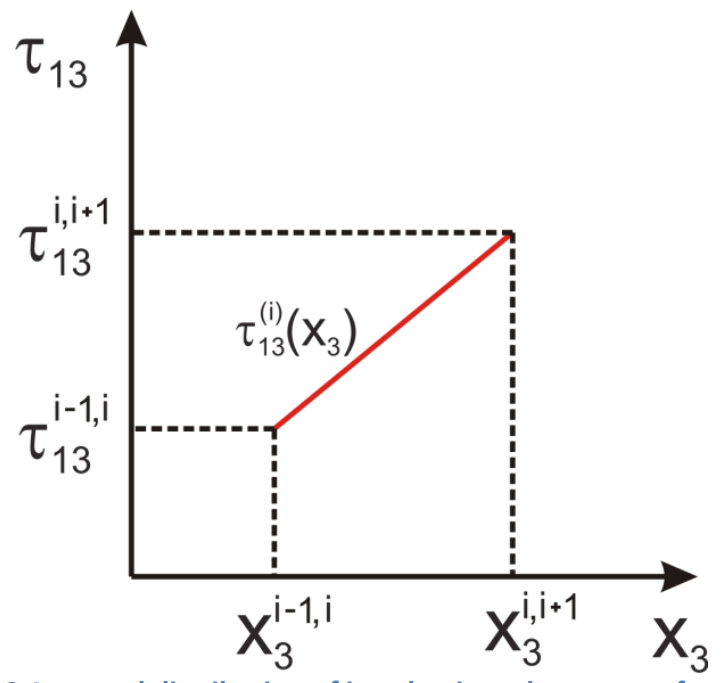

Figure 2.3 Assumed distribution of intralaminar shear stress for lamina i. Since the laminate is symmetric and there are no bending moments applied to the laminate

$$
\frac{\partial w^{(i)}}{\partial x_{1}}=\frac{\partial w^{(i)}}{\partial x_{2}}=0
$$

where the superscript (i) refers to the ith lamina.

The bottom and top surfaces of the laminate are stress-free and the laminate is thin enough as to consider that $\sigma_{3}^{(i)}=0$ (plane stress condition).

The thickness average of any mechanical variable is defined as

$$
\widehat{\phi}=\frac{1}{h} \int_{h} \phi d x_{3}
$$

Where $\phi$ can be any parameter such as $\sigma, \epsilon, \mathrm{Q}$, etc.

This definition is useful for obtaining the overall reduced stiffness properties based on the averaged displacements.

For the cracking lamina $k$, the constitutive equation is

$$
\hat{\sigma}_{i}^{(k)}=\bar{Q}_{i j}^{(k)}\left(\hat{\epsilon}_{j}^{(k)}-\alpha_{j}^{(\mathrm{k})} \Delta T\right)
$$

Where $\alpha_{j}^{(k)}$ is the CTE of lamina (k),

$$
\hat{\sigma}^{(k)}=\left(\begin{array}{c}
\widehat{\sigma}_{1}^{(\mathrm{k})} \\
\widehat{\sigma}_{2}^{(\mathrm{k})} \\
\hat{\tau}_{12}^{(\mathrm{k})}
\end{array}\right)
$$

and 


$$
\hat{\epsilon}^{(k)}=\left(\begin{array}{c}
\hat{\mathrm{u}}_{, 1}^{(\mathrm{k})} \\
\hat{\mathrm{v}}_{, 2}^{(\mathrm{k})} \\
\hat{\mathrm{u}}_{, 2}^{(\mathrm{k})}+\hat{\mathrm{v}}_{, 1}^{(\mathrm{k})}
\end{array}\right)
$$

Overline denotes undamaged quantities and, $1 ;, 2$ represents partial derivatives as usual.

The constitutive equations for the remaining laminas $(m \neq k)$ can be calculated by using (2.3) with the reduced stiffness matrix, $Q_{i j}^{(m)}$, written in terms of their previously calculated damage values $D_{2}^{(m)}$, $D_{6}^{(m)}$, defined later in 2.1.7, and rotated to the k coordinate system using the usual transformation [2]

$$
Q^{(m)}=[T(\theta)]^{-1}\left(\begin{array}{ccc}
\overline{\mathrm{Q}}_{11}^{(m)} & \sqrt{1-D_{22}^{(m)}} \overline{\mathrm{Q}}_{12}^{(m)} & 0 \\
\sqrt{1-D_{22}^{(m)}} \overline{\mathrm{Q}}_{12}^{(m)} & \left(1-D_{22}^{(m)}\right) \overline{\mathrm{Q}}_{22}^{(m)} & 0 \\
0 & 0 & \left(1-D_{66}^{(m)}\right) \overline{\mathrm{Q}}_{66}^{(m)}
\end{array}\right)[T(\theta)]^{-T}
$$

The damage values belong to a diagonal second order damage tensor defined in [3].

\subsubsection{Shear Lag Equations in Matrix Form}

The functional form of the intralaminar shear stresses is assumed to be:

and

$$
\tau_{13}^{(i)}\left(x_{3}\right)=\tau_{13}^{i-1, i}+\left(\tau_{13}^{i, i+1}-\tau_{13}^{i-1, i}\right) \frac{x_{3}-x_{3}^{i-1, i}}{h^{(i)}}
$$

$$
\tau_{23}^{(i)}\left(x_{3}\right)=\tau_{23}^{i-1, i}+\left(\tau_{23}^{i, i+1}-\tau_{23}^{i-1, i}\right) \frac{x_{3}-x_{3}^{i-1, i}}{h^{(i)}}
$$

That is a linear variation in the $x_{3}$ direction (see Figure 2.3). Where $\tau_{13}^{i-1, i}$ is the shear stress at the interface between the $\mathrm{i}-1$ th and the ith lamina, and $x_{3}^{i-1, i}$ is the position of the interface between the $\mathrm{i}$ 1 th and ith lamina. This assumption is common to several other analytical models and is called the Shear Lag assumption [4].

The shear lag equations are obtained from the constitutive equations for out-of-plane shear strains and stresses by means of weighted averages:

$$
\begin{aligned}
\left(\begin{array}{c}
\hat{\mathrm{u}}^{(i)}-\hat{\mathrm{u}}^{(i-1)} \\
\hat{\mathrm{v}}^{(i)}-\hat{\mathrm{v}}^{(i-1)}
\end{array}\right) & =\frac{h^{(i-1)}}{6}\left(\begin{array}{ll}
S_{45} & S_{55} \\
S_{44} & S_{45}
\end{array}\right)^{(i-1)}\left(\begin{array}{c}
\tau_{23}^{i-2, i-1} \\
\tau_{13}^{i-2, i-1}
\end{array}\right) \\
& +\left(\frac{h^{(i-1)}}{3}\left(\begin{array}{ll}
S_{45} & S_{55} \\
S_{44} & S_{45}
\end{array}\right)^{(i-1)}+\frac{h^{(i)}}{3}\left(\begin{array}{ll}
S_{45} & S_{55} \\
S_{44} & S_{45}
\end{array}\right)^{(i)}\right)\left(\begin{array}{c}
\tau_{23}^{i-1, i} \\
\tau_{13}^{i-1, i}
\end{array}\right) \\
& +\frac{h^{(i)}}{6}\left(\begin{array}{ll}
S_{45} & S_{55} \\
S_{44} & S_{45}
\end{array}\right)^{(i)}\left(\begin{array}{c}
\tau_{23}^{i, i+1} \\
\tau_{13}^{i, i+1}
\end{array}\right)
\end{aligned}
$$


From which the intralaminar shear stresses are obtained as:

$$
\left\{\begin{array}{c}
\tau_{23}^{i, i+1}-\tau_{23}^{i-1, i}=\sum_{j=1}^{n-1}\left\{[H]^{-1}{ }_{2 i-1,2 j-1}-[H]^{-1}{ }_{2 i-3,2 j-1}\right\}\left(\hat{\mathrm{u}}^{(j+1)}-\widehat{\mathrm{u}}^{(j)}\right)+\left\{[H]^{-1}{ }_{2 i-1,2 j}-[H]^{-1}{ }_{2 i-3,2 j}\left(\hat{\mathrm{v}}^{(j+1)}-\hat{\mathrm{v}}^{(j)}\right)\right. \\
\tau_{13}^{i, i+1}-\tau_{13}^{i-1, i}=\sum_{j=1}^{n-1}\left\{[H]^{-1}{ }_{2 i, 2 j-1}-[H]^{-1}{ }_{2 i-2,2 j-1}\right\}\left(\hat{\mathrm{u}}^{(j+1)}-\hat{\mathrm{u}}^{(j)}\right)+\left\{[H]^{-1}{ }_{2 i, 2 j}-[H]^{-1}{ }_{2 i-2,2 j}\left(\hat{\mathrm{v}}^{(j+1)}-\hat{\mathrm{v}}^{(j)}\right)\right\}
\end{array}\right.
$$

Where $\mathrm{H}$ is the matrix that results from the assemblage of (2.9) into a single matrix equation.

These relationships are then used in the equilibrium equations (2.11) and (2.12) to substitute $u$ and $v$ for $\hat{\tau}_{13}$ and $\hat{\tau}_{23}$.

\subsubsection{Solutions of the Equilibrium Equation}

The equilibrium equations for each lamina can be stated as follows

$$
\begin{aligned}
& \widehat{\sigma}_{1,1}^{(i)}+\hat{\tau}_{12,2}^{(i)}+\left(\hat{\tau}_{13}^{i, i+1}-\hat{\tau}_{13}^{i-1, i}\right) / h^{(i)}=0 \\
& \hat{\tau}_{12,1}^{(i)}+\hat{\sigma}_{2,2}^{(i)}+\left(\hat{\tau}_{23}^{i, i+1}-\hat{\tau}_{23}^{i-1, i}\right) / h^{(i)}=0
\end{aligned}
$$

Three solutions are proposed for (2.11) and (2.12)

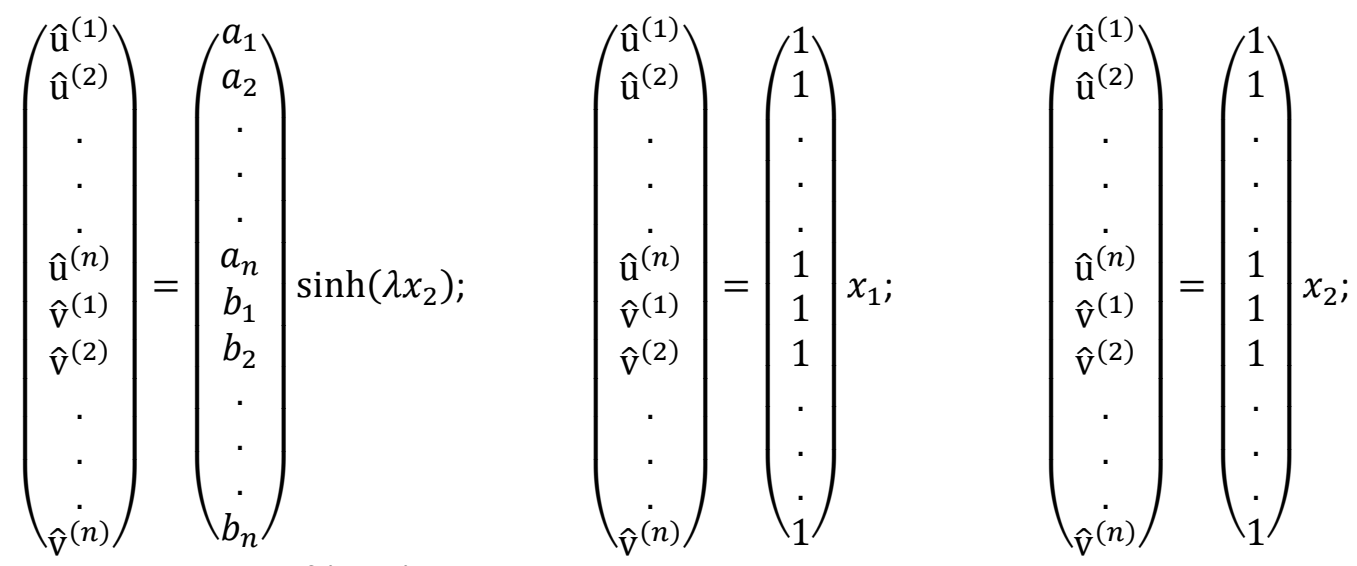

A linear combination of (2.13) yields the general solution

$$
\left(\begin{array}{c}
\hat{\mathrm{u}}^{(1)} \\
\hat{\mathrm{u}}^{(2)} \\
\cdot \\
\cdot \\
\hat{\mathrm{u}}^{(n)} \\
\hat{\mathrm{v}}^{(1)} \\
\hat{\mathrm{v}}^{(2)} \\
\cdot \\
\cdot \\
\cdot \\
\hat{\mathrm{v}}^{(n)}
\end{array}\right)=\sum_{e=1}^{2 N} A_{e}\left(\begin{array}{c}
a_{1} \\
a_{2} \\
\cdot \\
\cdot \\
\cdot \\
a_{n} \\
b_{1} \\
b_{2} \\
\cdot \\
\cdot \\
b_{n}
\end{array}\right)_{e} \sinh \left(\lambda_{e} x_{2}\right)+\left(\begin{array}{c}
a \\
a \\
\cdot \\
\cdot \\
\cdot \\
a \\
b \\
b \\
\cdot \\
\cdot \\
b
\end{array}\right) x_{1}+\left(\begin{array}{c}
b \\
b \\
\cdot \\
\cdot \\
\cdot \\
b \\
a^{*} \\
a^{*} \\
\cdot \\
\cdot \\
\cdot \\
a^{*}
\end{array}\right) x_{2}
$$


Where $A_{e}, a, b$ and $a *$ in the general solution are the coefficients that need to be found to generate the particular solution for each set of boundary conditions [5].

The next step is to evaluate each term in (2.11) and (2.12) using (2.14). This leads to the eigenvalue problem (2.15).

\subsubsection{Eigen system}

When (2.14) is plugged in (2.11) and (2.12), an Eigen system is formed, with eigenvalues $\lambda_{\mathrm{e}}$ and eigenvectors $\left\{\begin{array}{l}a \\ b\end{array}\right\}_{e}$.

$$
\left(\begin{array}{ll}
\alpha_{1} & \beta_{1} \\
\alpha_{2} & \beta_{2}
\end{array}\right)\left\{\begin{array}{l}
a \\
b
\end{array}\right\}+\lambda^{2}\left(\begin{array}{ll}
\zeta_{26} & \zeta_{22} \\
\zeta_{66} & \zeta_{26}
\end{array}\right)\left\{\begin{array}{l}
a \\
b
\end{array}\right\}=\left\{\begin{array}{l}
0 \\
0
\end{array}\right\}
$$

This system yields $2 \mathrm{~N}$ eigenvalues and $2 \mathrm{~N}$ eigenvectors. The $2 \mathrm{~N}-2$ non trivial eigenvalues correspond to the hyperbolic sine solutions, while the two trivial eigenvalues correspond to the linear solutions.

\subsubsection{Boundary Conditions for $\Delta \mathrm{T}=0$}

Let's first consider the case of mechanical loads and no thermal loads. To find the values of $A_{e}, a, a^{*}$ and $b$ the following boundary conditions are enforced:

\subsubsection{Stress free at the cracks}

$$
\begin{aligned}
& \int_{-0.5}^{0.5} \hat{\sigma}_{2}^{(k)}\left(x_{1}, l\right) d x_{1}=0 \\
& \int_{-0.5}^{0.5} \hat{\tau}_{12}^{(k)}\left(x_{1}, l\right) d x_{1}=0
\end{aligned}
$$

\subsubsection{External Loads}

In the $x_{1}$-direction (parallel to the cracks), the load is supported by all the laminas.

$$
\frac{1}{2 l} \sum_{i=1}^{N} h^{(i)} \int_{-l}^{l} \hat{\sigma}_{1}^{(i)}\left(0.5, x_{2}\right) d x_{2}=t_{\text {Laminate }} \hat{\sigma}_{1}
$$

Only the un-cracked or homogenized laminas carry the in-plane shear and normal load in $\mathrm{x}_{2}$-direction.

$$
\begin{aligned}
& \sum_{m \neq k} h^{(m)} \int_{-0.5}^{0.5} \hat{\sigma}_{2}^{(m)}\left(x_{1}, l\right) d x_{1}=t_{\text {Laminate }} \hat{\sigma}_{2} \\
& \sum_{m \neq k} h^{(m)} \int_{-0.5}^{0.5} \hat{\tau}_{12}^{(m)}\left(x_{1}, l\right) d x_{1}=t_{\text {Laminate }} \hat{\tau}_{12}
\end{aligned}
$$

\subsubsection{Homogeneous displacements}

For a homogenized symmetric laminate, membrane loads produce a uniform displacement field through the thickness, i.e. all the un-cracked laminas are subjected to the same displacement 


$$
\begin{array}{ll}
\hat{u}^{(m)}\left(x_{1}, l\right)=\hat{u}^{(r)}\left(x_{1}, l\right) & \forall m \neq k \\
\hat{v}^{(m)}\left(x_{1}, l\right)=\hat{v}^{(r)}\left(x_{1}, l\right) & \forall m \neq k
\end{array}
$$

where $r$ is an un-cracked lamina taken as reference (Lamina 1, except when 1 is cracking then Lamina 2 is taken as reference).

\subsubsection{Boundary conditions matrix ensemble}

Equations (2.16) to (2.22)can be arranged in matrix form as follows:

$$
[B C]_{(2 N+1) x(2 N+1)} \times\left[A_{e} a a^{*} b\right]_{(2 N+1) x 1}=[F]_{(2 N+1) x 1}
$$

The solution of (2.23) for the canonical base of the load space, namely

$$
\left(\begin{array}{c}
\widehat{\sigma}_{1} \\
\widehat{\sigma}_{2} \\
\widehat{\tau}_{12}
\end{array}\right)^{(a)}=\left(\begin{array}{l}
1 \\
0 \\
0
\end{array}\right) ; \quad\left(\begin{array}{c}
\widehat{\sigma}_{1} \\
\widehat{\sigma}_{2} \\
\widehat{\sigma}_{12}
\end{array}\right)^{(b)}=\left(\begin{array}{l}
0 \\
1 \\
0
\end{array}\right) ; \quad\left(\begin{array}{c}
\widehat{\sigma}_{1} \\
\widehat{\sigma}_{2} \\
\widehat{\sigma}_{12}
\end{array}\right)^{(c)}=\left(\begin{array}{l}
0 \\
0 \\
1
\end{array}\right) ; \quad \Delta \mathrm{T}=0
$$

yields a separate set of constants $A_{e}, a$, a* and $b$ and a separate set of deformations $\varepsilon_{1}, \varepsilon_{2}, \gamma_{12}$.

\subsubsection{Boundary Conditions for $\Delta \mathrm{T} \neq 0$}

$\Delta T \neq 0$ adds a constant term to the boundary conditions that can be moved to the right hand side, added to $[\mathrm{F}]$, while the $[\mathrm{BC}]$ matrix remains the same.

The solution set for $\Delta T=1$ results in a strain equals to the laminate apparent coefficient of thermal expansion.

\subsubsection{Laminate properties}

The process of homogenization amounts to remove the cracks from the RVE while replacing their effect by a reduction of the stiffness of the homogenized (i.e., degraded) material.

Before homogenization, the point wise, linear, elastic constitutive equation, with $\Delta \mathrm{T}=0$, is

$$
\epsilon_{i}=\tilde{S}_{i j} \sigma_{j} \quad i, j=1,2,6
$$

Taking the average over the volume of the RVE

$$
\bar{\epsilon}_{i}=\tilde{S}_{i j} \bar{\sigma}_{j} \quad i, j=1,2,6
$$

Where the volume average defined as

$$
\bar{\phi}=\frac{1}{V} \int_{V} \phi d V
$$

After homogenization, the constitutive equation is expressed in terms of stress $\sigma_{\mathrm{j}}^{0}$ and strain $\epsilon_{\mathrm{i}}^{0}$ on the boundary of the RVE, and degraded compliance $S$, as

$$
\epsilon_{i}^{0}=S_{i j} \sigma_{j}^{0} \quad i, j=1,2,6
$$

The CDM principle of strain equivalence between the actual and homogenized configurations states that $\bar{\epsilon}=\epsilon^{0}$ (neglecting the deformation in the cavity of the cracks or inclusions). The principle of strain energy equivalence between the actual and homogenized configurations states that $\frac{1}{2} \bar{\epsilon}_{\mathrm{i}} \sigma_{\mathrm{i}}=\frac{1}{2} \epsilon_{\mathrm{i}}^{0} \sigma_{\mathrm{i}}^{0}$. Since $\sigma=0$ inside the cavity, the deformation of the cavity becomes irrelevant for energy equivalence. 
However, strain equivalence leads to a simpler algorithm, and thus it is used in this work. Therefore, using strain equivalence, $(2.28)$ becomes

$$
\bar{\epsilon}_{i}=\tilde{S}_{i j} \sigma_{j}^{0} \quad i, j=1,2,6
$$

Then the compliance matrix of the laminate in the lamina k coordinate system as a function of the crack density is assembled as

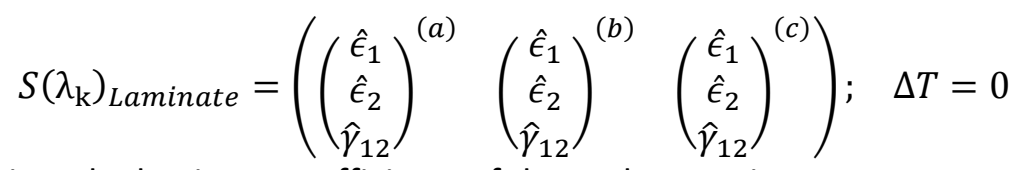

In a similar fashion, the laminate coefficients of thermal expansion are

$$
\left(\begin{array}{c}
\hat{\alpha}_{1} \\
\hat{\alpha}_{2} \\
\hat{\alpha}_{12}
\end{array}\right)\left(\lambda_{\mathrm{k}}\right)_{\text {Laminate }}=\left(\begin{array}{c}
\hat{\epsilon}_{1} \\
\hat{\epsilon}_{2} \\
\hat{\gamma}_{12}
\end{array}\right)^{(d)} ; \Delta T=1
$$

\subsubsection{Lamina properties}

The properties of the cracking lamina can be calculated based on the properties of the laminate

$$
Q^{(k)}\left(\lambda^{(k)}\right)=\left[Q\left(\lambda_{k}\right)_{\text {Laminate }}-\sum_{m \neq k} Q^{(m)} \frac{t^{(m)}}{t_{\text {Laminate }}}\right] \frac{t_{\text {Laminate }}}{t^{(k)}}
$$

Once the lamina properties are known, the damage factors can be calculated

For the thermal expansion coefficients

$$
D_{i j}=1-\frac{Q_{i j}{ }^{(k)}}{\bar{Q}_{i j}{ }^{(k)}}
$$

$$
\alpha^{(k)}=\frac{1}{t^{(k)}} S^{(k)} \cdot\left[t_{\text {Laminate }} Q_{\text {Laminate }} \alpha_{\text {Laminate }}-\sum_{m \neq k} h^{(m)} Q^{(m)} \alpha^{(m)}\right]
$$

where the damage factor is

$$
D_{i}^{\alpha(k)}=1-\frac{\alpha_{i}^{(k)}}{\bar{\alpha}_{i}^{(k)}}
$$

Once the damages for lamina $k$ are known, they are used in the next laminate iteration as the material properties of the lamina, effectively homogenizing the damage and attributing all the loss of stiffness to the stiffness of the cracking lamina.

\subsection{Damage activation function}

The DDM model [1] predicts when the crack density of a lamina should increase by means of a damage activation function $g(\lambda, \epsilon, \Delta T)$, which is essentially the Griffin fracture criteria, namely

$$
\mathrm{G}(\lambda, \epsilon, \Delta \mathrm{T}) \geq \mathrm{G}_{\mathrm{c}}
$$

Where $G$ is the energy release rate (ERR) for the given laminate state $(\lambda, \varepsilon, \Delta T)$ and $G_{c}$ is the critical ERR that is a material property. 
Since intralaminar cracks may propagate in mode I (opening) and mode II (shearing), the ERR needs to be decomposed into $G_{I}$ and $G_{I I}$. This is accomplished by evaluating all the equations in the coordinate system of the cracking lamina k. Then, $G_{I}$ is calculated with $\epsilon=\left\{0, \epsilon_{22}, 0\right\}$ and $G_{I I}$ is calculated with $\epsilon=\left\{0,0, \gamma_{12}\right\}$ [6]. The proposed mode separation is consistent with the method of mechanical work during crack closure in classical fracture mechanics [7], which is the basis for the Virtual Crack Closure Technique (VCCT) broadly adopted in FEA.

The damage activation function may consider or not the interaction between Mode I and Mode II. If the damage activation function does consider interaction, a proposed functional form is [8]

$$
\mathrm{g}(\lambda, \epsilon, \Delta \mathrm{T})^{\text {Interacting }}=(1-r) \sqrt{\frac{G_{I}(\lambda, \epsilon, \Delta \mathrm{T})}{G_{I c}}}+r \frac{G_{I}(\lambda, \epsilon, \Delta \mathrm{T})}{G_{I c}}+\frac{G_{I I}(\lambda, \epsilon, \Delta \mathrm{T})}{G_{I I C}}-1 \leq 0
$$

Where $r=G_{I c} / G_{I I c}$.

If the damage activation function does not consider interaction, then it simply becomes

$$
\mathrm{g}(\lambda, \epsilon, \Delta \mathrm{T})^{\text {Non-interacting }}=\max \left\{\frac{G_{I}(\lambda, \epsilon, \Delta \mathrm{T})}{G_{I c}}, \frac{G_{I I}(\lambda, \epsilon, \Delta \mathrm{T})}{G_{I I c}}\right\}-1 \leq 0
$$

The critical energy release rates $G_{I c}$ and $G_{I I c}$ are not easily found in the literature and have to be fit to experimental data using a methodology that is explained in Chapter 4. Furthermore, the rationale for using one or the other damage activation function will also be explained in Chapter 4.

The energy release rates associated with the introduction of a new crack in the middle of the RVE can be calculated by computing the laminate stiffness and CTE for the current state and for a trial crack density that is double the current crack density. To find the energy associated to those states we use

And

$$
\begin{aligned}
\mathrm{U}_{\mathrm{I}}(\lambda, \epsilon, \Delta \mathrm{T})= & \frac{1}{2} V_{R V E}\left(\epsilon_{2}-\Delta \mathrm{T} \alpha_{2}\right)\left\{Q_{21}(\lambda)\left(\epsilon_{1}-\Delta \mathrm{T} \alpha_{1}\right)+Q_{22}(\lambda)\left(\epsilon_{2}-\Delta \mathrm{T} \alpha_{2}\right)\right. \\
& \left.+Q_{26}(\lambda)\left(\epsilon_{6}-\Delta \mathrm{T} \alpha_{6}\right)\right\}
\end{aligned}
$$

$$
\begin{aligned}
\mathrm{U}_{\mathrm{II}}(\lambda, \epsilon, \Delta \mathrm{T})= & \frac{1}{2} V_{R V E}\left(\epsilon_{6}-\Delta \mathrm{T} \alpha_{6}\right)\left\{Q_{61}(\lambda)\left(\epsilon_{1}-\Delta \mathrm{T} \alpha_{1}\right)+Q_{62}(\lambda)\left(\epsilon_{2}-\Delta \mathrm{T} \alpha_{2}\right)\right. \\
& \left.+Q_{66}(\lambda)\left(\epsilon_{6}-\Delta \mathrm{T}_{6}\right)\right\}
\end{aligned}
$$

Then, to calculate the new area created by the crack

And the ERR for the different modes

$$
\Delta \mathrm{A}=\frac{1}{t_{k}}
$$




$$
\mathrm{G}_{\mathrm{I}}=-\frac{U_{I}(2 \lambda, \epsilon, \Delta \mathrm{T})-U_{I}(\lambda, \epsilon, \Delta \mathrm{T})}{\Delta A}=-\frac{\Delta U_{I}}{\Delta A}
$$

And

$$
\mathrm{G}_{\mathrm{II}}=-\frac{U_{I I}(2 \lambda, \epsilon, \Delta \mathrm{T})-U_{I I}(\lambda, \epsilon, \Delta \mathrm{T})}{\Delta A}=-\frac{\Delta U_{I I}}{\Delta A}
$$

With (2.42) and (2.43), either (2.37) or (2.38) can be evaluated and decide whether or not the crack density should be updated. If the damage activation function predicts damage, a return mapping algorithm (RMA) is then used to propose a crack density change [1]

$$
\Delta \lambda_{\mathrm{k}}=-g_{k} / \frac{\partial g_{k}}{\partial \lambda_{k}}
$$

That is used to re-evaluate the damage activation function until a thermodynamically equilibrium is reached for the laminate. This procedure is done for each value of strain until all the laminas have converged to the right crack density. A flow chart summarizing the procedure is shown in Figure 2.4.

\subsection{References}

[1] EJ Barbero, DH Cortes. A mechanistic model for transverse damage initiation, evolution, and stiffness reduction in laminated composites, Composites Part B: Engineering. 41 (2010) 124-132.

[2] EJ Barbero, Introduction to Composite Materials Design, Second Edition ed., CRC Press, Boca Raton, 2010.

[3] EJ Barbero, Finite element analysis of composite materials, CRC Press, Boca Raton, 2008.

[4] JA Nairn. On the use of shear-lag methods for analysis of stress transfer in unidirectional composites, Mech.Mater. 26 (1997) 63-80.

[5] E Kreyszig, Advanced Engineering Mathematics, 10th ed., Wiley 2011.

[6] J- Rebière, D Gamby. A decomposition of the strain energy release rate associated with the initiation

of transverse cracking, longitudinal cracking and delamination in cross-ply laminates, Composite Structures. 84 (2008) 186-197.

[7] H Tada, PC Paris, G Irwin, The stress analysis of cracks handbook, ASME Press, New York, 2000.

[8] HT Hahn. A Mixed-Mode Fracture Criterion for Composite Materials, Composites technology review. 5 (1983) 26-29. 


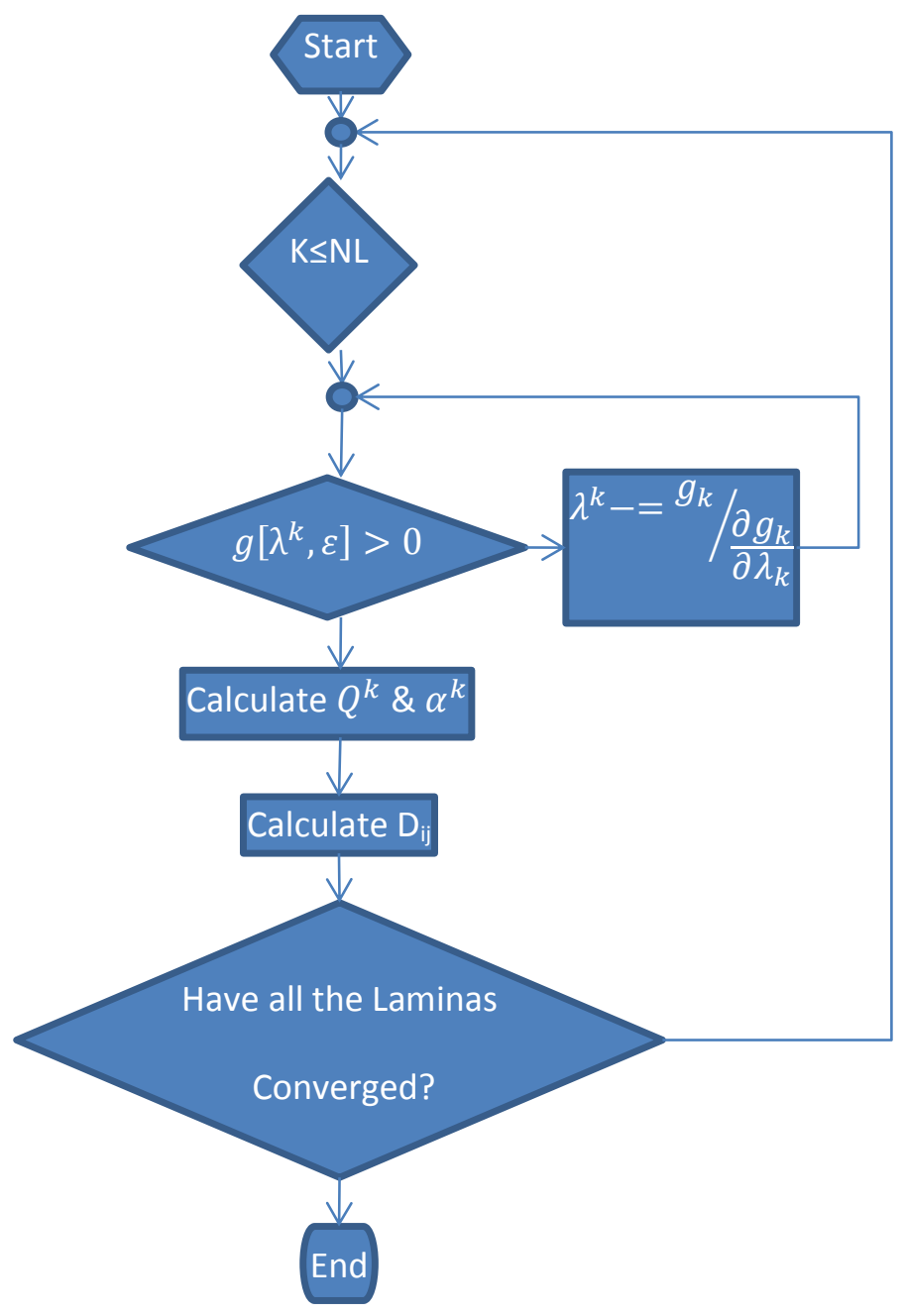

Figure 2.4 Flow chart of DDM for a given value of strain. 


\section{CHAPTER 3. VALIDATION OF THE SHEAR LAG ASSUMPTION}

\subsection{Numerical Homogenization}

In the preceding chapter, the shear lag assumption was used to derive an approximate solution for the elastic displacement field inside the Representative Volume Element (RVE), which is the smallest portion of the material that contains all the peculiarities of the microstructure.

Once the displacement field is known, the reduced stiffness matrix and the apparent coefficients of thermal expansion of the laminate versus crack density can be calculated. The approximate solution needs to be compared to a benchmark solution before trying to predict damage evolution.

A 3D finite element method was chosen as a benchmark because it does not use any of the assumptions of the previous chapter to find the 3D displacement field $u, v, w$.

To calculate the reduced stiffness matrix of the laminate when a lamina $k$ is cracked, the RVE is subjected to an average strain $\bar{\varepsilon}_{\alpha}$ where $\alpha=1,2,4,5,6$ using Voigt contracted notation. With reference to Figures 3.1 and 3.2, the averaged strain is applied by enforcing the following boundary conditions on the displacements:

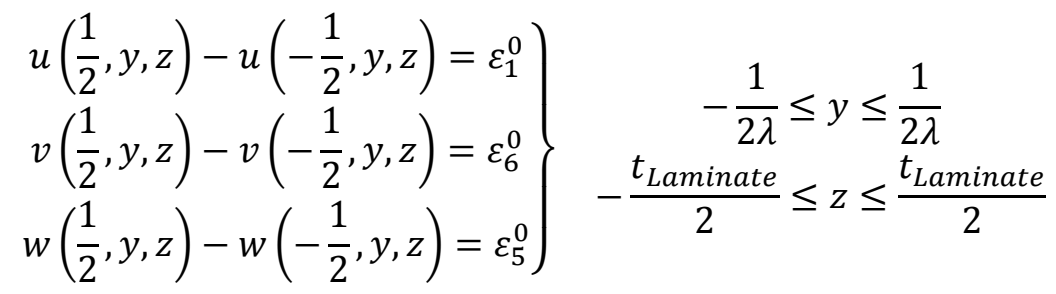

and

$$
\left.\begin{array}{rr}
u\left(x, \frac{1}{2 \lambda}, z\right)-u\left(x,-\frac{1}{2 \lambda}, z\right)=\frac{1}{\lambda} \varepsilon_{6}^{0} \\
v\left(x, \frac{1}{2 \lambda}, z\right)-v\left(x,-\frac{1}{2 \lambda}, z\right)=\frac{1}{\lambda} \varepsilon_{2}^{0} \\
w\left(x, \frac{1}{2 \lambda}, z\right)-w\left(x,-\frac{1}{2 \lambda}, z\right)=\frac{1}{\lambda} \varepsilon_{4}^{0}
\end{array}\right\} \quad-\frac{1}{2} \leq x \leq \frac{1}{2}
$$

Note that $\varepsilon_{\alpha}^{0}$ refer to applied strains, while $\bar{\varepsilon}_{\alpha}$ are averaged strains. The right hand side of the equations is the displacement necessary to enforce the applied strain over the width and height of the RVE respectively.

Equation (3.1) is applied in the faces whose normal is in the x-direction (Vertical faces in Figure 3.2), while (3.2) is applied in the faces whose normal is in the y-direction (Horizontal faces in Figure 3.2). The faces whose normal is in the z-direction do not experience any restriction because they represent the boundary of the laminate and are free to expand or contract. Therefore, $\varepsilon_{3}^{0}$ is not enforced, i.e., the boundary is in a state of plane stress. 


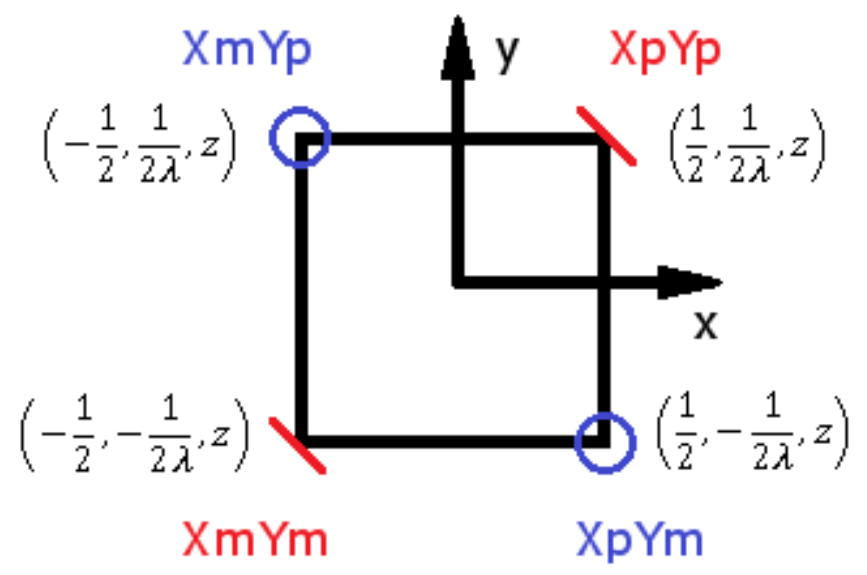

Figure 3.1 Top view of the RVE. Faces are shown as lines and edges are shown as vertices.

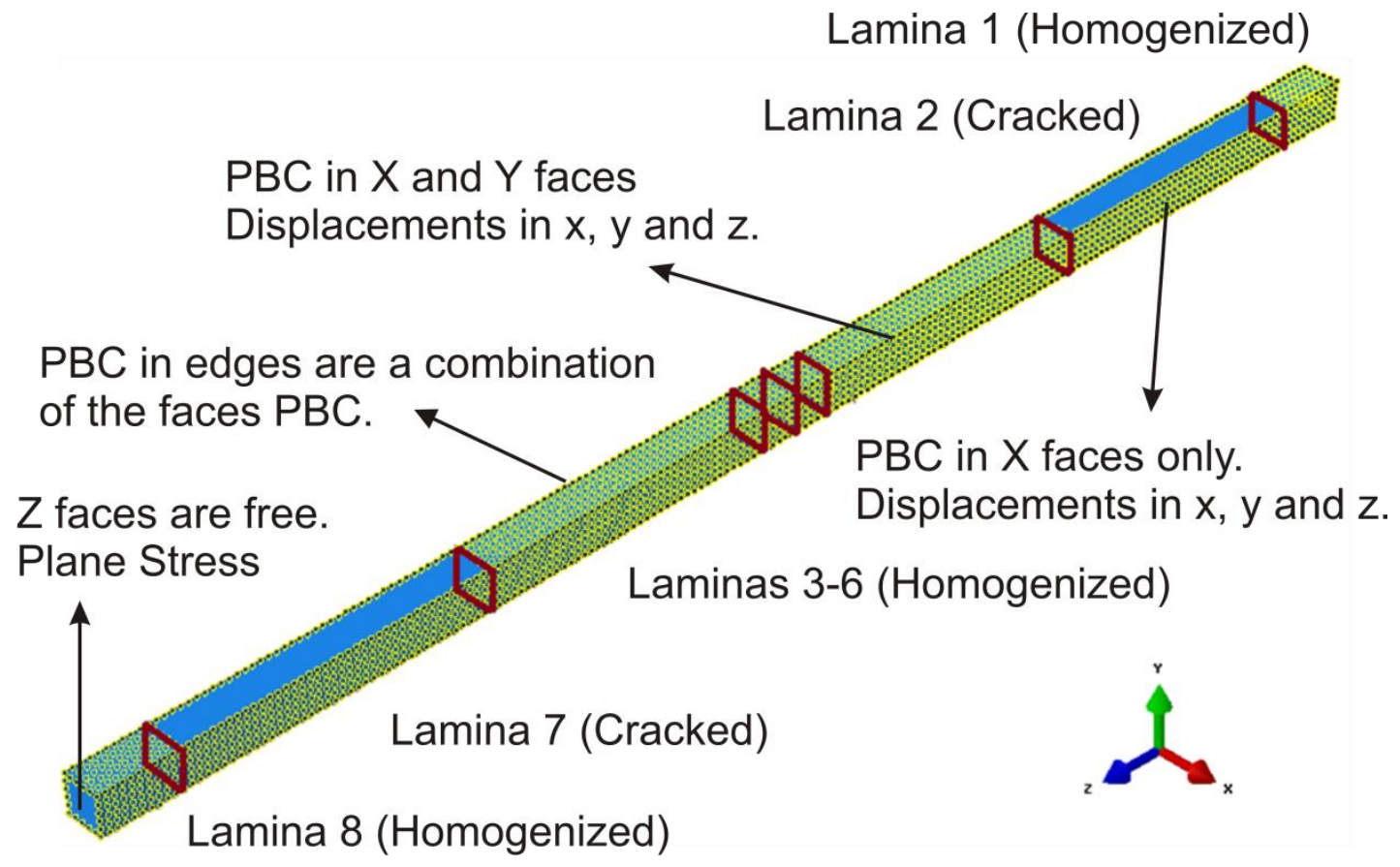

Figure 3.2 Interaction constraints for the case cracking laminas number 2 and 7.

\subsubsection{Edges}

Both (3.1) and (3.2) hold at the edges between horizontal and vertical faces, but cannot be independently enforced because the available degree of freedom in the FE model would be used when the first one is enforced. Therefore, the edges have to be treated separately.

A convenient notation is introduced here for the edges. In each plane that is perpendicular to an axis ( $x$, $y$ and $z$ ) the edges, shown as vertices in Figure 3.1, are named by the faces (lines in Figure 3.1) that intercept. The first face is the face whose normal is in the direction of the axis that follows the axis plane (for plane $x$, face $y$; for plane $y$, face $z$; for plane $z$, face $x$ ). The second face the remaining face. The orientation, either positive (plus, $p$ ) or negative (minus, $m$ ), follows the axis. Four examples are shown in Figure 3.1. 
For edges XpYp (Figure 3.1 top right corner) and XmYp (Figure 3.1 bottom left corner) (3.1) and (3.2) are added together, as follows:

$$
\left.\begin{array}{l}
u\left(\frac{1}{2}, \frac{1}{2 \lambda}, z\right)-u\left(-\frac{1}{2},-\frac{1}{2 \lambda}, z\right)=\varepsilon_{1}^{0}+\frac{1}{\lambda} \varepsilon_{6}^{0} \\
v\left(\frac{1}{2}, \frac{1}{2 \lambda}, z\right)-v\left(-\frac{1}{2},-\frac{1}{2 \lambda}, z\right)=\varepsilon_{6}^{0}+\frac{1}{\lambda} \varepsilon_{2}^{0} \\
w\left(\frac{1}{2}, \frac{1}{2 \lambda}, z\right)-w\left(-\frac{1}{2},-\frac{1}{2 \lambda}, z\right)=\varepsilon_{5}^{0}+\frac{1}{\lambda} \varepsilon_{4}^{0}
\end{array}\right\} \quad-\frac{t_{\text {Laminate }}}{2} \leq z \leq \frac{t_{\text {Laminate }}}{2}
$$

For edges $\mathrm{XmYp}$ and $\mathrm{XpYm},(3.2)$ is subtracted from (3.1), as follows:

$$
\left.\begin{array}{l}
u\left(\frac{1}{2},-\frac{1}{2 \lambda}, z\right)-u\left(-\frac{1}{2}, \frac{1}{2 \lambda}, z\right)=\varepsilon_{1}^{0}-\frac{1}{\lambda} \varepsilon_{6}^{0} \\
v\left(\frac{1}{2},-\frac{1}{2 \lambda}, z\right)-v\left(-\frac{1}{2}, \frac{1}{2 \lambda}, z\right)=\varepsilon_{6}^{0}-\frac{1}{\lambda} \varepsilon_{2}^{0} \\
w\left(\frac{1}{2},-\frac{1}{2 \lambda}, z\right)-w\left(-\frac{1}{2}, \frac{1}{2 \lambda}, z\right)=\varepsilon_{5}^{0}-\frac{1}{\lambda} \varepsilon_{4}^{0}
\end{array}\right\}-\frac{t_{\text {Laminate }}}{2} \leq z \leq \frac{t_{\text {Laminate }}}{2}
$$

\subsubsection{Reduced Stiffness}

The process of homogenization amounts to remove the cracks from the RVE while replacing their effect by a reduction of the stiffness of the homogenized (i.e., degraded) material.

Before homogenization, the point wise, linear, elastic constitutive equation, with $\Delta \mathrm{T}=0$, is

$$
\sigma_{\alpha}=\tilde{Q}_{\text {Laminate }} \epsilon_{\beta} \quad \text { with } \alpha, \beta=1,2,6
$$

Taking the average over the volume of the RVE

$$
\bar{\sigma}_{\alpha}=\tilde{Q}_{\text {Laminate }} \bar{\epsilon}_{\beta} \quad \text { with } \alpha, \beta=1,2,6
$$

After homogeneization, the constitutive equation is expressed in terms of stress $\sigma_{\alpha}^{0}$ and strain $\epsilon_{\alpha}^{0}$ on the boundary of the RVE, and degraded stiffness $Q_{\text {Laminate, as }}$

$$
\sigma_{\alpha}^{0}=Q_{\text {Laminate }} \epsilon_{\beta}^{0} \quad \text { with } \alpha, \beta=1,2,6
$$

The CDM principle of strain equivalence between the actual and homogenized configurations states that $\bar{\epsilon}=\epsilon^{0}$ (neglecting the deformation in the cavity of the cracks or inclusions). Since the material is linearly elastic, this implies that $\bar{\sigma}=\sigma^{0}$.Therefore, in (3.7), we have

$$
\bar{\sigma}_{\alpha}=Q_{\text {Laminate }} \epsilon_{\beta}^{0} \quad \text { with } \alpha, \beta=1,2,6
$$

While the relationship between the averaged intralaminar stress and strain is

$$
\bar{\tau}_{\alpha}=Q_{\text {Laminate }}^{*} \bar{\gamma}_{\beta} \quad \text { with } \alpha, \beta=4,5
$$

Since transverse matrix cracking primarily affects the in-plane properties, (3.9) will essentially remain unchanged and the intact properties can be used, so there is no need to validate the intralaminar stiffness matrix. [1] 
The components of the reduced stiffness matrix $Q_{\text {Laminate }}$ are determined solving three elastic discretized models of the RVE subjected to the periodic boundary conditions, where only one component of the inplane strain $\bar{\epsilon}_{\beta}$ with $\beta=1,2,6$ is different from zero at a time.

By choosing a unit value of applied strain, and once the stress $\sigma_{\alpha}$ is computed by the FEA code, the components of the averaged stress $\bar{\sigma}_{\alpha}$ are calculated by a post processing script, leading to the reduced stiffness matrix defined as: [2]

$$
Q_{\text {Laminate }}=\frac{1}{t_{\text {Laminate }}}\left[\left.\int_{V} \sigma d V\right|_{\epsilon_{1}^{0}=1} ;\left.\quad \int_{V} \sigma d V\right|_{\epsilon_{2}^{0}=1} ;\left.\quad \int_{V} \sigma d V\right|_{\epsilon_{6}^{0}=1}\right]
$$

The integrals are evaluated within each finite element using Gauss quadrature, i.e., transforming the integrals in summations as follows:

$$
\left(Q_{\text {Laminate }}\right)_{\alpha \beta}^{\text {Abaqus }}=\left.\frac{\left.\sum_{i=1}^{\# \text { Gauss Points }} \sigma_{\beta}\right|_{i} V_{i}}{\sum_{i=1}^{\# \text { Gauss Points }} V_{i}} \simeq \frac{1}{t_{\text {Laminate }}} \int_{V} \sigma_{\beta} d V\right|_{\epsilon_{\alpha}^{0}=1}
$$

The coefficients in $Q_{\text {Laminate }}$ are found by setting a different case for each column, as follows.

\subsubsection{First column of $\mathrm{Q}_{\text {Laminate }}$}

In order to determine the components $\left(Q_{\text {Laminate }}\right)_{\alpha 1}$, the following strain is applied to stretch the RVE in the direction parallel to the crack plane (x-direction in Figure ).

$$
\epsilon^{0}=\left\{\begin{array}{l}
1 \\
0 \\
0
\end{array}\right\} \quad \gamma^{0}=\left\{\begin{array}{l}
0 \\
0
\end{array}\right\}
$$

Thus, the displacement boundary conditions for the intact RVE become

and

$$
\left.\begin{array}{c}
u\left(\frac{1}{2}, y, z\right)-u\left(-\frac{1}{2}, y, z\right)=1 \\
v\left(\frac{1}{2}, y, z\right)-v\left(-\frac{1}{2}, y, z\right)=0 \\
w\left(\frac{1}{2}, y, z\right)-w\left(-\frac{1}{2}, y, z\right)=0
\end{array}\right\} \quad-\frac{1}{2 \lambda} \leq y \leq \frac{1}{2 \lambda}
$$

$$
\left.\begin{array}{c}
u\left(x,-\frac{1}{2 \lambda}, z\right)-u\left(x, \frac{1}{2 \lambda}, z\right)=0 \\
v\left(x,-\frac{1}{2 \lambda}, z\right)-v\left(x, \frac{1}{2 \lambda}, z\right)=0 \\
w\left(x,-\frac{1}{2 \lambda}, z\right)-w\left(x, \frac{1}{2 \lambda}, z\right)=0
\end{array}\right\} \quad-\frac{1}{2} \leq x \leq \frac{1}{2}
$$

The first column of $Q_{\text {Laminate }}$ is computed using (3.11) as follows:

$$
\left(Q_{\text {Laminate }}\right)_{\alpha x}=\frac{\left.\sum_{i=1}^{\# \text { Gauss Points }} \sigma_{\beta}\right|_{i} V_{i}}{\sum_{i=1}^{\# \text { Gauss Points }} V_{i}} \quad \text { for } \alpha=1,2,6
$$




\subsubsection{Second column of $Q_{\text {Laminate }}$}

The components $\left(Q_{\text {Laminate }}\right)_{\alpha 2}$ are determined by imposing a unit strain along the direction normal to the crack surface:

$$
\epsilon^{0}=\left\{\begin{array}{l}
0 \\
1 \\
0
\end{array}\right\} \quad \gamma^{0}=\left\{\begin{array}{l}
0 \\
0
\end{array}\right\}
$$

Thus, the displacement boundary conditions for the intact RVE become

$$
\left.\begin{array}{c}
u\left(\frac{1}{2}, y, z\right)-u\left(-\frac{1}{2}, y, z\right)=0 \\
v\left(\frac{1}{2}, y, z\right)-v\left(-\frac{1}{2}, y, z\right)=0 \\
w\left(\frac{1}{2}, y, z\right)-w\left(-\frac{1}{2}, y, z\right)=0
\end{array}\right\} \quad \begin{gathered}
-\frac{1}{2 \lambda} \leq y \leq \frac{1}{2 \lambda} \\
-\frac{t_{\text {Laminate }}}{2} \leq z \leq \frac{t_{\text {Laminate }}}{2}
\end{gathered}
$$

and

$$
\left.\begin{array}{r}
u\left(x,-\frac{1}{2 \lambda}, z\right)-u\left(x, \frac{1}{2 \lambda}, z\right)=0 \\
v\left(x,-\frac{1}{2 \lambda}, z\right)-v\left(x, \frac{1}{2 \lambda}, z\right)=\frac{1}{\lambda} \\
w\left(x,-\frac{1}{2 \lambda}, z\right)-w\left(x, \frac{1}{2 \lambda}, z\right)=0
\end{array}\right\} \quad-\frac{t_{\text {Laminate }}}{2} \leq z \leq \frac{t_{\text {Laminate }}}{2}
$$

Column two of the $Q_{\text {Laminate }}$ is found by using (3.11), as follows

$$
\left(Q_{\text {Laminate }}\right)_{\alpha 2}=\frac{\left.\sum_{i=1}^{\# \text { Gauss Points }} \sigma_{\beta}\right|_{i} V_{i}}{\sum_{i=1}^{\# \text { Gauss Points }} V_{i}} \quad \text { for } \alpha=1,2,6
$$

\subsubsection{Third column of $\mathrm{Q}_{\text {Laminate }}$}

The components $\left(Q_{\text {Laminate }}\right)_{\alpha 6}$ are determined by imposing a unit in-plane shear strain to the laminate:

$$
\epsilon^{0}=\left\{\begin{array}{l}
0 \\
0 \\
1
\end{array}\right\} \quad \gamma^{0}=\left\{\begin{array}{l}
0 \\
0
\end{array}\right\}
$$

Thus, the displacement boundary conditions for the intact RVE become

and

$$
\left.\begin{array}{c}
u\left(\frac{1}{2}, y, z\right)-u\left(-\frac{1}{2}, y, z\right)=0 \\
v\left(\frac{1}{2}, y, z\right)-v\left(-\frac{1}{2}, y, z\right)=0 \\
w\left(\frac{1}{2}, y, z\right)-w\left(-\frac{1}{2}, y, z\right)=1
\end{array}\right\} \quad-\frac{1}{2 \lambda} \leq y \leq \frac{1}{2 \lambda}
$$




$$
\left.\begin{array}{r}
u\left(x,-\frac{1}{2 \lambda}, z\right)-u\left(x, \frac{1}{2 \lambda}, z\right)=0 \\
v\left(x,-\frac{1}{2 \lambda}, z\right)-v\left(x, \frac{1}{2 \lambda}, z\right)=\frac{1}{\lambda} \\
w\left(x,-\frac{1}{2 \lambda}, z\right)-w\left(x, \frac{1}{2 \lambda}, z\right)=0
\end{array}\right\} \quad-\frac{t_{\text {Laminate }}}{2} \leq z \leq \frac{t_{\text {Laminate }}}{2}
$$

The coefficients of column three of the $Q_{\text {Laminate }}$ are found by using (3.11), as follows

$$
\left(Q_{\text {Laminate }}\right)_{\alpha 6}=\frac{\left.\sum_{i=1}^{\# \text { Gauss Points }} \sigma_{\beta}\right|_{i} V_{i}}{\sum_{i=1}^{\# \text { Gauss Points }} V_{i}} \quad \text { for } \alpha=1,2,6
$$

\subsubsection{Laminate apparent CTE}

A similar procedure to the one used for the laminate stiffness can be applied to obtain the laminate apparent CTE. All the components of the applied strain for this case are zero.

$$
\epsilon^{0}=\left\{\begin{array}{l}
0 \\
0 \\
0
\end{array}\right\} \quad \gamma^{0}=\left\{\begin{array}{l}
0 \\
0
\end{array}\right\}
$$

And (3.6) is modified slightly to account for the thermal effects, namely:

$$
\bar{\sigma}_{\alpha}=Q_{\text {Laminate }}\left(\bar{\epsilon}_{\beta}-\bar{\alpha}_{\text {Laminate }} \Delta T\right) \text { with } \alpha, \beta=1,2,6
$$

Imposing $\Delta T=1$, and using the laminte stiffness recently calculated, results,

$$
\bar{\alpha}_{\text {Laminate }}=-\left(Q_{\text {Laminate }}\right)^{-1} \bar{\sigma}_{\alpha} \text { with } \alpha, \beta=1,2,6
$$

\subsection{Implementation}

Since periodic boundary conditions are not readily available in Abaqus, they were implemented in this work. For this, a Python program was developed. The program generates the RVE, meshes, then generates constrains in the faces that are periodic and finally solves the model and stores the averaged stresses. The program was validated against known solutions and experimental data.

First, when no crack is present, the solution must coincide with the Classical Laminate Theory (CLT) solution. Since the strain and stresses in this case are piecewise constant thought the body, no mesh refinement is necessary. Still, different meshes were used to validate that the solution does not change for different number of nodes at the faces and thus the implementation is correct.

Laminate 1 (

Table 3.1) was used for this validation. The in-plane reduced stiffness matrix predicted by CLT, in laminate coordinate system, defined as:

$$
Q_{\alpha \beta}=\frac{1}{t_{\text {Laminate }}} \sum_{k=1}^{N}\left(\bar{Q}_{\alpha \beta}\right) t_{k} \quad \text { with } \alpha, \beta=1,2,6
$$

Was calculated with www.cadec-online.com and resulted in 


$$
Q_{\text {intact }}^{\mathrm{CLT}}=\left(\begin{array}{ccc}
22108.664 & 9062.480 & 0 \\
9062.480 & 26379.612 & 0 \\
0 & 0 & 10993.620
\end{array}\right) M P a
$$

Table 3.1 Laminates analyzed.

\begin{tabular}{|c|c|c|c|}
\hline Laminate & Stacking Sequence & Material & Reference \\
\hline 1 & {$\left[055_{4}-55_{4} 0_{1 / 2}\right] \mathrm{s}$} & Fiberite/HyE 9082Af & [9] \\
\hline 2 & {$\left[\begin{array}{lll}0 & 90_{8} & 0_{1 / 2}\end{array}\right] \mathrm{s}$} & “ & " \\
\hline 3 & {$\left[070_{4}-70_{4} 0_{1 / 2}\right] \mathrm{s}$} & " & " \\
\hline 4 & {$\left[09_{2}\right] \mathrm{s}$} & Avimid $^{\circledR}$ K Polymer/IM6 & [10] \\
\hline 5 & {$\left[\begin{array}{lll}0 & 90_{3}\end{array}\right] \mathrm{s}$} & " & " \\
\hline 6 & {$\left[0_{2} 90_{2}\right] \mathrm{s}$} & " & " \\
\hline 7 & {$\left[0_{2}, 90_{4}\right] \mathrm{s}$} & " & " \\
\hline 8 & {$\left[09_{2}\right] \mathrm{s}$} & Fiberite 934/T300 & " \\
\hline 9 & {$\left[09_{4}\right] \mathrm{s}$} & “ & “ \\
\hline 10 & {$\left[0_{2} 90\right] \mathrm{s}$} & " & " \\
\hline 11 & {$\left[\mathrm{O}_{2} 90_{2}\right] \mathrm{s}$} & " & “ \\
\hline 12 & {$\left[0_{2} 90_{4}\right] \mathrm{s}$} & “ & " \\
\hline 13 & {$\left[09_{2}\right] \mathrm{s}$} & Hercules 3501 -6/AS4 & " \\
\hline 14 & {$\left[0_{2} 90_{2}\right] \mathrm{s}$} & “ & " \\
\hline 15 & {$\left[ \pm 1590_{4}\right] \mathrm{s}$} & Fiberite/HyE 9082Af & [11] \\
\hline 16 & {$\left[ \pm 3090_{4}\right] \mathrm{s}$} & $"$ & " \\
\hline 17 & {$\left[ \pm 4090_{4}\right] \mathrm{s}$} & " & " \\
\hline 18 & {$\left[0_{2}, 90_{4}\right] \mathrm{s}$} & “" & " \\
\hline
\end{tabular}

Table 3.2 Material properties.

\begin{tabular}{lrrrrrrrr} 
& \multicolumn{1}{c}{$\mathbf{E}_{\mathbf{1}}$} & \multicolumn{1}{c}{$\mathbf{E}_{\mathbf{2}}$} & \multicolumn{1}{c}{$\mathbf{G}_{\mathbf{1 2}}$} & \multicolumn{1}{c}{$\mathbf{V}_{\mathbf{1 2}}$} & \multicolumn{1}{c}{$\mathbf{G}_{\mathbf{2 3}}$} & \multicolumn{1}{c}{$\boldsymbol{\alpha}_{\mathbf{1}}$} & \multicolumn{1}{c}{$\boldsymbol{\alpha}_{\mathbf{2}}$} & \multicolumn{1}{c}{$\mathbf{t}_{\mathbf{k}}$} \\
& {$[\mathrm{GPa}]$} & \multicolumn{1}{c}{$[\mathrm{GPa}]$} & \multicolumn{1}{c}{$[\mathrm{GPa}]$} & & {$[\mathrm{GPa}]$} & \multicolumn{1}{c}{$\left[0^{-6} / \mathrm{K}\right]$} & {$\left[10^{-6} / \mathrm{K}\right]$} & {$[\mathrm{mm}]$} \\
\hline Fiberite/HyE 9082Af [9] & 44.7 & 12.7 & 5.8 & 0.297 & 4.5 & 8.42 & 18.4 & 0.144 \\
\hline Avimid $^{\circledR}$ K Polymer/IM6 [10] & 134 & 9.8 & 5.5 & 0.3 & 3.6 & -0.09 & 28.8 & 0.144 \\
\hline Fiberite 934/T300 [10] & 128 & 7.2 & 4 & 0.3 & 2.4 & -0.09 & 28.8 & 0.144 \\
\hline Hercules 3501 -6/AS4 [9] & 130 & 9.7 & 5 & 0.3 & 3.6 & -0.09 & 28.8 & 0.144
\end{tabular}

The reduced stiffness matrix in lamina $k=2$ coordinate system can be calculated rotating the previous matrix, using the rotation matrices. [3]

$$
\bar{Q}_{\text {intact }}^{\mathrm{CLT}}=\left(\begin{array}{ccc}
27979.25 & 6057.74 & -90.29 \\
6057.74 & 26518.51 & 2096.98 \\
-90.29 & 2096.98 & 7988.88
\end{array}\right) M P a
$$

Therefore, the quality of the finite element simulation results can be benchmarked by this result.

\subsubsection{Intact RVE}

The procedure followed to construct the model for the undamaged (intact) RVE is as follows: 
1. Creation of the part:

The dimension in the $x$-direction is the thickness of one lamina instead of a unitary dimension (Compare with (3.1) and (3.2)). This is done to have a good element's aspect ratio. The Python

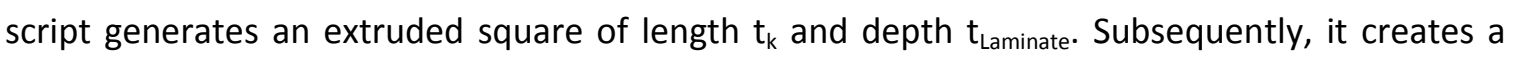
part with a 3D deformable body.

2. Definition of the material:

An elastic material of type Engineering Constants is created and the properties of the material Fiberite/HyE 9082Af (Table 3.2) are assigned to it.

3. Create the section:

A homogenous solid section is created with the previous material.

4. Creation of the sets:

In order to apply the boundary conditions a number of sets are defined, namely, the faces whose normal points either in $x$ or $y$ direction, positive or negative orientation, excluding the edges, and the edges between those faces. A special algorithm was devised to search for these features.

5. Create the Laminate Stacking Sequence:

Planes are created at the interfaces between laminas. A datum plane is generated offsetting the $x y$-plane by the accumulated thickness. Immediately afterwards, the part is partitioned using those planes. The partition process creates a cell for each lamina in the laminate. A material orientation, relative to $k$, is assigned to each cell. The section created in 3 , which is independent of the orientation, is then assigned to every cell.

6. Create the instance:

A new instance is created in the root assembly and translated so the mid-plane is located at coordinate $\mathrm{z}=0$.

7. Create Step:

The Python script creates a new Static General step.

8. Define Field Output request:

The variables required for the analysis are requested from the solver: E (Strain), S (Stress), IVOL (Integration Volume), U (displacements).

9. Create a Reference Point: 
A reference point is created and a unitary constant displacement is assigned to it. This point is necessary because the constraint equations in Abaqus relay on degrees of freedom. Since the periodic boundary conditions (3.1) and (3.2) contain a constant term that represents the applied strain, a degree of freedom is needed to apply such constrain throughout the simulation; this can only be achieved by using a Reference Point.

10. Create the Mesh:

A C3D8 (8-node linear brick) element for each layer is generated by the python script.

11. Restrain Rigid Body Motion:

The node closer to the center of the RVE is fixed in $x, y$ and $z$ direction to prevent rigid body motion.

Once the RVE is constructed, the model is used as a template to generate each of the three cases, as follows:

1. The template model is copied to new models named Case-a, Case-b, and Case-c

2. Create Periodic Boundary Conditions:

a. Opposite faces constraints

i. X axis

The face whose normal is in the direction of $x$ positive is chosen as the master, while the face whose normal is in the negative direction is slave.

The nodes in the slave face are searched to pair with the nodes in the master face. This is done by looping through the array of nodes in the master face and selecting the slave node with the minimum distance in the yz plane.

In the interaction module, a constraint equation such as (3.1) or (3.2) is created for each direction $(x, y$ and $z$ ). This equation relates the displacement of the master and the slave node with the displacement that results from the imposed strain field multiplied by the reference node unitary displacement.

ii. $Y$ axis

The same procedure used for the $\mathrm{x}$ axis is repeated for the $\mathrm{y}$ axis.

b. Edges constraints

As it was said before, the constraint equations in the nodes at the edges are the combination of the equations at the faces.

The procedure followed to pick the master and slave edges is as follows: 
In the xy plane (Figure 3.1) there are four edges and the equations have to relate two pairs: $\mathrm{XmYm}$ with $\mathrm{XpYp}$ and $\mathrm{XmYp}$ with $\mathrm{XpYm}$. If we think of $m$ as 0 and $p$ as 1 , then the pairs can be generated with a loop with $i=0,1$ (the two pairs) and the master is the loop index value i while the slave is the bitwise negation (word size is two bits).

A truth table for this relation is shown in Table 3.3.

This procedure automates the selection of pairs of edges in the Python script.

Once the master and slave edges have been defined, a pair algorithm is used to find the slave node for each master node. The algorithm looks for the pair with the minimum distance in the $z$ direction.

A constraint equation ((3.3) for XmYm-XpYp and (3.4) for XmYp-XpYm) is added to the model for each pair of master-slave nodes, relating the displacement in $x$, and $y$ direction with the displacement that results from the imposed strain field.

3. Apply predefined field

If the step involves a change of temperature, a predefined field is assigned to the whole laminate where the temperature is zero for the initial step and $\Delta T$ for the following step.

4. Create job

A job is created and submitted. The number of physical processors is assigned to execute the job in parallel.

5. Get the Laminate Reduced Stiffness Matrix column (or CTE)

For each case $a, b, c$, the field output corresponding to the stresses in the $k_{0}$ coordinate system (Global Coordinate system for the model) is looped over the integration points and an accumulation variable is incremented by the stress value times the integration volume. The same procedure is followed for the strain.

At the end of the loop, the total stresses and strains are divided by the total volume, yielding the average strains and stresses in the laminate. The three components of stress correspond to the column 1, 2, and 3 in the reduced stiffness matrix of the laminate for the strain cases or to the laminate apparent CTE for the temperature change case.

6. Get the lamina-k Reduced Stiffness Matrix column (or CTE)

A similar procedure is used here over the lamina $\mathrm{k}$ cell instead of the whole laminate. 
Table 3.3 Truth table for edges in the RVE.

\begin{tabular}{lllll}
\hline $\mathbf{i}$ & Binary & Binary & Master & Slave \\
\hline $\mathbf{0}$ & 00 or $\mathrm{mm}$ & 11 or $\mathrm{pp}$ & $\mathrm{XmYm}$ & $\mathrm{XpYp}$ \\
1 & 01 or $\mathrm{mp}$ & 10 or $\mathrm{pm}$ & $\mathrm{XmYp}$ & $\mathrm{XpYm}$ \\
\hline
\end{tabular}

The reduced stiffness matrix for laminate 1 using eight C3D8 elements and thirty-six nodes is

$$
\bar{Q}_{\text {intact }}^{\text {FEA }}=\left(\begin{array}{ccc}
27979.25 & 6057.74 & -90.29 \\
6057.74 & 26518.51 & 2096.98 \\
-90.29 & 2096.98 & 7988.88
\end{array}\right) M P a
$$

The number of significant digits (those digits in a number that carry meaning contributing to its precision) in the previous matrix can be assessed by employing the following equation in each term:

$$
\text { \# Significant digits }{ }_{\alpha \beta}=-\log _{10} \frac{\left|\bar{Q}_{\alpha \beta}^{\mathrm{CLT}}-\bar{Q}_{\alpha \beta}^{\mathrm{FEA}}\right|}{\bar{Q}_{\alpha \beta}^{\mathrm{CLT}}} \quad \text { with } \alpha, \beta=1,2,6
$$

In matrix form, the results are

$$
\text { \# Significant digits }=\left(\begin{array}{lll}
7 & 7 & 6 \\
7 & 7 & 8 \\
5 & 6 & 7
\end{array}\right)
$$

Equation (3.30) coincides with (3.29) up to single precision, since Abaqus results are written in single precision, we conclude that no error is found between the Abaqus solution and the CLT solution.

The homogenized (averaged) strains for each case are shown in Table 3.4.

Note in Table 3.4 that all the zeros are zeros up to single precision accuracy and ones are ones up to single precision accuracy. The Strain component 3 is not zero, confirming the plane stress state.

Table 3.4 Laminate 1 averaged strains in the intact RVE for the three boundary condition cases.

\begin{tabular}{r|rrr}
\hline & \multicolumn{1}{c}{ Case (a) } & \multicolumn{1}{c}{ Case (b) } & \multicolumn{1}{c}{ Case (c) } \\
\hline $\boldsymbol{\varepsilon}_{1}$ & $1+4 \mathrm{E}-08$ & $-8 \mathrm{E}-09$ & $-1 \mathrm{E}-08$ \\
$\boldsymbol{\varepsilon}_{2}$ & $-8 \mathrm{E}-09$ & $1+4 \mathrm{E}-08$ & $1 \mathrm{E}-08$ \\
$\boldsymbol{\varepsilon}_{3}$ & -0.43 & -0.43 & 0.00058 \\
$\boldsymbol{\varepsilon}_{6}$ & $4 \mathrm{E}-08$ & $-6 \mathrm{E}-09$ & $1+4 \mathrm{E}-08$ \\
$\boldsymbol{\varepsilon}_{5}$ & $-2 \mathrm{E}-16$ & $-8 \mathrm{E}-16$ & $7 \mathrm{E}-16$ \\
$\boldsymbol{\varepsilon}_{4}$ & $-9 \mathrm{E}-15$ & $-1 \mathrm{E}-14$ & $6 \mathrm{E}-15$ \\
\hline
\end{tabular}

\subsubsection{Damaged RVE}

Once the procedure for the construction of the intact RVE has been validated, the damaged RVE was simulated. In this section, the differences with the intact template model are explained. 
1. Creation of the Part:

The RVE size in the $y$-direction is the inverse of the crack density because the cracks are assumed to be equally spaced along that direction by a distance $1 / \lambda$. The RVE size in the $x-$ direction is the minimum between the inverse of the crack density and the lamina thickness rather than one (Compare with (3.1) and (3.2)) because this choice leads to elements with a good aspect ratio and reduces the number of elements.

2. Nondimensionalization:

The lengths, elastic moduli, and coefficients of thermal expansion are nondimensionalized dividing by the maximum of each type. This is done to minimize the numerical errors. The scale factors are saved for subsequent use to dimensionalize back the results.

3. Sub laminates partition:

Because the crack faces are normal to the y-axis, the periodicity in the faces normal to the $y$ axis is broken at the cracking laminas. Therefore, the part is divided in five cells so each cell can be treated separately:

1. Homogenized laminas below lamina $\mathrm{k}$ (Lamina number 1 in Figure 3.2)

2. Cracked lamina $\mathrm{k}_{0}$ (Lamina number 2 in Figure 3.2)

3. Homogenized laminas between $k$ and symmetric cracked lamina $\left(N L+1-k_{0}\right)$, with $N L$ being half the number of laminas in the laminate (Laminas number 3 to 6 in Figure 3.2).

4. Cracked lamina NL+1-k (Lamina number 7 in Figure 3.2)

5. Homogenized laminas above the NL+1-k lamina (Lamina number 8 in Figure 3.2)

4. Creation of the sets:

1. In the homogenized cells ( 1,3 and 5$)$, constraints equations are set in pairs for the four faces whose normal is in the $X$ and $Y$, plus and minus orientation. This is done in the same fashion as in the intact case. The sets exclude the edges between the $X$ and $Y$ faces. Those edges feature constraint equations that are the combination of the periodic boundary conditions for faces $X$ and $Y$ as explained in section 3.1.1.

2. In the cracked lamina cells ( 2 and 4 ), constraint equations are set only for the faces whose normal is in $x$-direction, plus and minus direction. The sets exclude the edges between the $X$ and $Z$ faces because these nodes were included in the homogenized cells sets. The cracks faces (normal in y-direction) are left unconstrained because they are stress free surfaces $\left(\sigma_{2}=0\right)$.

5. Creation of the Laminate Stacking Sequence:

Cells 1, 3, and 5 may contain several laminas, therefore these cells (or sub laminates) are partitioned further into laminas, and a similar procedure described for the intact case is followed for the assignment of the material orientation and section.

6. Create the mesh: 
The initial seed size is calculated based on the aspect ratio of the RVE, and then it is modified until the number of elements in the mesh is in the desired range that ensures accuracy and affordable computational time.

Similarly to the intact case, once the template model has been defined, each strain case is executed and the resultant homogenized stresses stored. The difference between the intact and cracking models are highlighted below.

1. Create Periodic Boundary Conditions in Homogenized Cells:

a) Faces constraints

For each homogenized sub laminte $(1,3$, and 5$)$ and for each normal axis ( $x$ and $y)$ the nodes are paired following the procedure explained in the intact model. Afterwards, constrains equations in $x, y$ and $z$ are created between the nodes in the minus a plus orientation.

b) Vertical edges constraints

The same procedure to find the pair master-slave edges outlined in the intact model is followed for the xy edges in the homogenized cells.

2. Create Periodic Boundary Conditions in cracked $\mathrm{k}_{0}$ and NL+1-k cells:

a) $X$ Faces constraints

For each cracking lamina (cells 2 and 4) the nodes in the faces whose normal is in the $x$ direction are paired following the procedure explained in the intact model. Afterwards, constrains equations in $x, y$, and $z$ are created between the nodes in the plus ( $p$ ) or minus (m) orientation. The crack surfaces are left unconstrained.

\subsubsection{Parametric study}

In order to calculate the laminate and lamina properties for different values of crack density in a wide range (0.01 to 1000), a uniform distribution of random numbers was used. The same program was replicated in up to twenty computers that would generate a random value of crack density according to the formula

$$
\lambda=10^{U(-2,3)}
$$

Upon a successful calculation, the Python script uploaded the information to a Microsoft SQL Server 2008 database, this approach prevented concurrency issues and facilitated enormously the task of gathering the information and accelerated the simulation time up to 20 times.

\subsection{Results}

A mesh convergence study is presented for the case when the crack density is equal to the inverse of the lamina thickness $\left(\lambda=1 / t_{k}\right)$ for Laminate 1 . The percent error is calculated as

$$
\epsilon=\frac{\left|Q_{11}(n)-Q_{11}(N)\right|}{Q_{11}(N)} 100 \%
$$


Where $\mathrm{n}, \mathrm{N}$ are the number of nodes and the maximum number of nodes, respectively as show in Figure 3.3, $\mathrm{N}=3390$ for the C3D8 element and , $\mathrm{N}=1000$ for the C3D20 element.

As can be seen in Figure 3.3, the maximum error is less that $0.20 \%$.

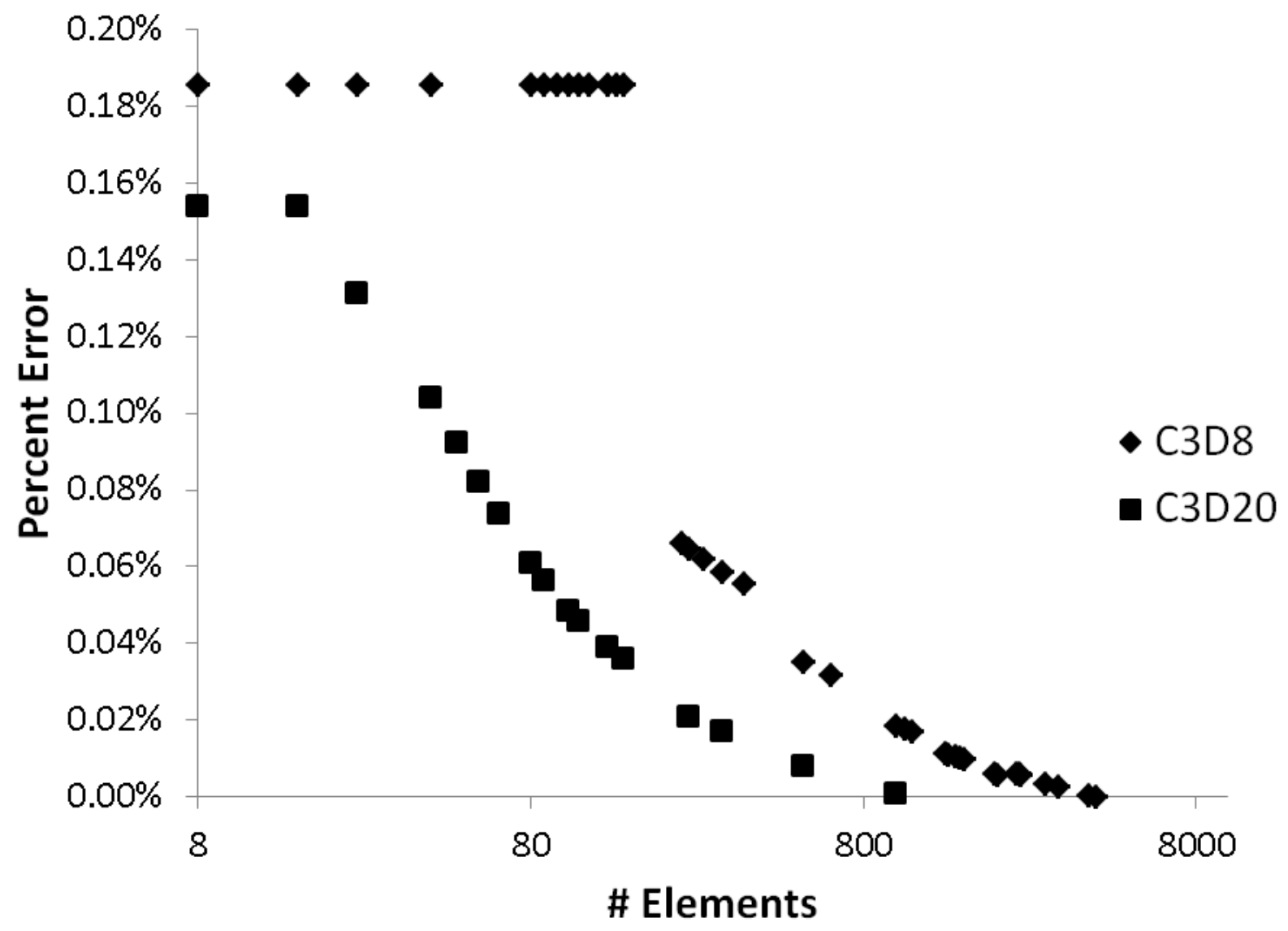

Figure 3.3 Percent error vs. \# Elements for C3D8 and C3D20 element types.

A strain contour plot is shown in Figure 3.4, where the RVE is replicated eight times to aid the visualization. The strain field in the homogenized laminas matches the applied strain, while cracked laminas experience less deformation. In the proximity of the crack tip the strain increases up to $30 \%$ from the applied strain $\left(\epsilon_{22}=1.3\right)$. 


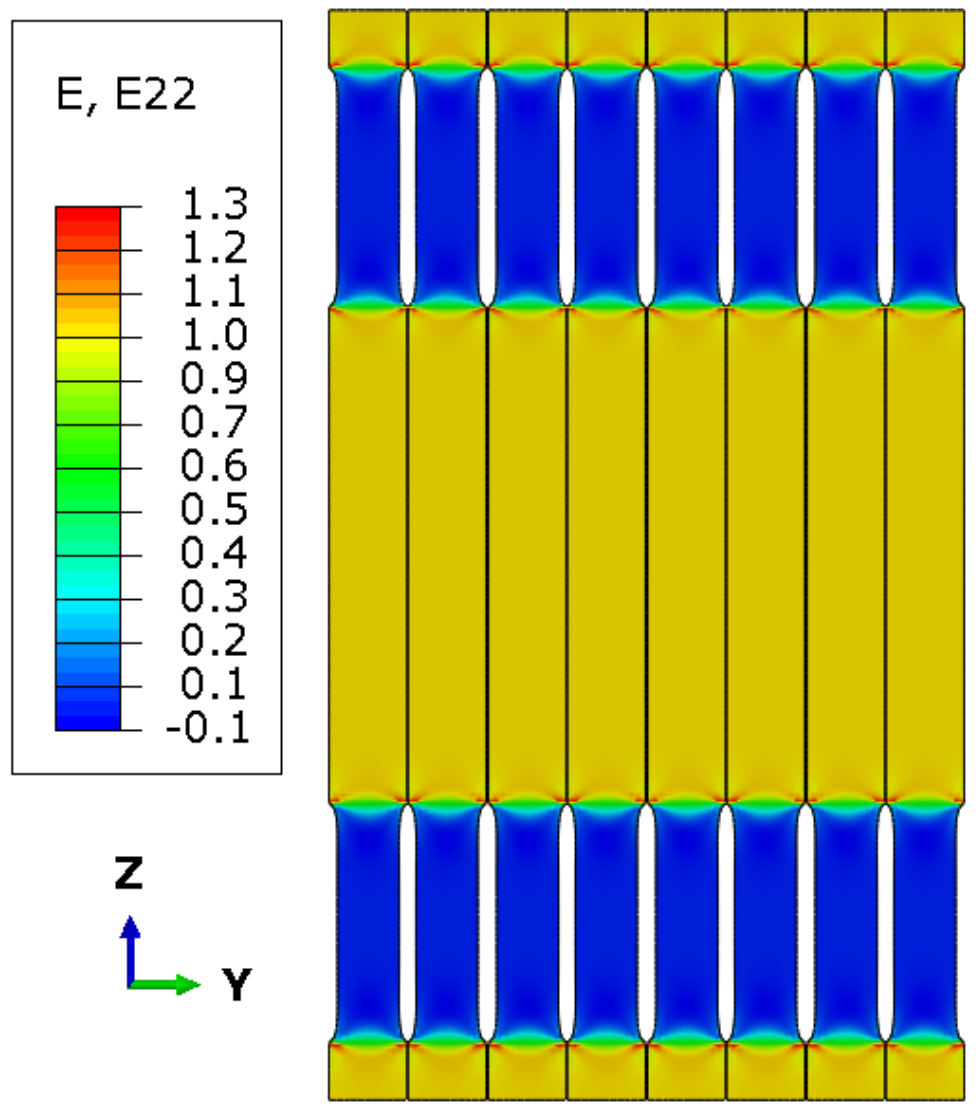

Figure 3.4 Strain field for eight consecutive RVEs for $\varepsilon_{2}^{0}=1$.

Calculated longitudinal laminate modulus $\left(E_{x}\right)$ vs. crack densities for laminates number 1 and 6 are shown in Figures 3.5 and 3.6. Results for the remaining laminates listed in

Table 3.1 are shown in the Appendix.

The asymptotic values of $E_{x}$ for very low $\left(10^{-2}\right)$ and very high $\left(10^{3}\right)$ crack density coincide with the CLT solution obtained assuming that the cracked laminas are intact and fully damaged, respectively. The FEA solution is the best solution available in the sense that it is obtained with the minimum set of assumptions and simplifications. That is, a 3D elasticity problem of a periodically cracked media is discretized with a mesh that has been shown to yield negligible discretization error (Figure 3.3). The periodicity conditions are applied exactly as far as the discretization used allows it. No other approximations are introduced. In this sense, the DDM solution is compared to the FEM solution, with the latter considered to be the benchmark solution. Note that DDM assumes linear distribution of intralaminar shear stress $\sigma_{4}$ and $\sigma_{5}$ in each lamina, while the FEM solution does not.

Transverse laminate modulus $E_{y}$ vs. crack density plots are shown in Figures 3.7 and 3.8. Plots for the remaining laminates are shown in the Appendix. Again, the CLT solution validates the asymptotes and the FEM solution becomes the benchmark in between. 
Apparent laminate coefficient of thermal expansion (CTE) in both $x$ - and y-directions vs. crack density are shown in Figures 3.9 and 3.10 for laminates number 1 and 6, respectively. The lamina CTE remains constant for the six laminates analyzed, and therefore the damage variables are zero.

Experimental results are compared to the FEA solution in Figures 3.11 and 3.12 for laminate 2, displaying good agreement.

All the comparisons, including those reported in the Appendix, are good, so is the comparison of DDM to FEM. The most notable difference occurs in the Poisson's ratio versus crack density shown in Figure 3.13 for laminate 3 , in the vicinity of crack density equals seven lamina thicknesses.

The laminate modulus reduction can be homogenized by interpreting it as a modulus reduction of the cracked lamina. Such reduction can be in turn be expressed in terms of ply damage variables $D_{11}^{i}, D_{22}^{i}$, and $D_{66}^{i}$ defined as:

$$
D_{\alpha \beta}^{i}=1-\frac{\left(Q_{\alpha \beta}^{\text {Damaged }}\right)^{i}}{\left(Q_{\alpha \beta}^{\text {Intact }}\right)^{i}} \text { with } \alpha=\beta=1,2,6
$$

Where $\left(Q_{\alpha \beta}^{\text {Damaged }}\right)^{i},\left(Q_{\alpha \beta}^{\text {Intact }}\right)^{i}$, are the damaged and undamaged lamina I reduced stiffness matrix in lamina coordinate system, respectively.

The FEM calculated damage variables for laminate 1 and 6 are shown in Figures 3.14-17. Again, the DDM solution is close to the FEM benchmark. Note in Figures 3.14-17 that $D_{12}=D_{22}$ for all values of crack densities. Therefore,

$$
\frac{Q_{12}}{Q_{22}}=v_{12}=\text { constant }
$$

Since $E_{1} \approx$ constant, then $v_{21} / E_{2}=$ constant, which agrees with the hypothesis of Nuismer and Tan $[4,5]$ that is used in several damage models such as [6-8].

Note also that the ratio $D_{66} / D_{22}$ is not constant (see Figures 3.18 and 3.19), but is close to one.

\subsection{Conclusions}

The FEM benchmark solution is validated by the CLT solution for asymptotic values of crack density. Also, experimental data compares well with the FEM solution. The comparison between the approximate DDM solution and FEM solution reveals that the shortcomings introduced by the approximations in DDM are not severe. Notable is the fact that $D_{12}=D_{22}$ and $D_{66} / D_{22} \approx 1$ in all of the six laminates reported, which include both Glass/Epoxy and Carbon/Epoxy laminates.

\subsection{Acknowledgment}

The author of this thesis wish to thank Mr. Rene Sprunger from 3DS for facilitating a 3DS's teaching grant that allowed me to learn Abaqus/CA. Special thanks to Dr. Fritz Campos for the first version of the homogenization algorithm. 


\subsection{References}

[1] E Adolfsson, P Gudmundson. Thermoelastic properties in combined bending and extension of thin composite laminates with transverse matrix cracks, Int.J.Solids Structures. 34 (1997) 2035-2060.

[2] EJ Barbero, Finite element analysis of composite materials, CRC Press, Boca Raton, 2008.

[3] EJ Barbero, Introduction to Composite Materials Design, Second Edition ed., CRC Press, Boca Raton, 2010.

[4] RJ Nuismer, SC Tan. Constitutive Relations of a Cracked Composite Lamina, Journal of Composite Materials. 22 (1988) 306-321.

[5] SC Tan, RJ Nuismer. A Theory for Progressive Matrix Cracking in Composite Laminates, Journal of Composite Materials. 23 (1989) 1029-1047.

[6] GJ Dvorak, N Laws, M Hejazi. Analysis of Progressive Matrix Cracking in Composite Laminates I. Thermoelastic Properties of a Ply with Cracks, Journal of Composite Materials. 19 (1985) 216-234.

[7] BN Nguyen. A Three-Dimensional Modeling of Transverse Matrix Cracking in Laminated Composites, Key Eng Mat. 127-131 (1997) 1117.

[8] N Laws, GJ Dvorak, M Hejazi. Stiffness changes in unidirectional composites caused by crack systems, Mech.Mater. 2 (1983) 123-137.

[9] J Varna, R Joffe, NV Akshantala, R Talreja. Damage in composite laminates with off-axis plies, Composites Sci.Technol. 59 (1999) 2139-2147.

[10] S Liu, JA Nairn. Formation and propagation of matrix microcracks in cross-ply laminates during static loading, J Reinf Plast Compos. 11 (1992) 158-178.

[11] J Varna, R Joffe, R Talreja. A synergistic damage-mechanics analysis of transverse cracking [ $\pm \Theta / 904] \mathrm{s}$ laminates, Composites Sci.Technol. 61 (2001) 657-665. 

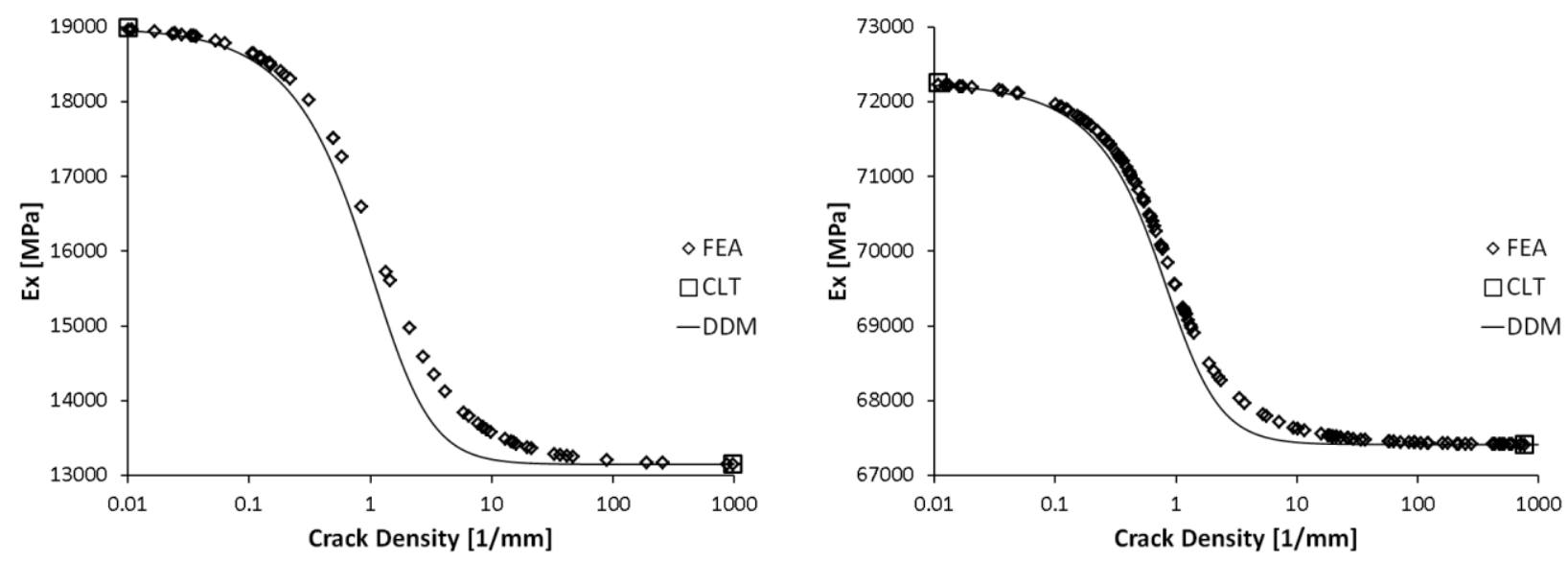

Figure 3.5 Ex vs. Crack density for Laminate 1.

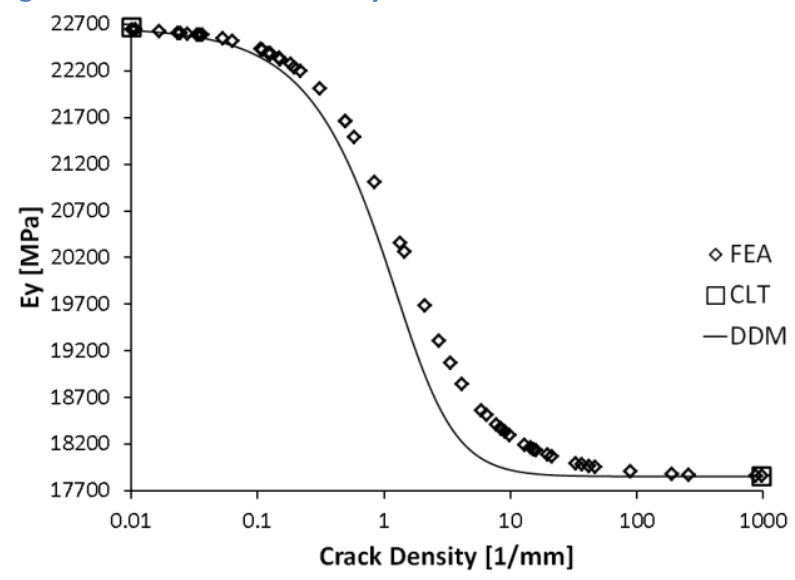

Figure 3.6 Ex vs. Crack density for Laminate 6.

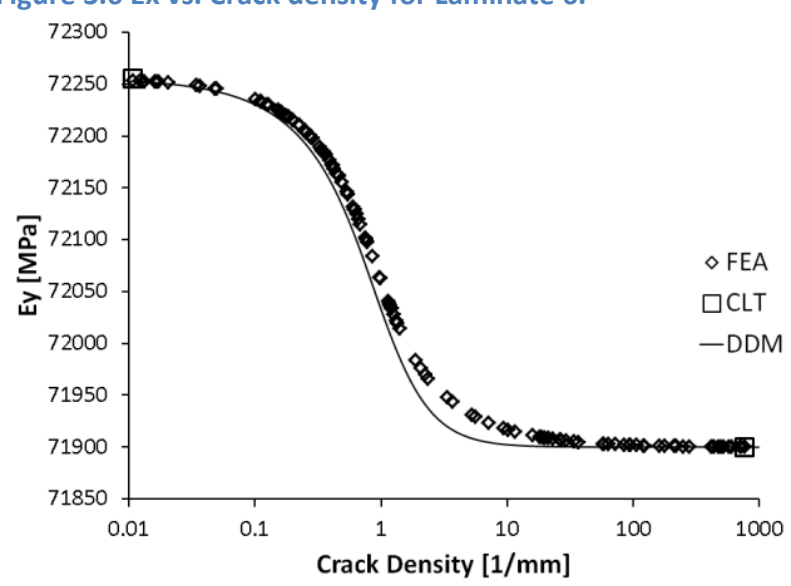

Figure 3.7 Ey vs. Crack density for Laminate 1.

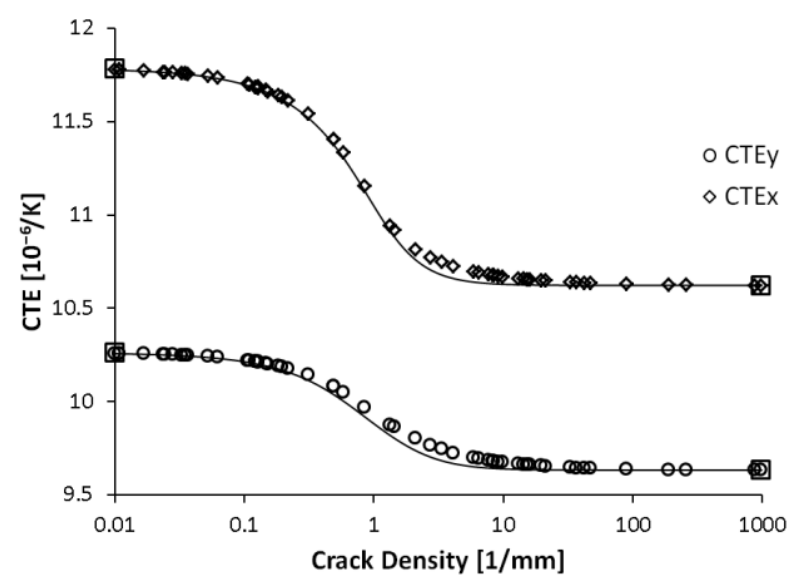

Figure $3.9 \alpha_{x}$ and $\alpha_{y}$ vs. Crack density for Laminate 1.

Figure 3.8 Ey vs. Crack density for Laminate 6.

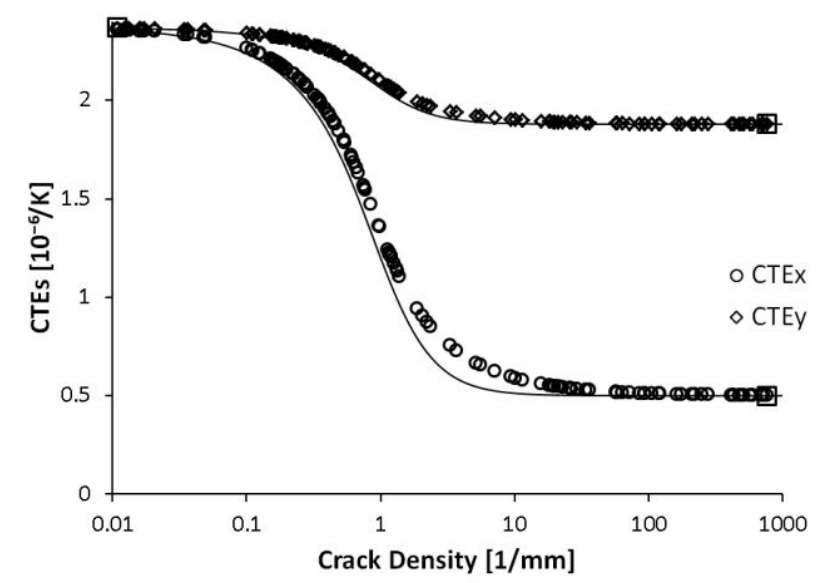

Figure $3.10 \alpha_{x}$ and $\alpha_{y}$ vs. Crack density for Laminate 6. 


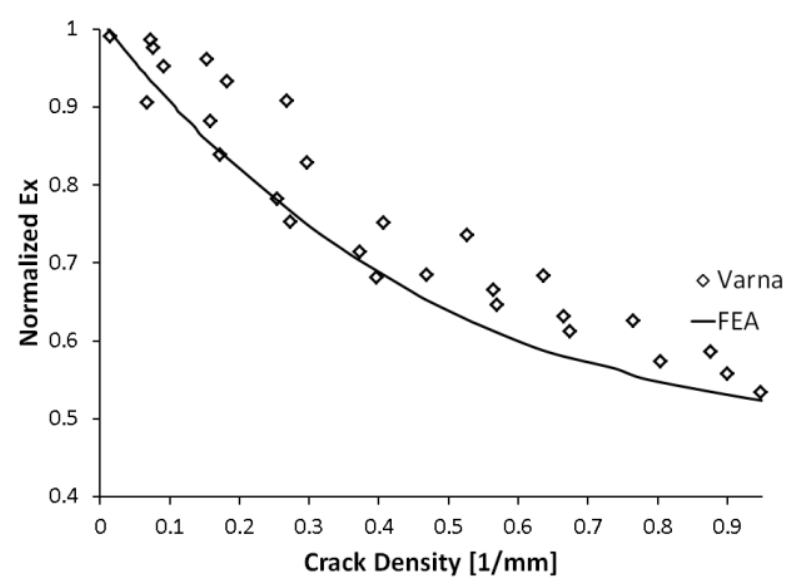

Figure 3.11 Normalized Ex vs. Crack density for Laminate 2.

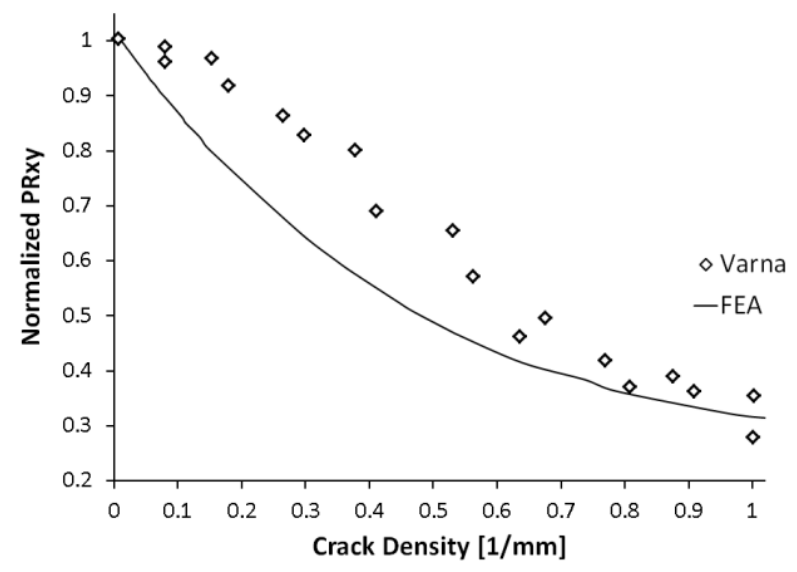

Figure 3.12 Normalized Poisson's Ratio vs. Crack density for Laminate 2.

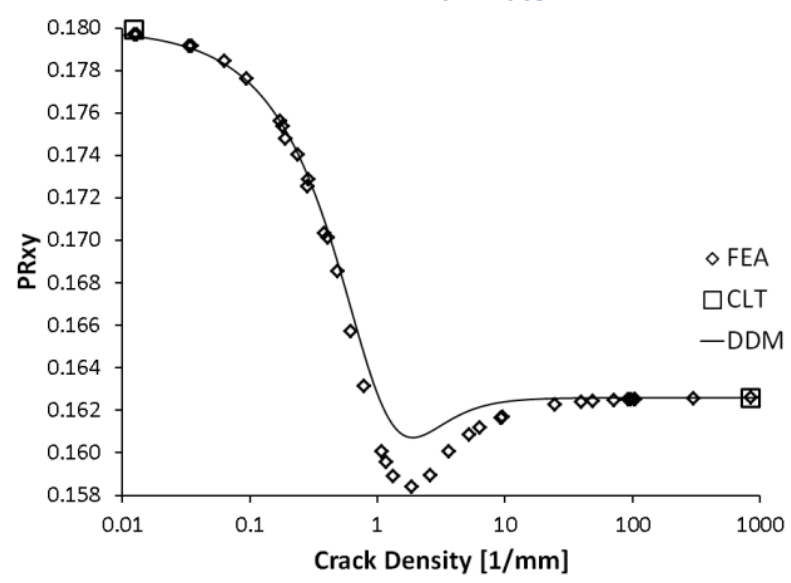

Figure 3.13 Poisson's Ratio vs. Crack density for Laminate 3.

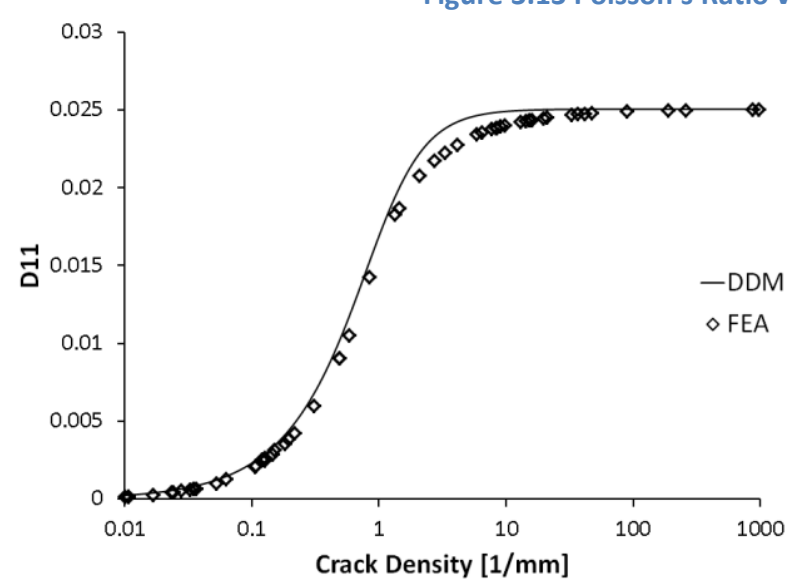

Figure 3.14 $D_{11}$ vs. Crack Density for Laminate 1.

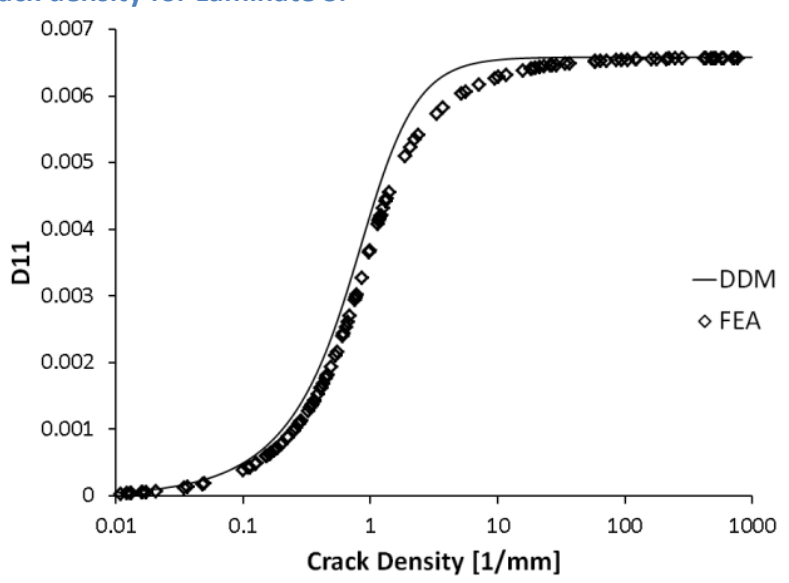

Figure $3.15 D_{11}$ vs. Crack Density for Laminate 6. 

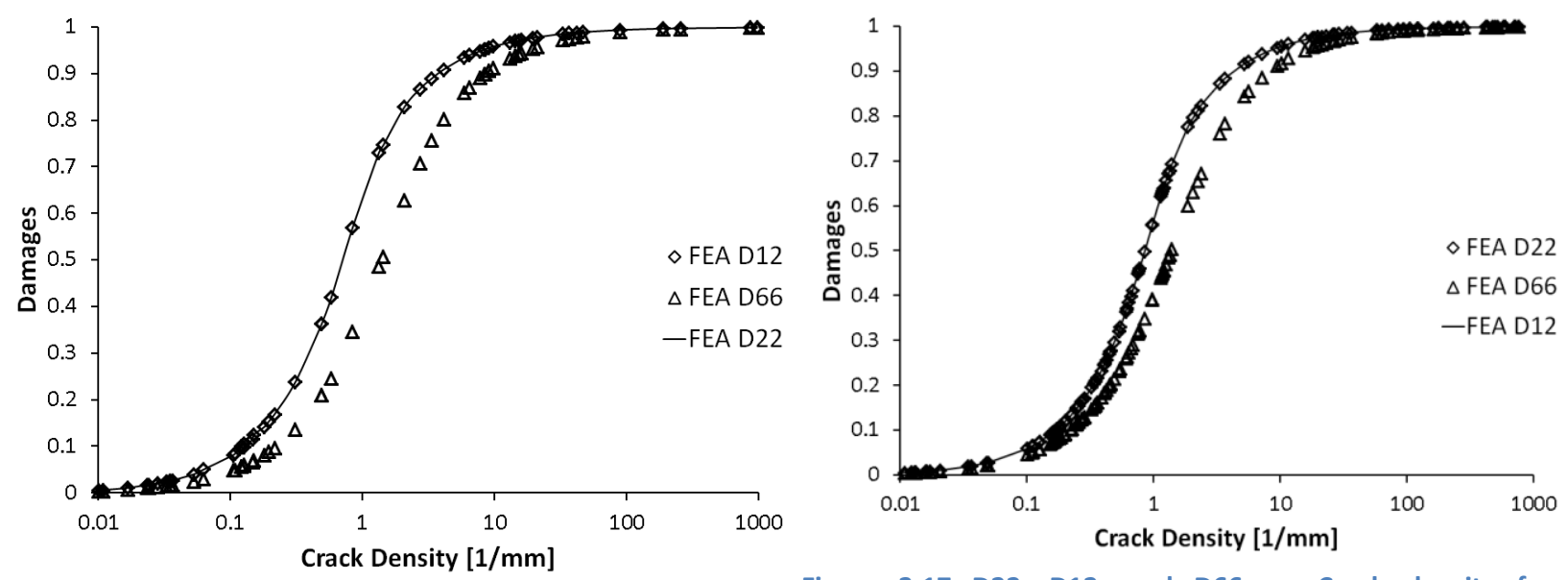

Figure 3.16 D22, D12, and D66 vs. Crack density for Laminate 1.

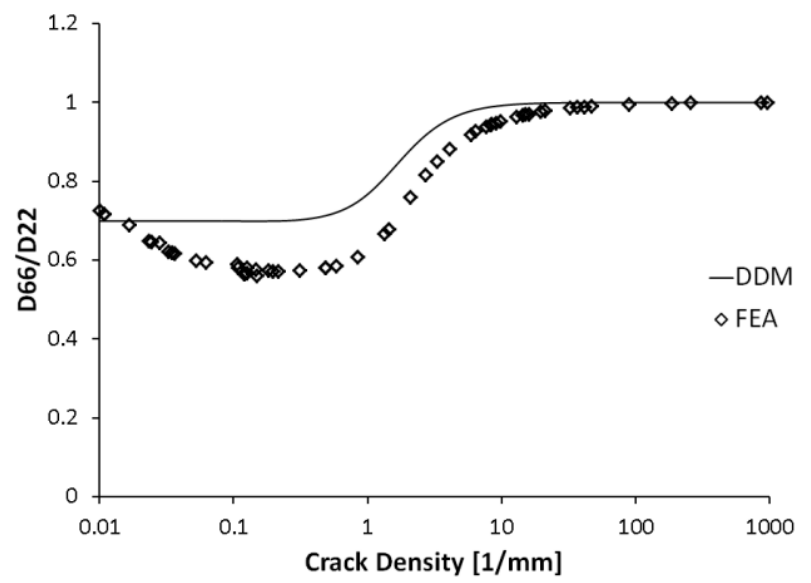

Figure 3.17 D22, D12, and D66 vs. Crack density for Laminate 6.

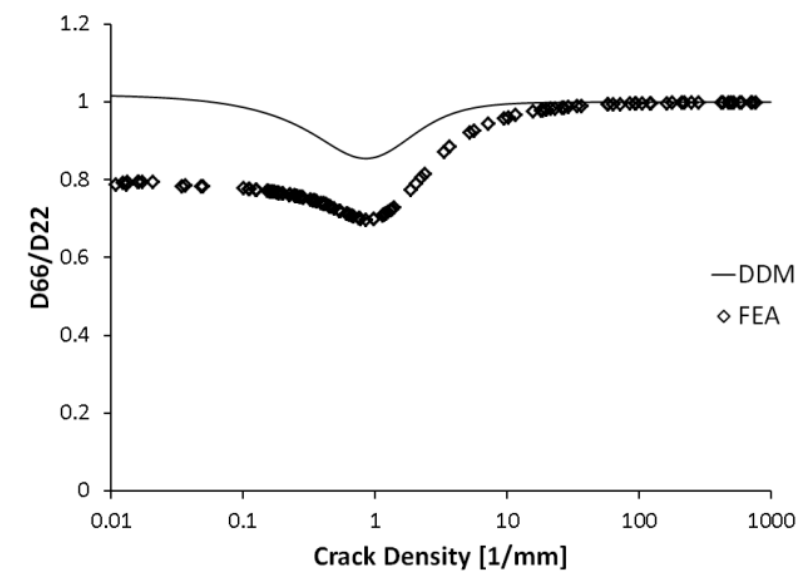

Figure 3.18 D66/D22 vs. Crack Density for Laminate 1.

Figure 3.19 D66/D22 vs. Crack Density for Laminate 6. 


\section{CHAPTER 4. DETERMINATION OF GIC}

The discrete damage mechanics model (DDM) explained in Chapter 2 and validated in Chapter 3 needs at most two parameters to completely predict damage initiation and evolution. They are the fracture toughness in mode one and two. The question arises then how to obtain these parameters, since there is no experiment that can readily measure the values of these material properties. The following approach is here introduced. Available diverse experimental results (Young modulus, Crack density, Poisson's ratio, etc.) are quantitatively compared against the prediction of the model for different values of fracture toughness by a minimization algorithm that looks for the minimum error in this fitting. This procedure is done for each material that needs to be characterized and once the fracture toughness is thus established, it is used to predict and compare different laminates made of the same material to assess the quality of the model predictions.

The constitutive relationship established by the model is intrinsically mesh-independent, since it relies in analytical solutions of the equilibrium equation. Furthermore, the experimental data chosen to adjust the fracture toughness are gathered from uniaxial tensile tests, so the load and strains are uniform. From these two conditions, it is evident that no convergence study is necessary and the model needs only to contain one element to accurately reproduce the test conditions.

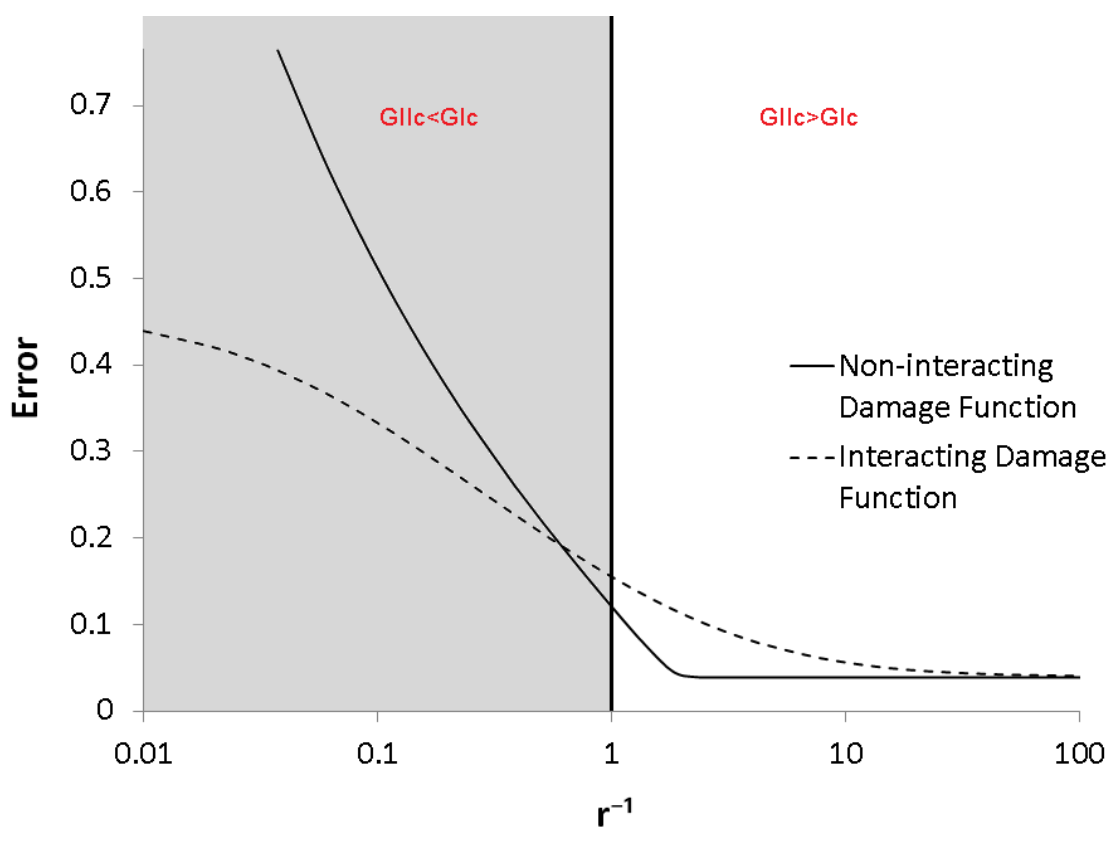

Figure 4.1 Error vs. $G_{I I c}$ normalized by $G_{I c}$ for the interacting and non-interacting damage functions for Laminate 1 (cracks propagate in mode I). The non-interacting damage function converges faster to the minimum error. Grayed zone correspond to hypothetical values, experimentally $G_{I I c}>G_{I c}$. 


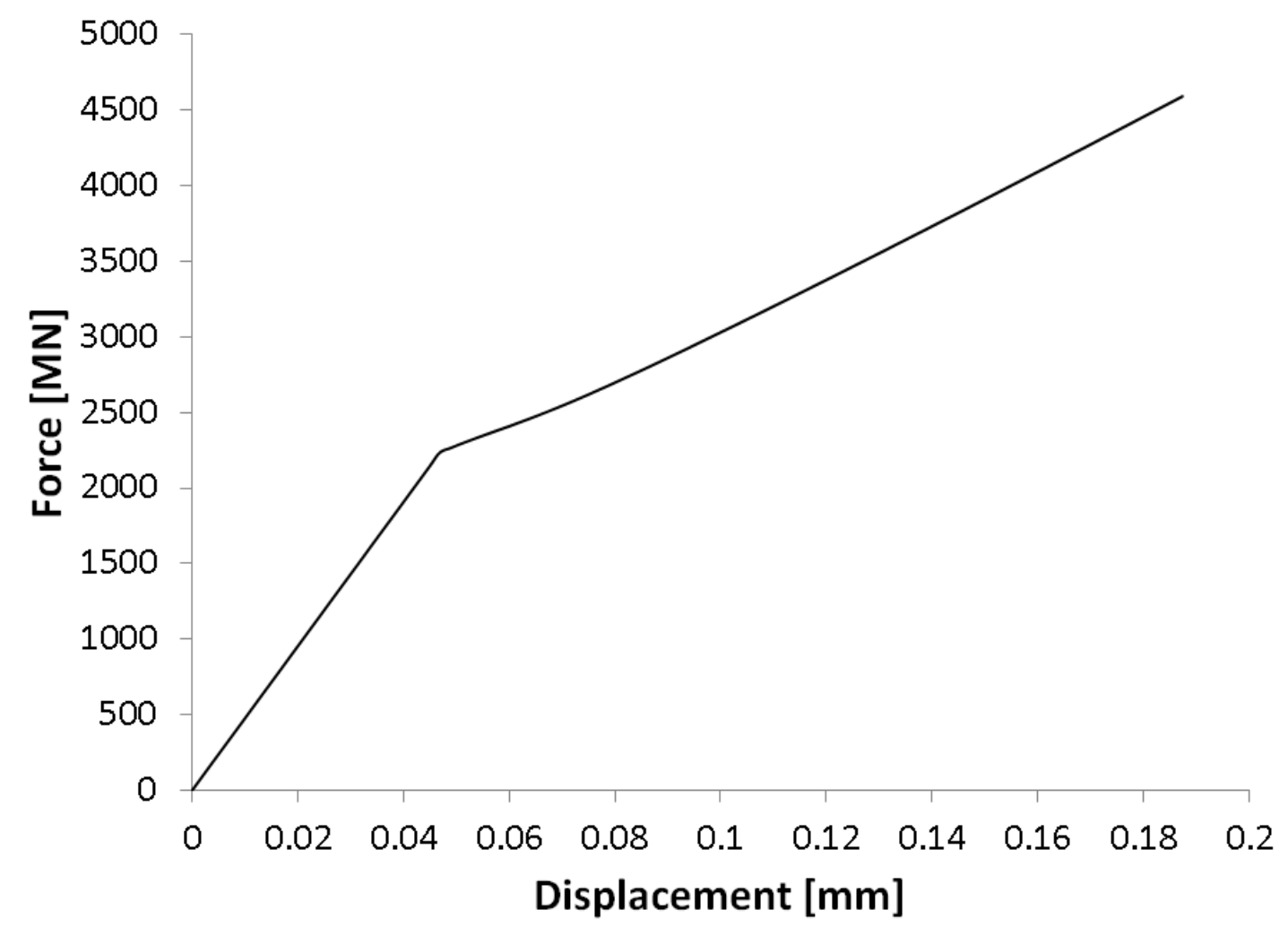

Figure 4.2 Force vs. Displacement for Laminate 2. The solid line is independent (up to single precision) of element type and number.

Nevertheless, and to further test the subroutine, laminate 2 (Table 3.1) was simulated with the combinations shown in Table 4.2

and the results for force and displacement were compared (see Figure 4.2). The difference between simulations is the single precision error (1e-8).

Table $4.1 G_{I c}$ obtained by the minimization algorithm for the materials analyzed.

\begin{tabular}{ll}
\hline Material & $G_{I c}\left[\frac{k J}{m^{2}}\right]$ \\
\hline Fiberite/HyE 9082Af [9] & 0.2535 \\
Avimid ${ }^{\circledR}$ K Polymer/IM6 [10] & 0.2580 \\
Fiberite 934/T300 [10] & 0.2080 \\
Hercules 3501 -6/AS4 [9] & 0.0600 \\
\hline
\end{tabular}


Table 4.2 Element type and number tested.

\begin{tabular}{|lr|}
\hline Element type & Number of elements \\
\hline S4R5 & 1 and 10000 \\
\hline S8R5 & 1 \\
\hline S8R & 1 \\
\hline S4R & 1 \\
\hline S3 & 2 \\
\hline S3R & 2 \\
\hline STRI65 & 2 \\
\hline
\end{tabular}

The model is implemented in an Abaqus user subroutine (UGENS) that models the shell section properties. An axial displacement is applied to the edge of one S4R element to apply the desired strain or stress (dependent on the experimental results available).

The measure of the distance between experimental data and the model predictions is given by

$$
\operatorname{Error}\left(G_{I c}, G_{I I C}\right)=\frac{1}{N} \sqrt{\sum_{i=1}^{N}\left[P^{D D M}\left(x_{i}\right)-P^{E x p}\left(x_{i}\right)\right]^{2}}
$$

Where $P^{E x p}$ is any property reported in the experiment (Normalized Young Modulus, etc), $\mathrm{x}$ is the test progress indicator (Strain, Stress, Crack Density, etc), $\mathrm{N}$ is the number of experimental points available and $P^{D D M}$ is the prediction by DDM of the property of interest.

Note that the error is used only to quantitatively asses the goodness of the prediction for a particular laminate. This allows us to use a mathematical algorithm to seek for the best fit and thus automate the search for the energy release rates. But the value on its own is not useful to determine if a fit was successful or not, in the sense that the model is able to predict or not the behavior of the laminate past the damage initiation. This latter assessment is somewhat qualitatively and would depend on the accuracy desired by the designer, but always taking into account the stochastic nature of the cracking events and leaving room for uncertainties.

The error is in general a function of $G_{I c}$ and $G_{I I C}$, but if the conditions of the test where such as to propagate cracks only by a mode, say mode I, then $G_{I C}$ dominates the error and $G_{I I c}$ has negligible effect. The damage activation function used in the model determines how quickly the minimization algorithm converges to a pair of values for $G_{I C}$ and $G_{I I c}$. When the interacting damage function is used the error varies smoothly with respect to $r$ (see Figure 4.1). In contrast, for the non-interacting damage function the error is minimum for any value of $G_{I I C}$ that exceeds a threshold. Which represents better what actually happens, namely the cracks propagate in mode I and $G_{I I C}$ should at least be the threshold value. In order to determine the actual value of $G_{I I C}$, experiments where cracks propagated in mode II should be available. 
It is concluded, that when only mode $\mathrm{I}$ is acting on a laminate one needs only to find the value of $G_{I C}$ and set $G_{I I C}$ sufficiently large so there is no contribution to the error by this mode. This was found to be the case for all the laminates analyzed in this report.

\subsection{Implementation}

There are several implementation details that are explained in this section.

\subsubsection{Minimization Algorithm}

The minimization algorithm employed in this work is available in Matlab ${ }^{1}$ as a built-in function called fminsearch and it is based in the work of Lagarias et al. [1]

\subsubsection{Measurement of the distance between points}

In order to follow the damage in detail, the strain step employed in the model is very small (controlled by Abaqus, but at most one hundredth of the final strain), while the separation between experimental data points is not controllable, varies between each pair of points and is usually larger than the numerical step (typically there are at most 15 experimental points, so the step is the inverse of that). The problem arises then in how to evaluate (4.1), since the experimental points $x_{i}$ might not necessary coincide with the values of strain calculated by Abaqus. This situation is depicted in Figure 4.3 , the solution to this problem is to interpolate the values of the model $p$ with a spline (cubic interpolation) and evaluate the spline at the experimental data points. Then the error is simply the norm of the Nth dimensional vector of the component wise subtraction between the vector containing the experimental values of $p$ at $x_{i}$ and the vector containing the interpolated values of the model prediction for $p$ at $x_{i}$. This procedure can be easily implemented in Matlab.

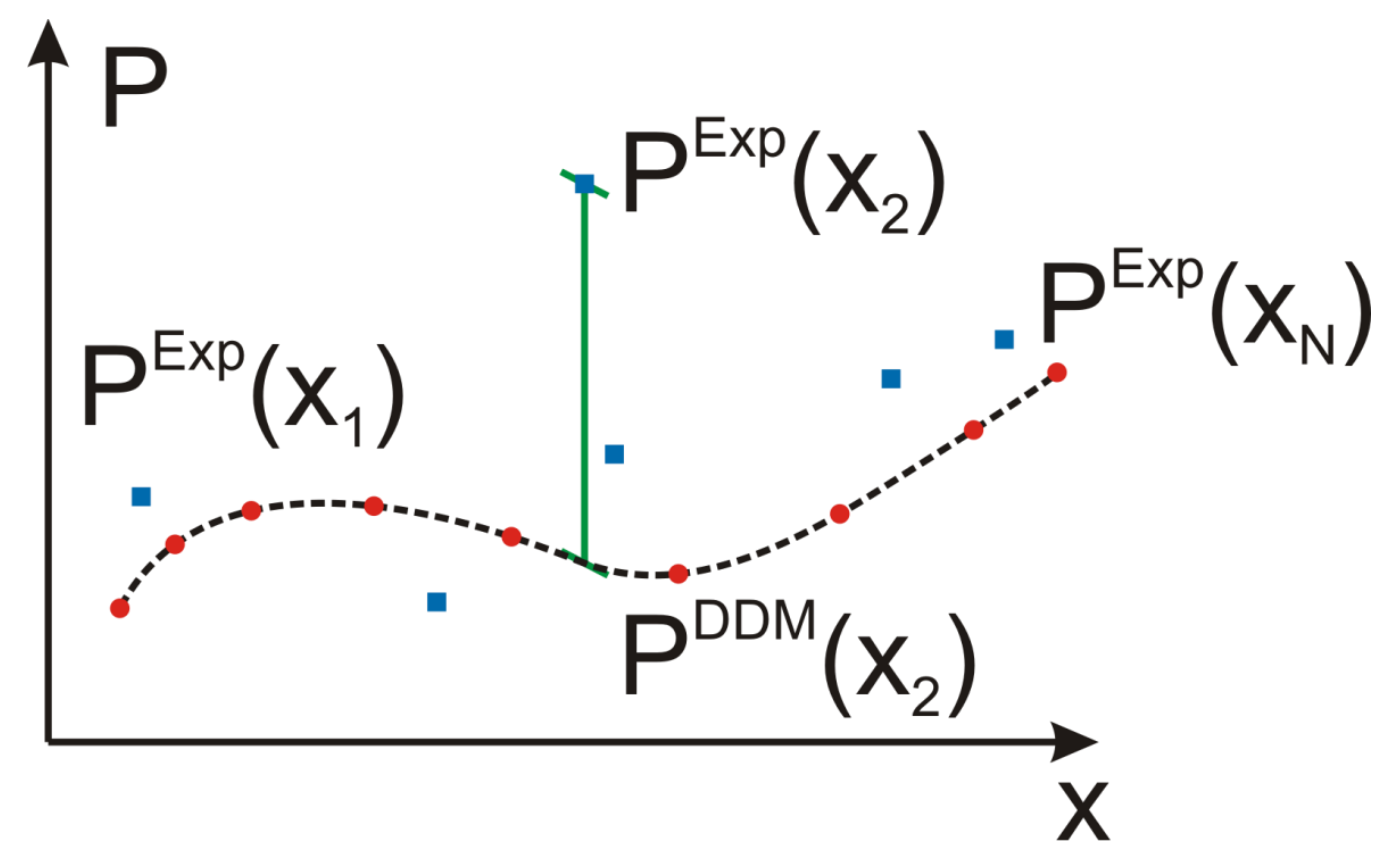

Figure 4.3 Generic P vs. X plot. Squares are experimental points and circles are Abaqus points. Dashed line is the spline that allows for the evaluation of $\mathrm{P}^{\mathrm{DDM}}$ at the same value of experimental $\mathrm{x}$.

\footnotetext{
${ }^{1}$ MATLAB is a registered trademark of The MathWorks, Inc.
} 


\subsubsection{Parameterization of the model}

To effectively run the different cases, the model was parameterized in material properties, stacking sequence (orientation and thickness), $G_{I C}, G_{I I c}, \Delta T$, and maximum strain. Abaqus/CAE was used interactively while recording the Python commands in the journal file. Next, the journal file was transformed into a function that receives the parameters and writes to disk a file containing the strain, stress, Poisson's ratio, Young modulus and the crack density of each lamina for every value of strain.

As of Abaqus 6.10-2, user general sections are not compatible with CAE, which means that the input file cannot be completely generated by the Python API. A workaround for this issue was implemented where the keywords for the user general section were generated by a specially coded function that writes the properties that need to be passed to the UGENS eight floats per line and injected in the CAE input file by means of keywordBlock functionality.

\subsubsection{Numerical subroutines}

The DDM model requires the calculation of eigenvalues, matrix inversion and other linear algebra operations that have to be computed as efficiently as possible since these operations are done in each Gauss Point. With this in mind, the DDM model was codified to use the Intel implementation of LAPACK and BLAS, namely Intel Math Kernel Library.

\subsubsection{Need for a client-server architecture}

From the previous sections it is clear that Matlab and simultaneously the embedded interpreter of Python in Abaqus have to be used to come up with the optimum value of $G_{I c}$ and later on to make the comparison with the rest of the laminates. There must be a way of passing information back and forth these two programs. The best way to achieve this is by employing internet sockets, a reduced protocol had to be devised to regulate this communication that is depicted in Figure 4.4.

\subsection{Results}

The Abaqus subroutine was employed for the four materials listed in Table 3.2. The property $p$ and the progress indicator $x$ varied for each case and are mentioned below. The error between the model and the experimental data can be found in each figure. The value of $G_{I c}$ for the different materials in summarized in Table 4.1.

\subsubsection{Fiberite/HyE 9082Af}

The available experimental data for this set of laminates is crack density vs. longitudinal strain for Laminates 1-3. In (4.1), the property is crack density and the test progress indicator is the strain. For laminates 15-18 the independent variable is again strain but the dependent variable is the Normalized modulus and the Normalized Poisson's ratio.

Laminate 2 was used to determine the value of $G_{I C}$ for this material. The best fit can be seen in Figure 4.6. The error is extremely small and is due to the dispersion of the experimental results exclusively. With the value of $G_{I C}$ reported in Table 4.1 the DDM model was used to simulate Laminate 1 (Figure 4.5) and Laminate 3 (Figure 4.7), presenting good agreement between DDM and the experiments. 
Figures 4.19-26 show the results for laminates 15-18. The damage onset coincides for all the cases and the evolution follows a similar trend also.

\subsubsection{Avimid @ K Polymer/IM6}

Nairn reported stress as the independent variable, $\mathrm{x}$ in (4.1), and crack density as the dependent variable, $\mathrm{p}$ in (4.1). Laminate 5 was used to adjust $G_{I C}$ and the result for the final iteration of the minimization algorithm is shown in Figure 4.9, again the prediction is good. Laminate 4, 6, and 7 were simulated with the $G_{I c}$ previously adjusted. Damage in Laminate 4 is observed before the prediction of DDM and the same occurs for Laminate 6 and 7.

\subsubsection{Fiberite 934/T300}

Laminate 12 is used by the minimization algorithm to find the optimum value of $G_{I C}$, the result is shown in Figure 4.16. Laminates 8-11 are then simulated. Damage is predicted by DDM before it is observed for laminates 8 (Figure 4.12) and 9 (Figure 4.13). An extremely good prediction is found for Laminate 10 (Figure 4.14). For Laminate 11 the crack density calculated by DDM is larger than what is reported in the experiments.

\subsubsection{Hercules 3501 -6/AS4}

Laminate 13 is used for the fitting and the result is shown in Figure 4.17. DDM over estimates the crack density for this case and also for Laminate 14 (Figure 4.18).

\subsection{Conclusions}

Overall, DDM follows very closely the experimental results for e-glass and carbon fibers reinforced composites, with the exception of laminates 13 and 14, which can be attributed to the stochastic nature of failure. In any case, more samples of the same laminate should have been tested in order to definitely conclude that DDM is not predicting accurately or what other reason might be behind this disagreement.

Additionally, it has been shown that the non-interacting damage function converges faster than the interacting one to the case when only one mode is operating in the cracked lamina.

\subsection{References}

[1] JC Lagarias, JA Reeds, MH Wright, PE Wright. Convergence properties of the Nelder-Mead simplex method in low dimensions, SIAM Journal on Optimization. 9 (1999) 112-147. 


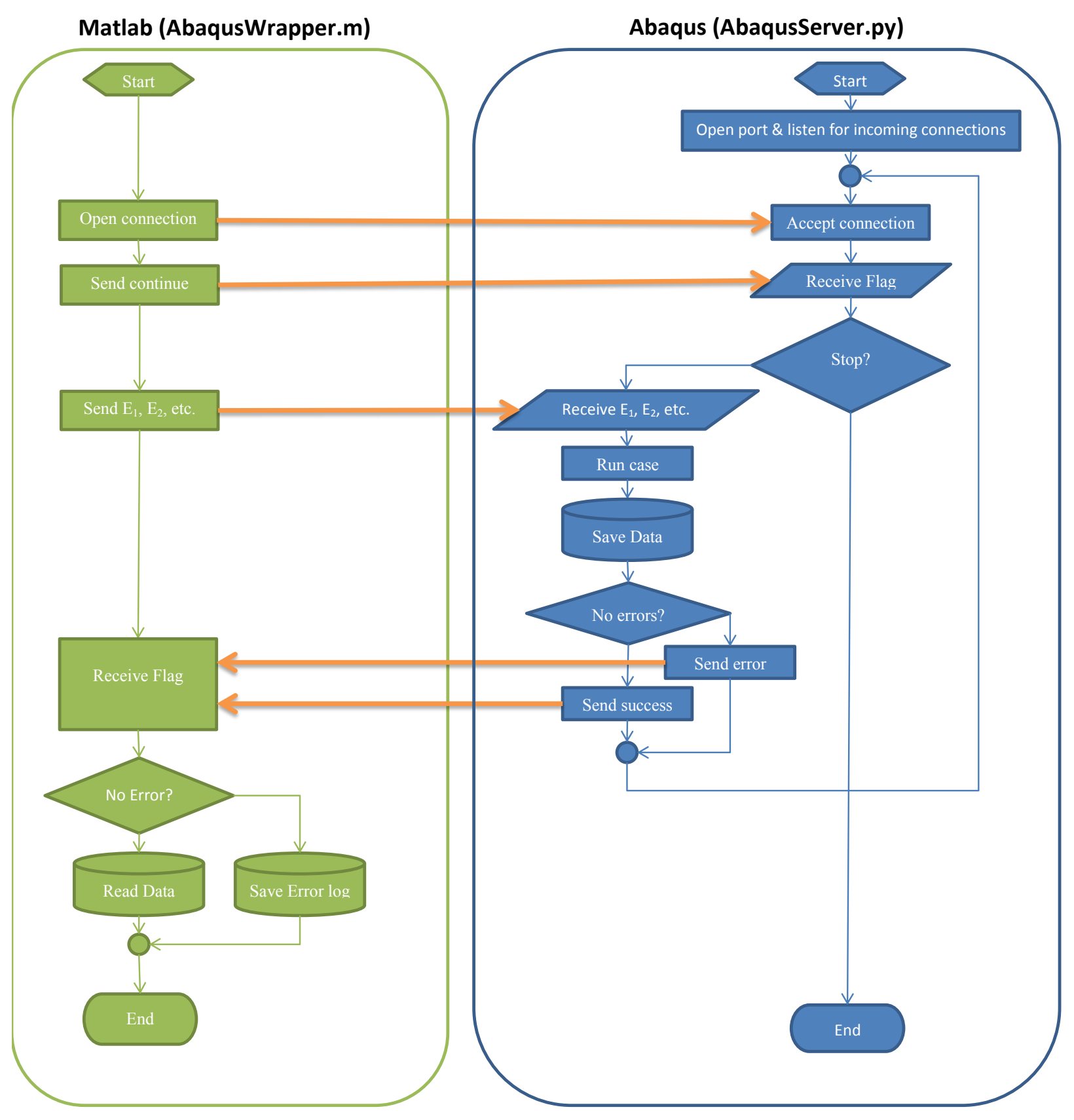

Figure 4.4 Flowchart for the Abaqus, Matlab client server tcp/ip communication. 


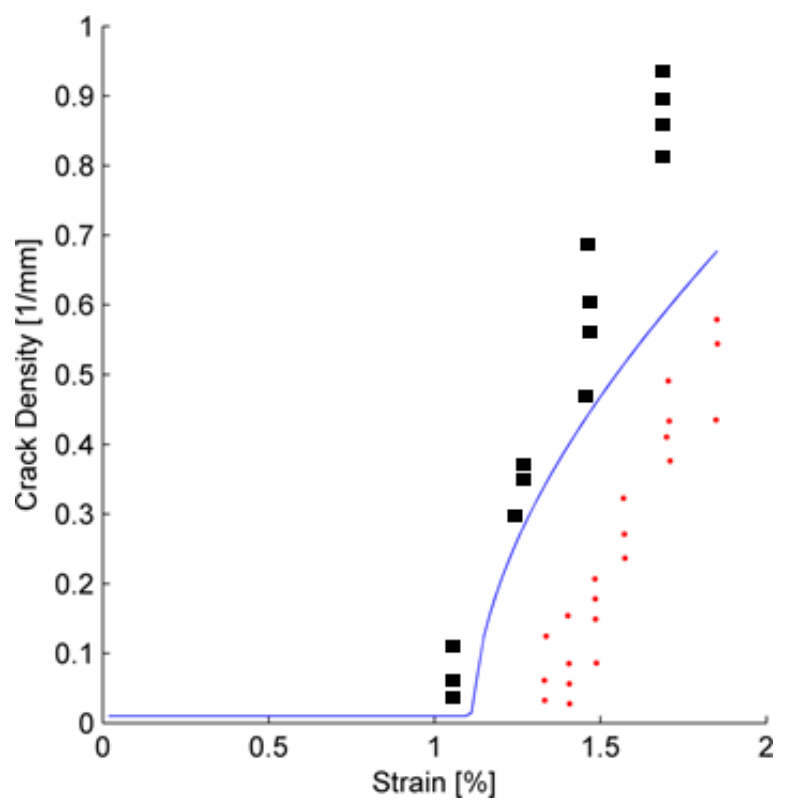

Figure 4.5 Crack Density (P) vs. Strain (x) for Laminate 1. Error calculated as in (4.1) is 0.038991 . Squares are experiment set 1 , circles are experiment set 2 , solid line is the DDM prediction.

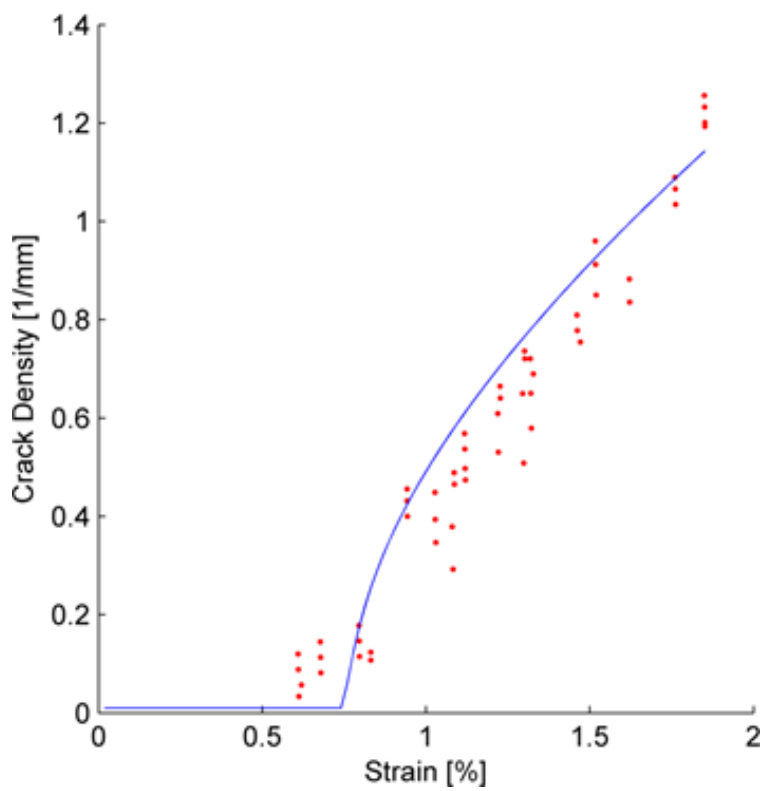

Figure 4.7 Crack Density vs. Strain for Laminate 3. Error is 0.015094 .

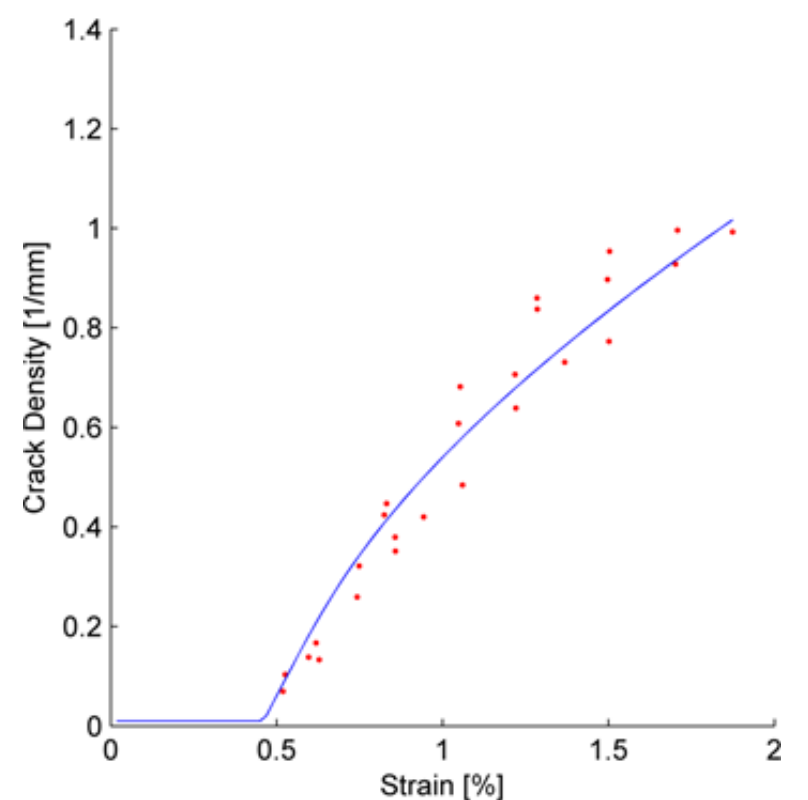

Figure 4.6 Crack Density vs. Strain for Laminate 2. Error is 0.013384 .

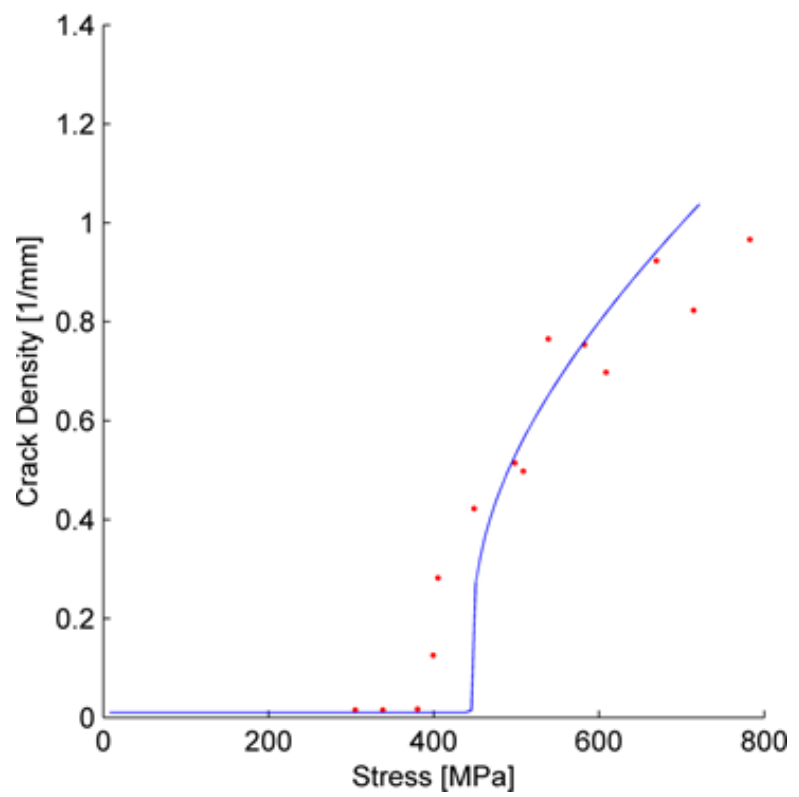

Figure 4.8 Crack Density (P) vs. Stress (x) for Laminate 4. Error is $\mathbf{0 . 0 3 5 8 3 9}$. 


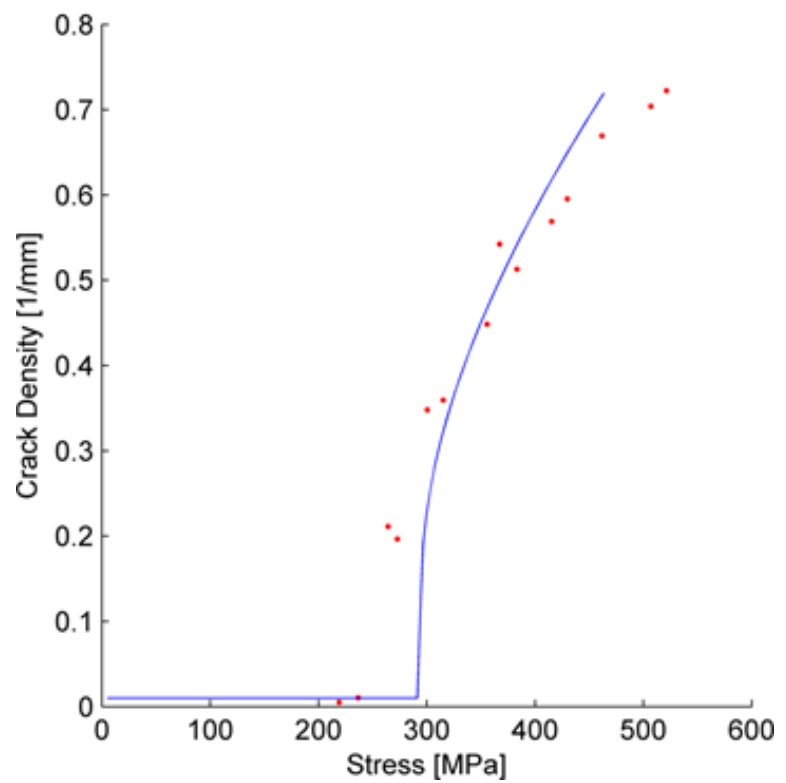

Figure 4.9 Crack Density vs. Stress for Laminate 5. Error is 0.026321 .

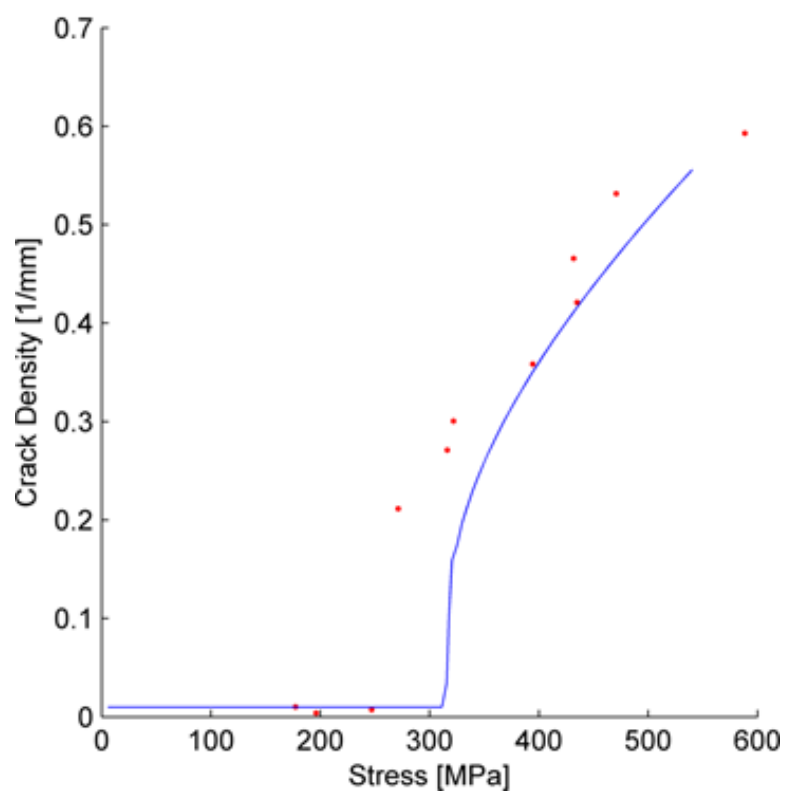

Figure 4.11 Crack Density vs. Stress for Laminate 7. Error is 0.031198 .

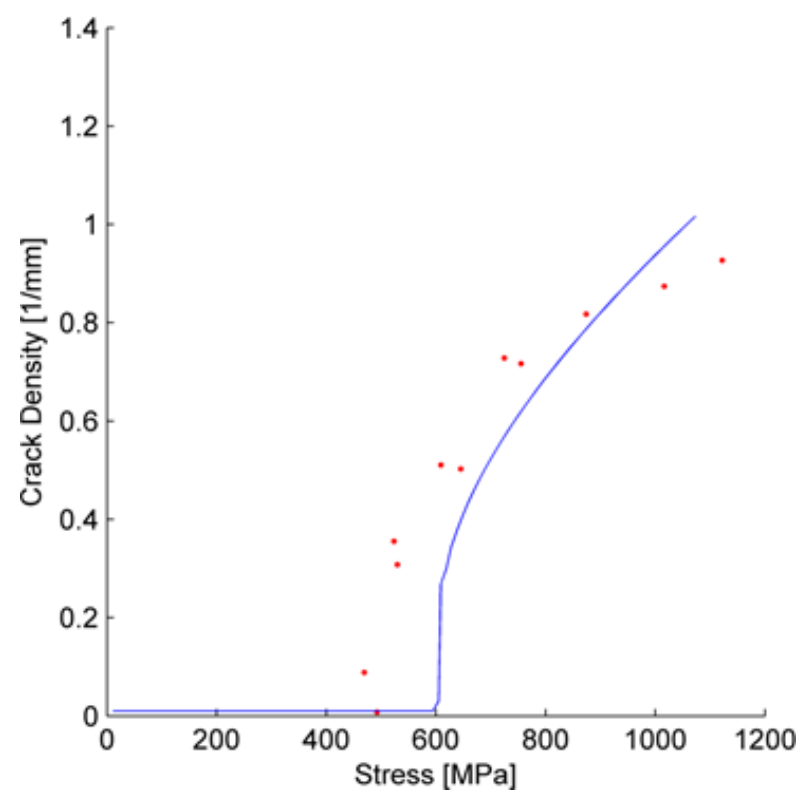

Figure 4.10 Crack Density vs. Stress for Laminate 6. Error is 0.053478 .

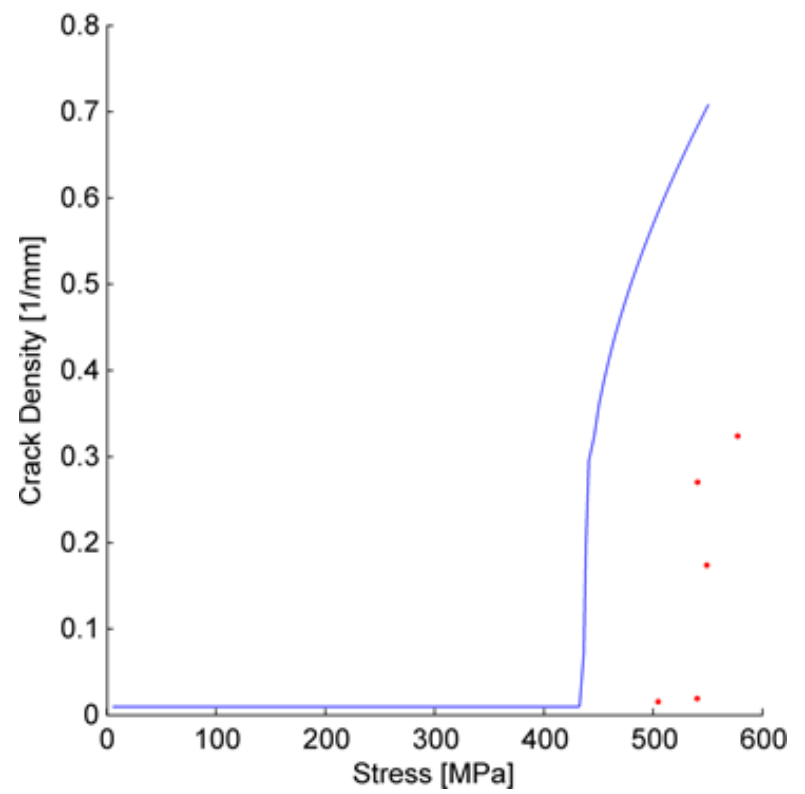

Figure 4.12 Crack Density vs. Stress for Laminate 8. Error is 0.23779 . 


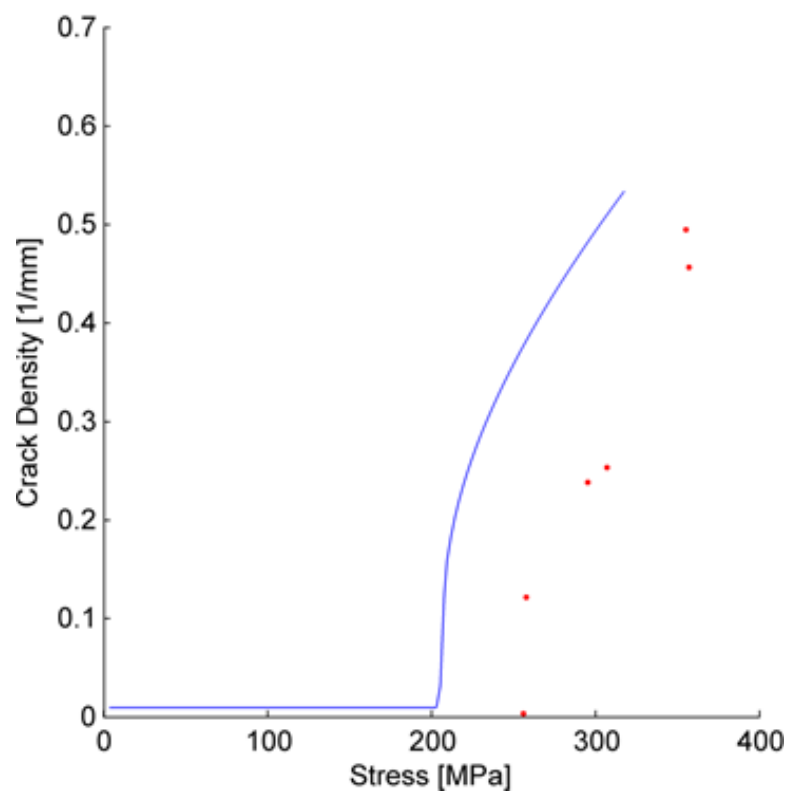

Figure 4.13 Crack Density vs. Stress for Laminate 9. Error is 0.10169 .

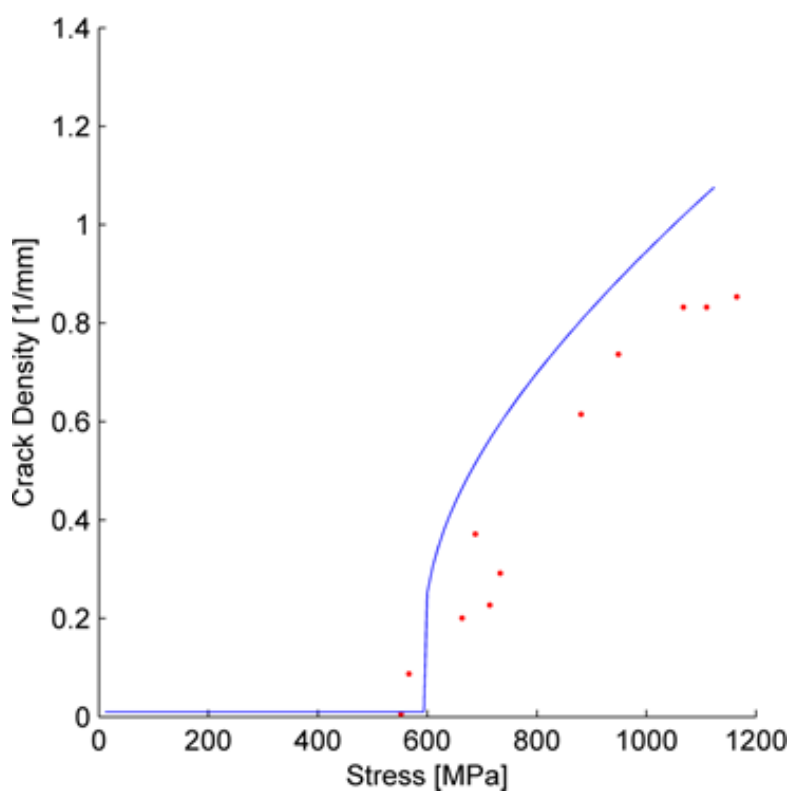

Figure 4.15 Crack Density vs. Stress for Laminate 11. Error is 0.065441 .

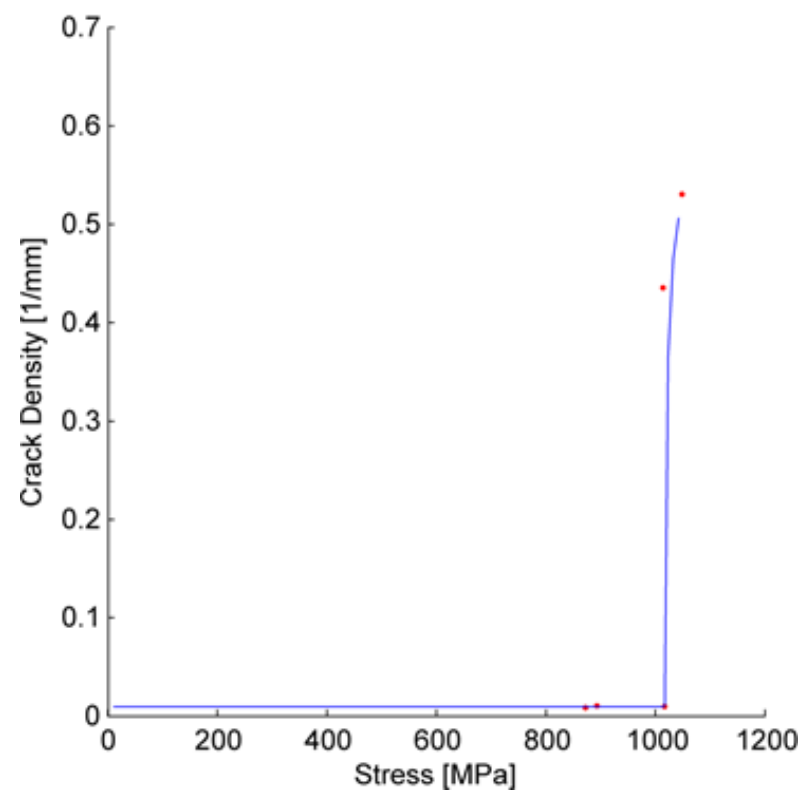

Figure 4.14 Crack Density vs. Stress for Laminate 10. Error is 0.13402 .

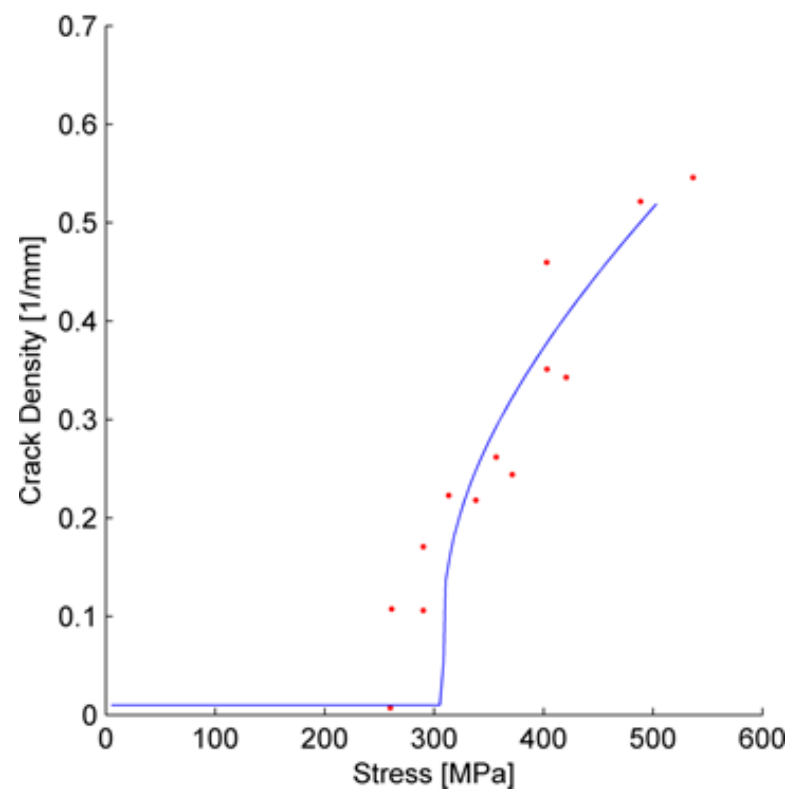

Figure 4.16 Crack Density vs. Stress for Laminate 12. Error is 0.019982 . 


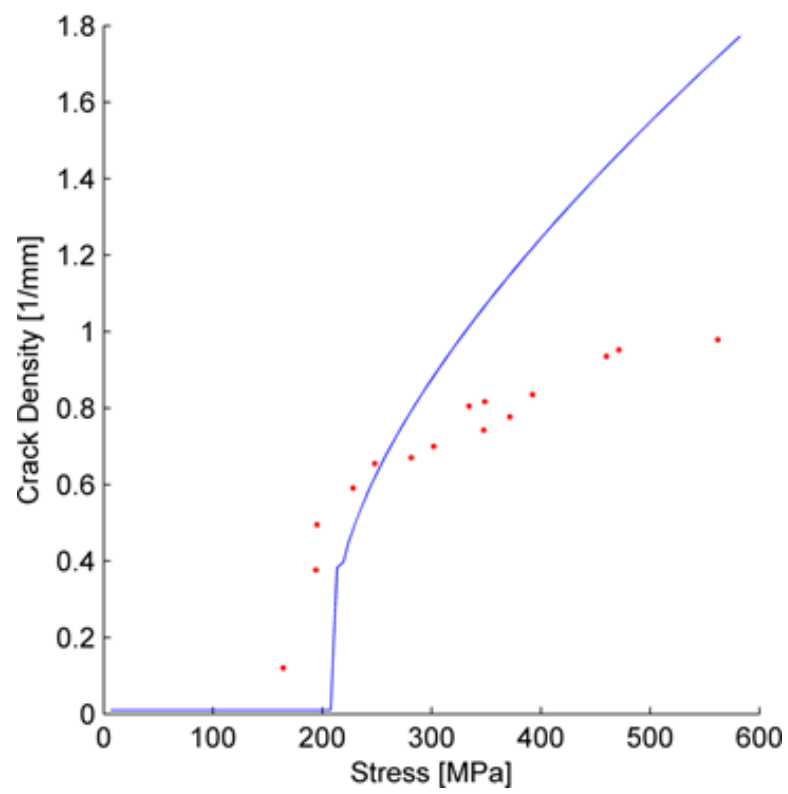

Figure 4.17 Crack Density vs. Stress for Laminate 13. Error is 0.094112

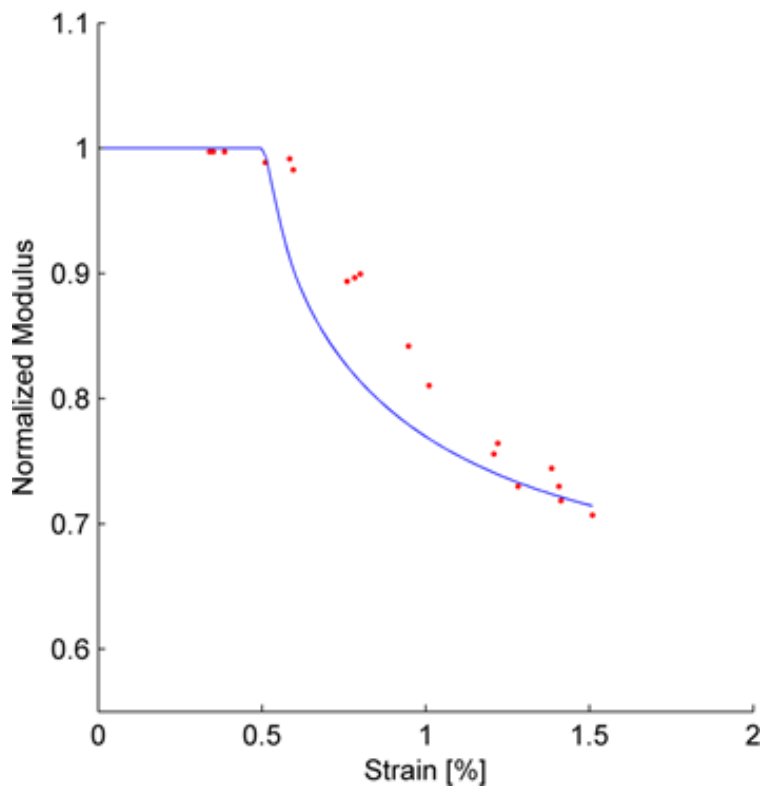

Figure 4.19 Normalized Ex vs. Strain for Laminate 15. Error is 0.010807

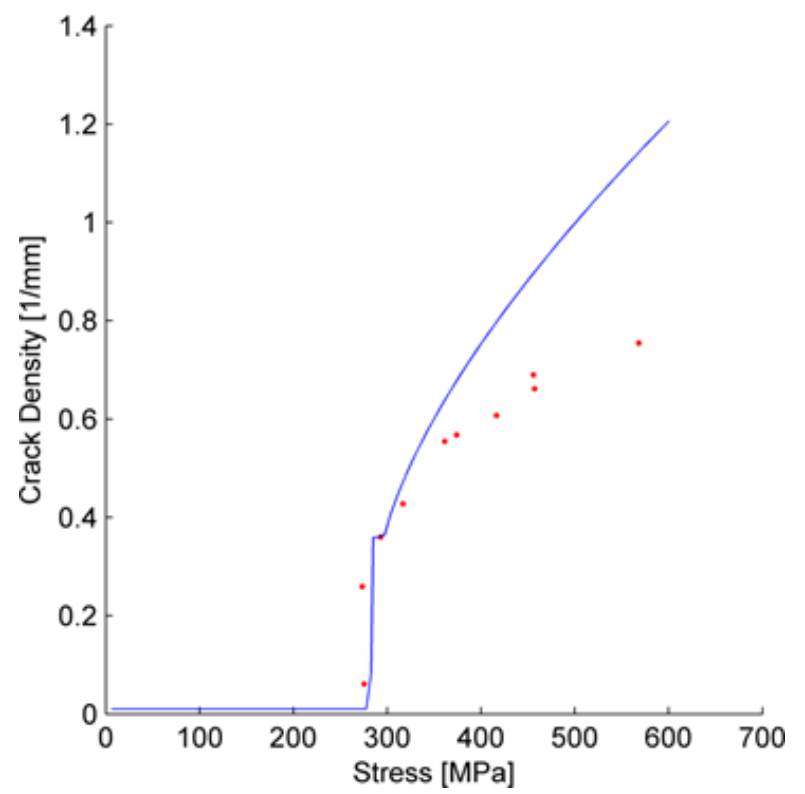

Figure 4.18 Crack Density vs. Stress for Laminate 14. Error is 0.060402 .

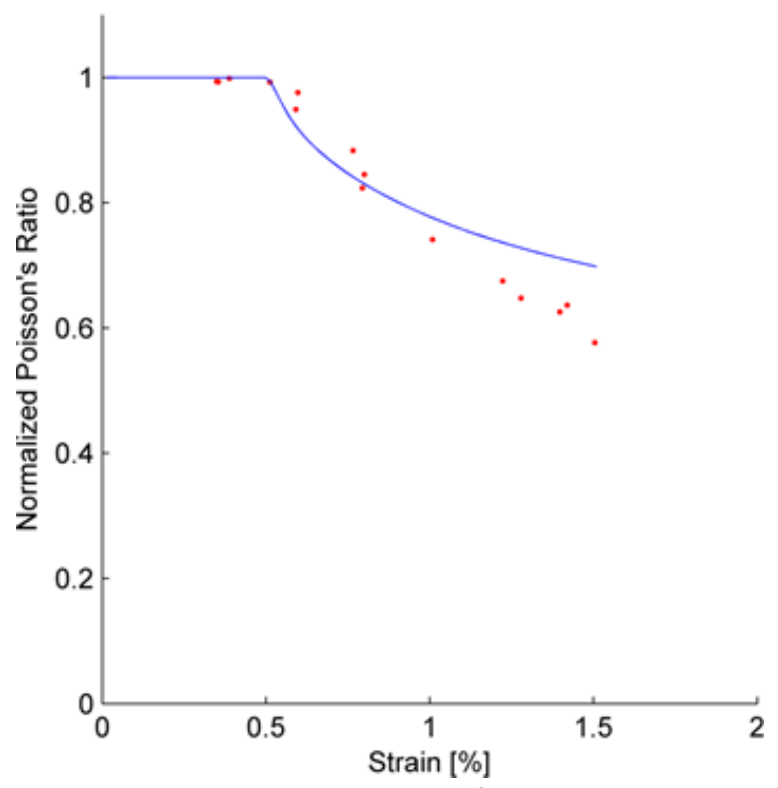

Figure 4.20 Normalized Poisson's ratio vs. Strain for Laminate 15. Error is 0.014131 . 


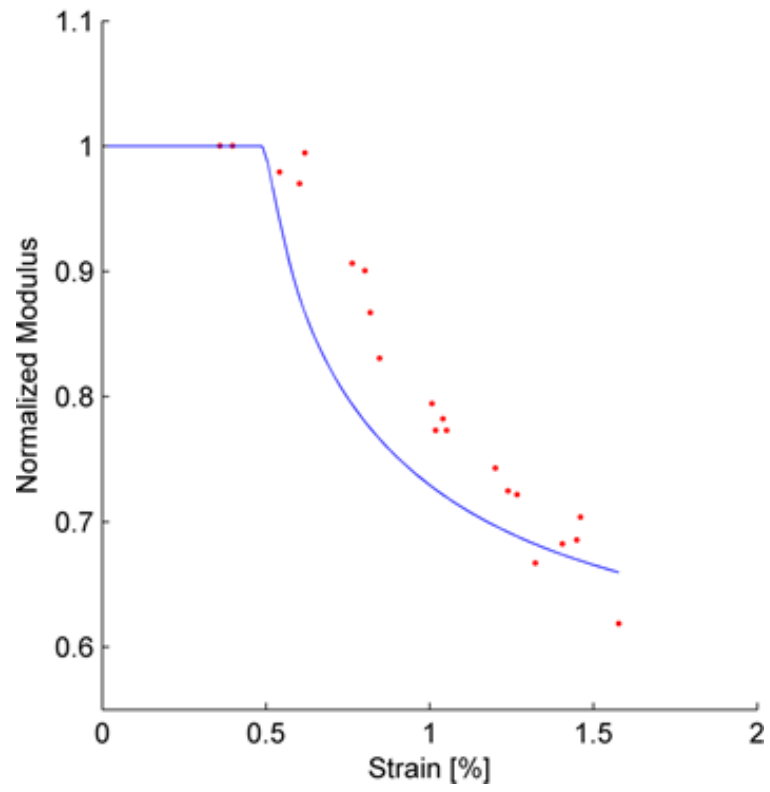

Figure 4.21 Normalized Ex vs. Strain for Laminate 16. Error is 0.01405 .

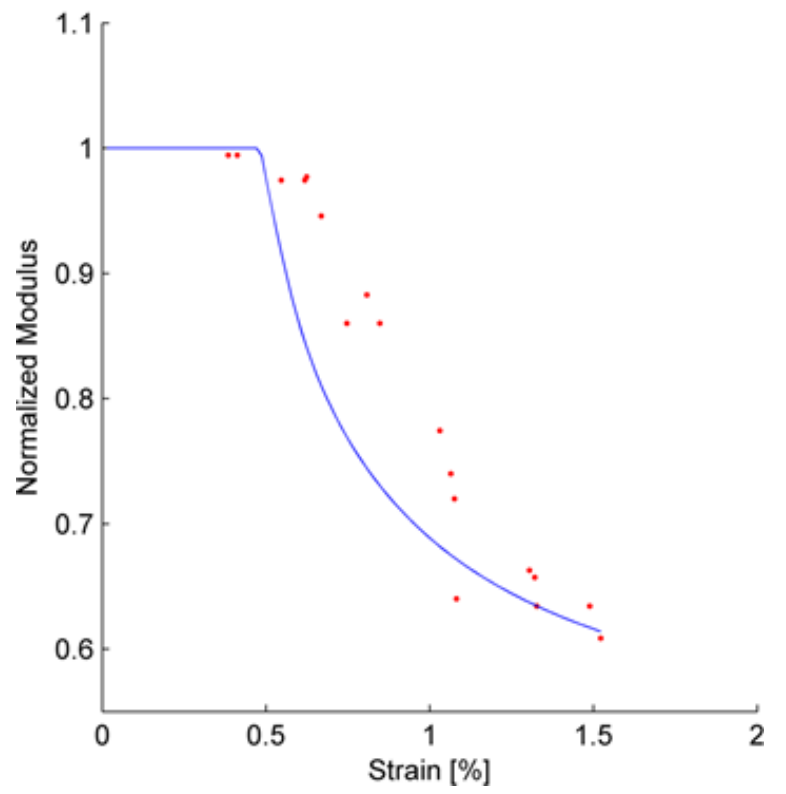

Figure 4.23 Normalized Ex vs. Strain for Laminate 17. Error is 0.019139 .

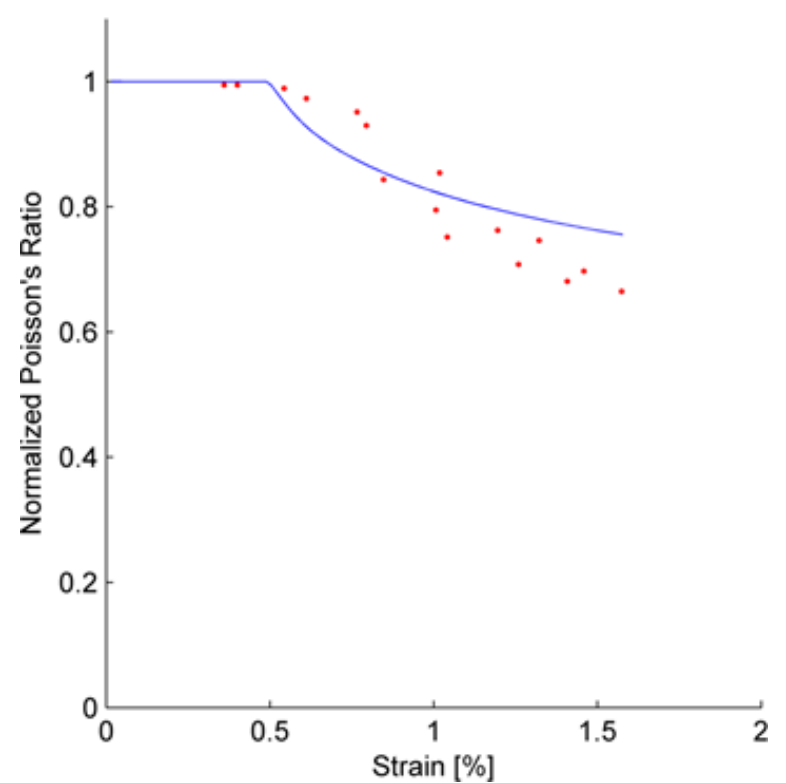

Figure 4.22 Normalized Poisson's ratio vs. Strain for Laminate 16. Error is $\mathbf{0 . 0 1 3 7 5 6 .}$

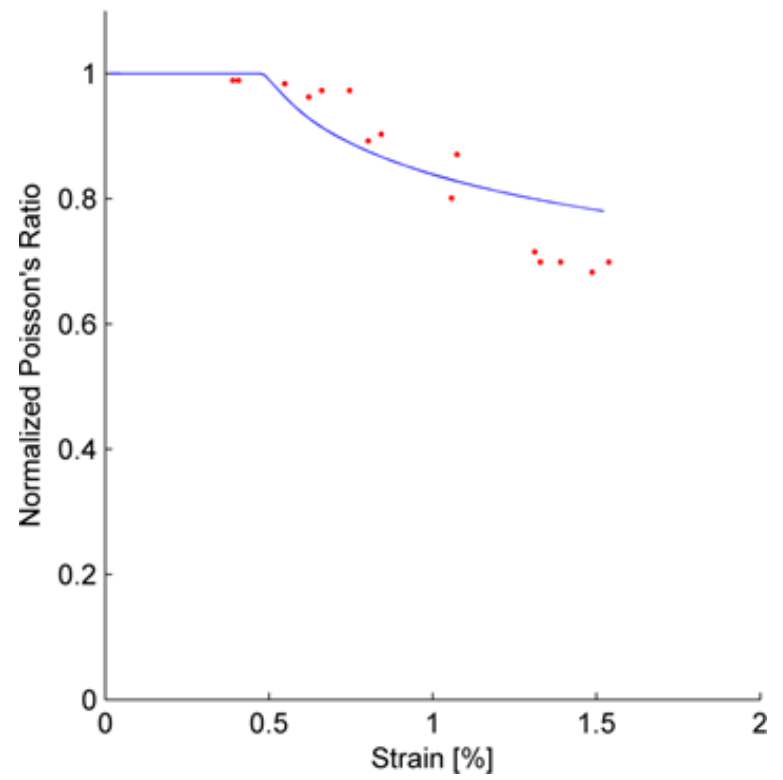

Figure 4.24 Normalized Poisson's ratio vs. Strain for Laminate 17. Error is $\mathbf{0 . 0 1 6 1 3 5}$. 


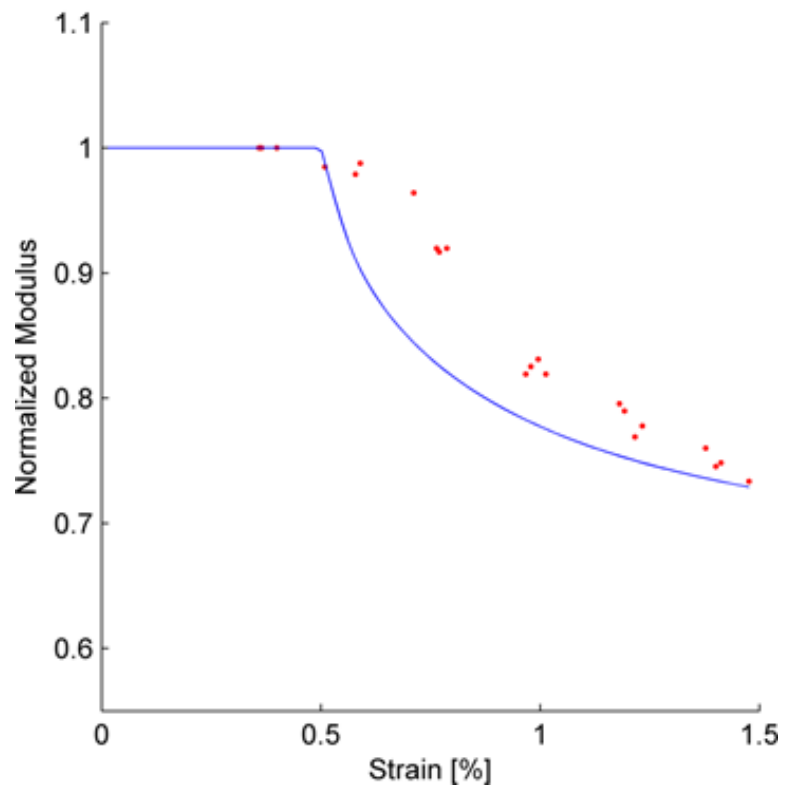

Figure 4.25 Normalized Ex vs. Strain for Laminate 18. Error is 0.011684 .

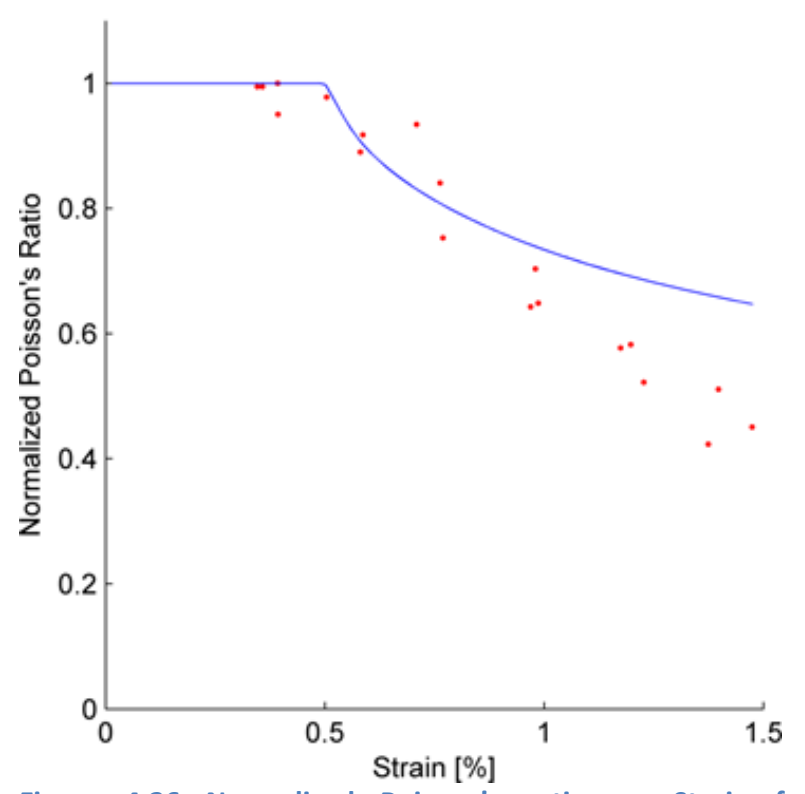

Figure 4.26 Normalized Poisson's ratio vs. Strain for Laminate 18. Error is 0.023928 . 


\section{CHAPTER 5 CONCLUSIONS}

The reduction of laminate in-plane stiffness and coefficient of thermal expansion due to transverse matrix cracking in laminate composite materials is characterized in the present work. For this, a discrete damage mechanics (DDM) model was implemented as a user subroutine in Abaqus. The model is thoroughly described in Chapter 2.

The model was found to agree with finite element analysis (FEA) simulations as shown in Chapter 3. In the limiting cases of intact and completed degraded material properties the model and the FEA results agree with classical laminate theory (CLT). The homogenized lamina properties are also compared between FEA and DDM and it is found that the coefficient of thermal expansion does not change with temperature for the analyzed cases. The components 12 and 22 of the damage tensor are found to be numerically equal for all the simulations.

The prediction of the model for crack density vs. strain and stress is found to be good, as it is shown in Chapter 3 . The energy release rate is satisfactory fitted using a minimization algorithm.

The preferred damage activation function when only one crack opening mode is present is the noninteracting one because it converges to the right energy release rate value in less iterations.

The present work is the starting point to consider delamination in composites because transverse cracks act as stress concentrators where delamination occurs. Once transverse matrix cracking has been characterized, delamination can be treated. 


\section{APPENDIX A}

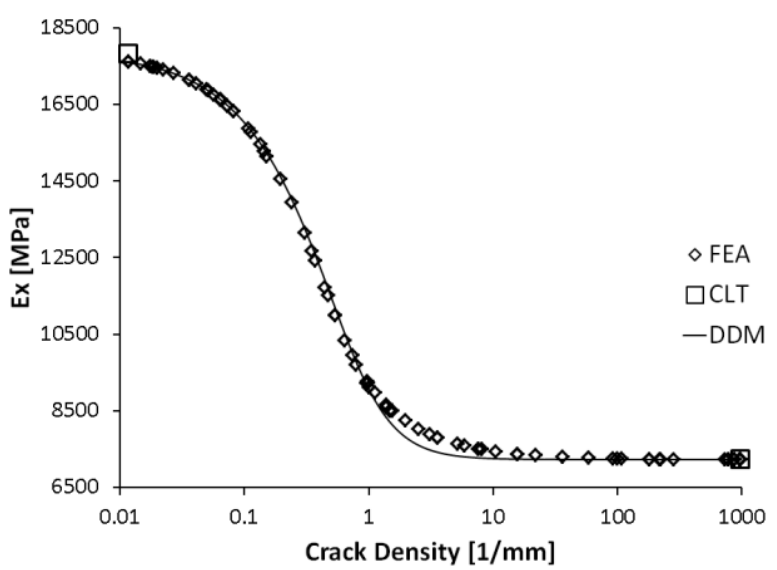

Figure A.1 Ex vs. Crack density for Laminate 2.

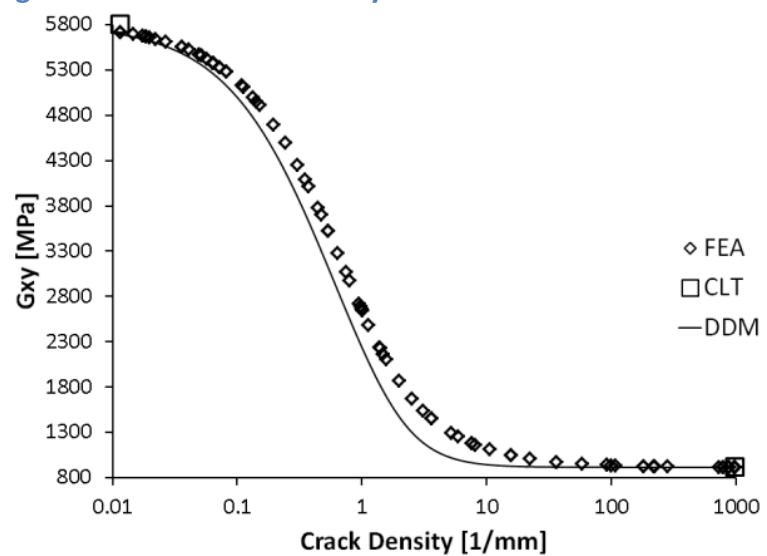

Figure A.3 Gxy vs. Crack density for Laminate 2.

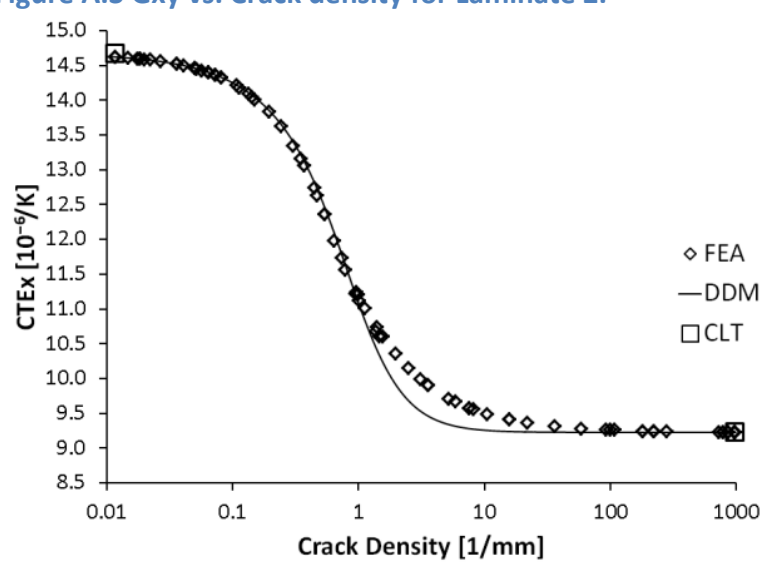

Figure A.5 CTEx vs. Crack density for Laminate 2.

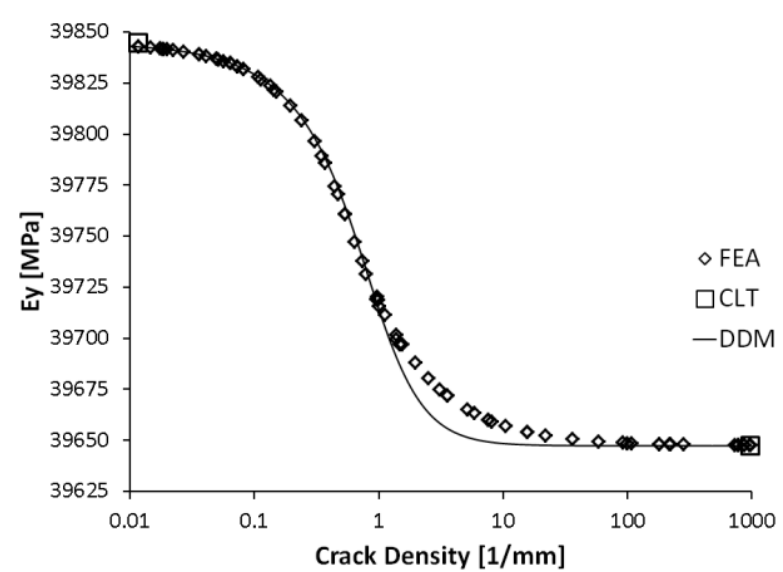

Figure A.2 Ey vs. Crack density for Laminate 2.

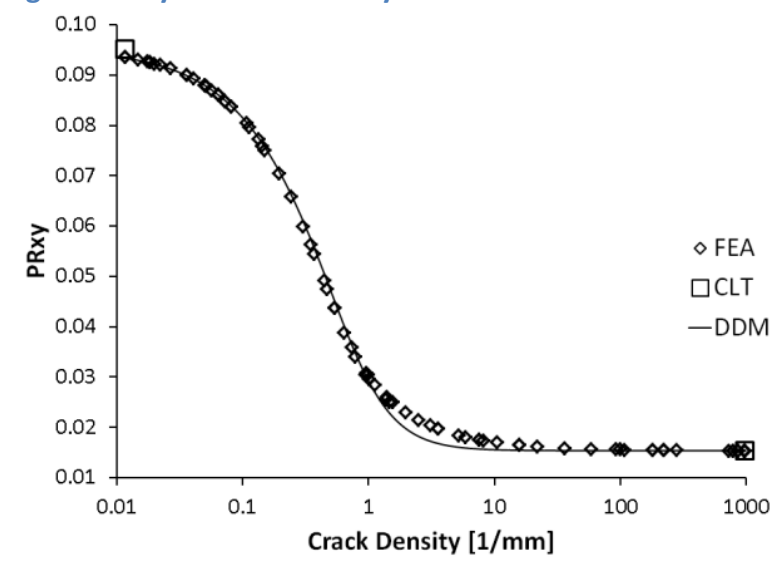

Figure A.4 Poisson's Ration vs. Crack density for Laminate 2.

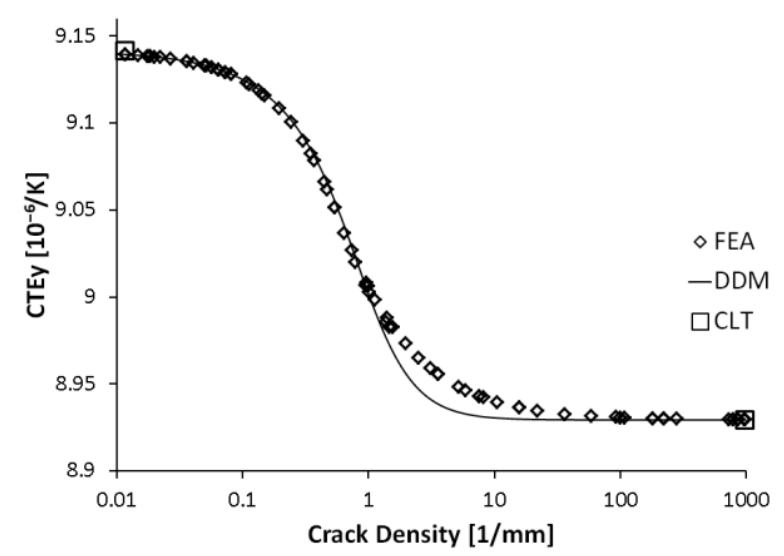

Figure A.6 CTEy vs. Crack density for Laminate 2. 


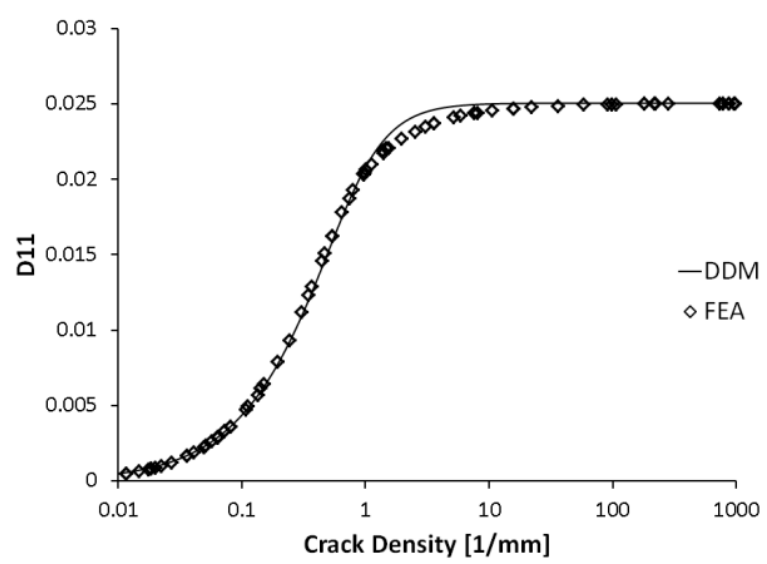

Figure A.7 D11 vs. Crack density for Laminate 2.

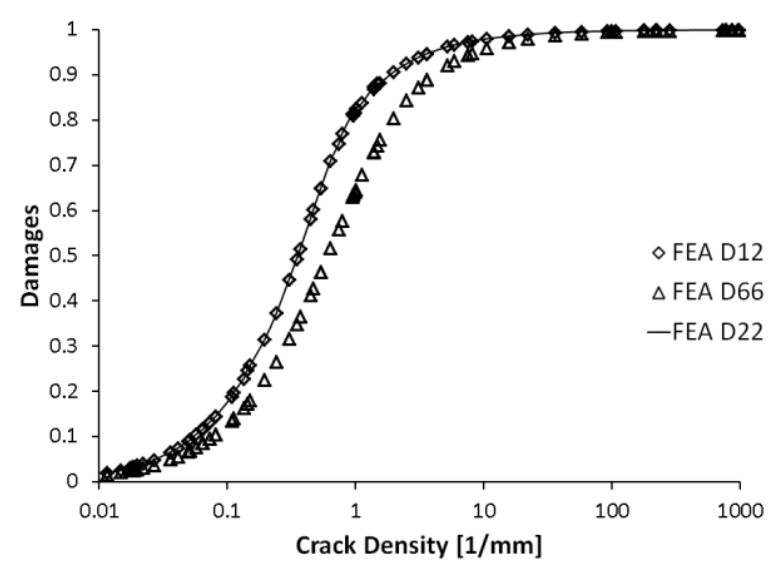

Figure A.8 D12, D66 and D22 vs. Crack density for Laminate 2.

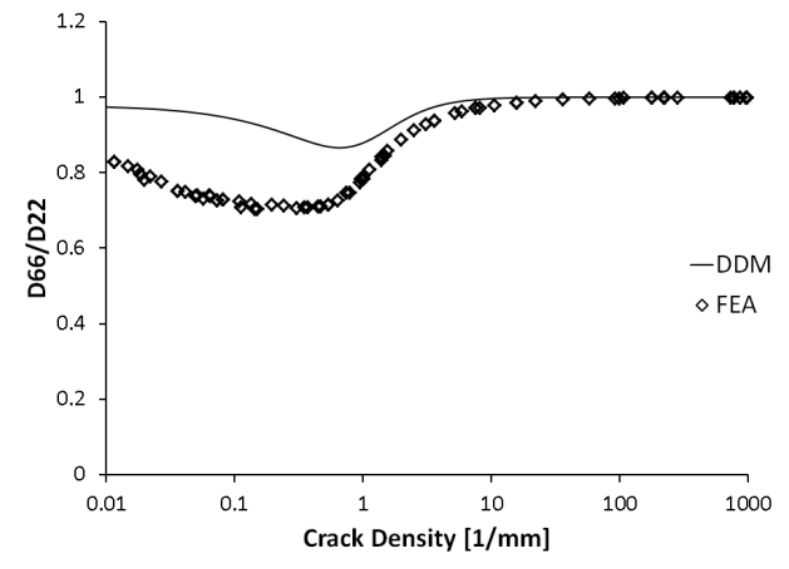

Figure A.9 D66/D22 vs. Crack density for Laminate 2.

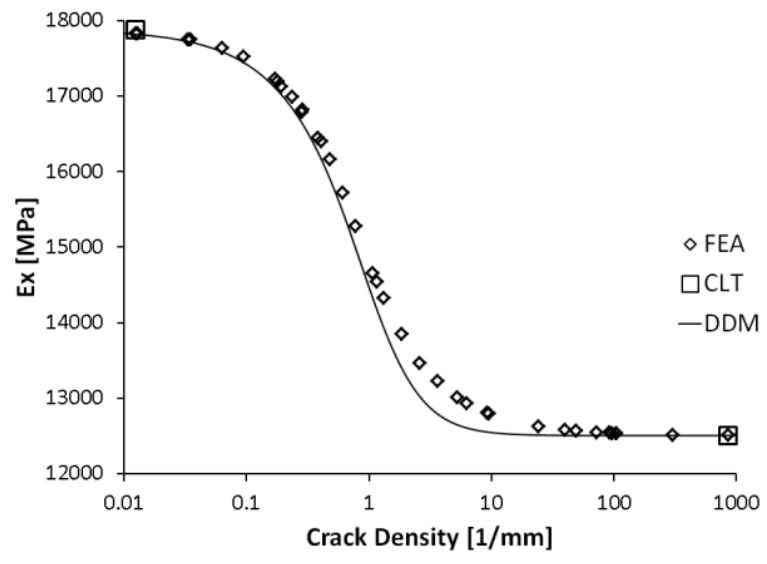

Figure A.10 Ex vs. Crack density for Laminate 3.

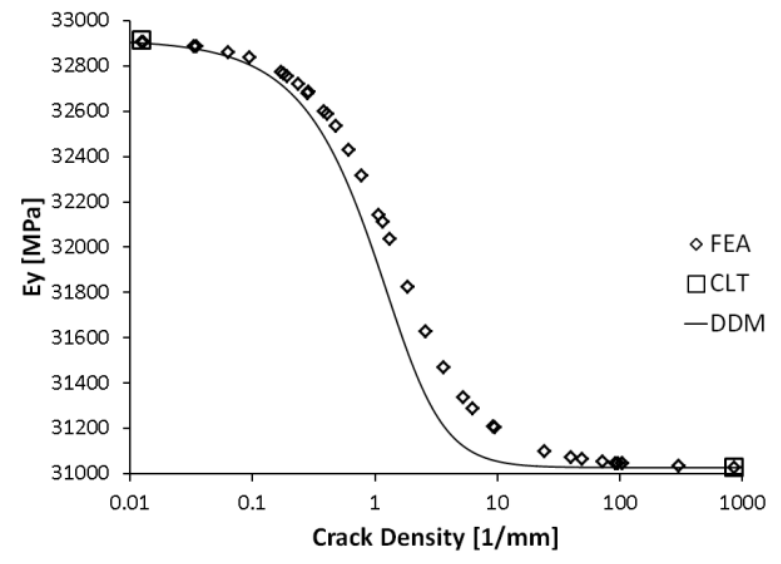

Figure A.11 Ey vs. Crack density for Laminate 3. 


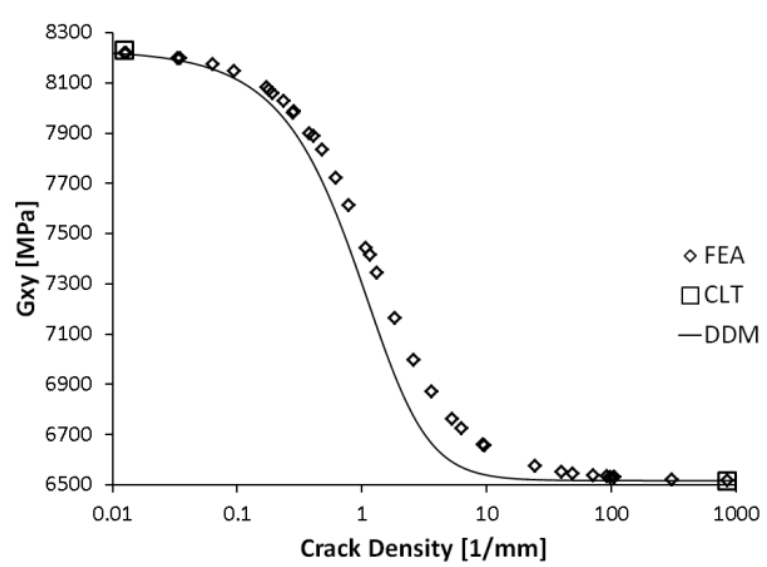

Figure A.12 Gxy vs. Crack density for Laminate 3.

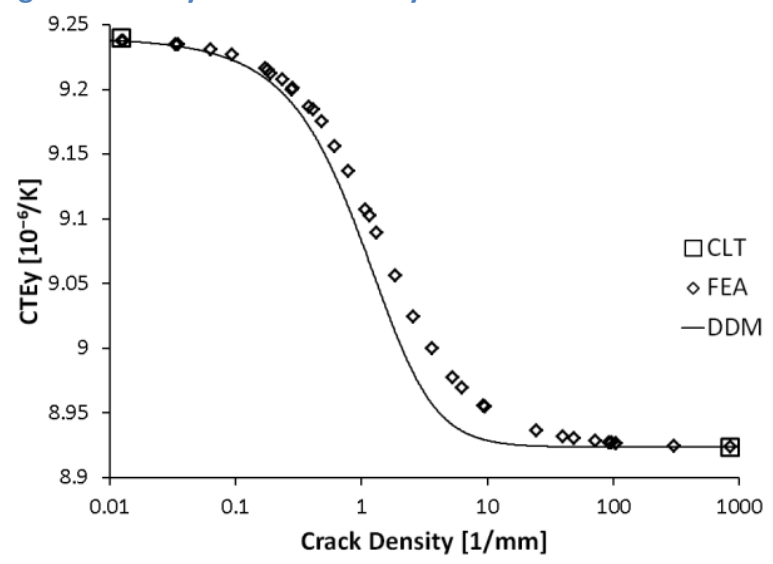

Figure A.14 CTEy vs. Crack density for Laminate 3.

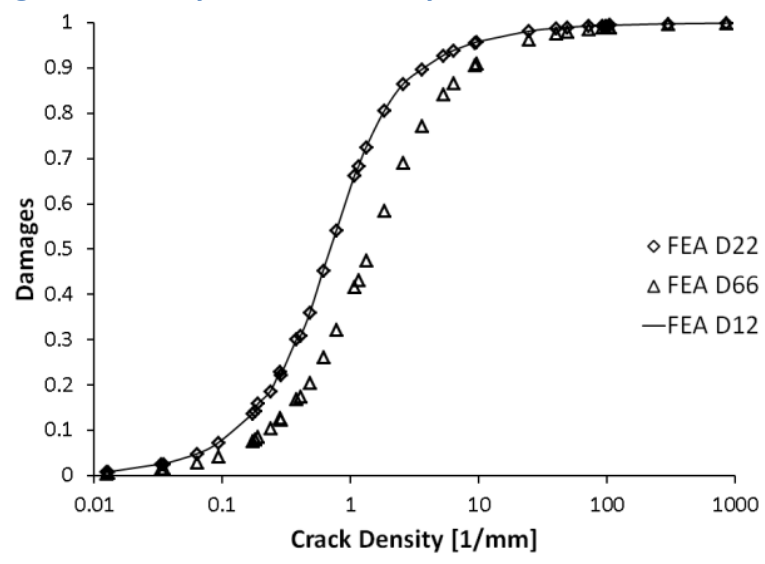

Figure A.16 D22,D66 and D12 vs. Crack density for Laminate 3.

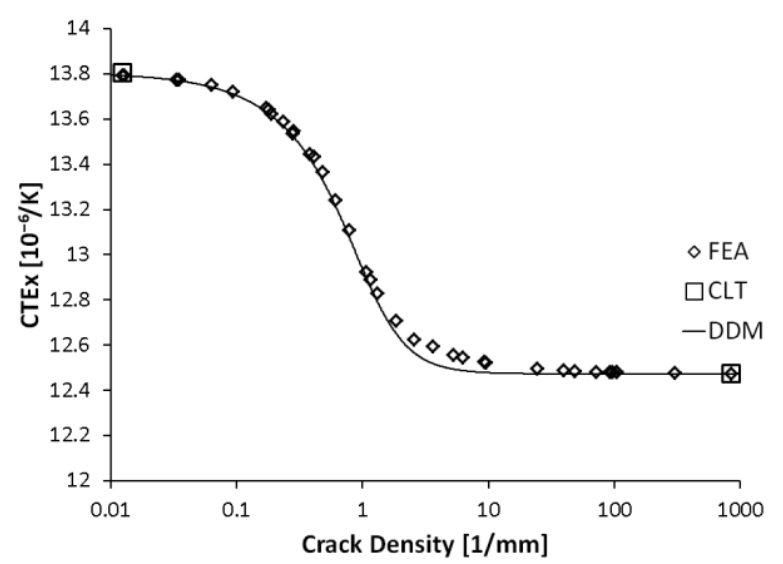

Figure A.13 CTEx vs. Crack density for Laminate 3.

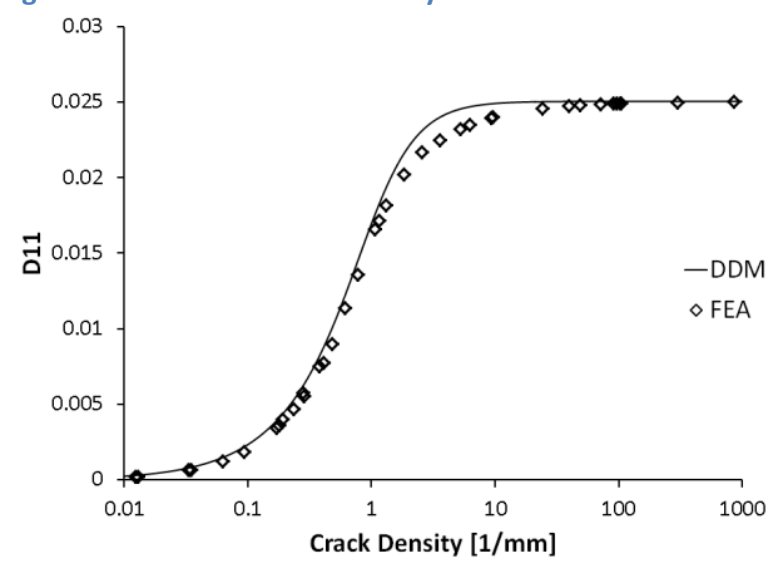

Figure A.15 D11 vs. Crack density for Laminate 3.

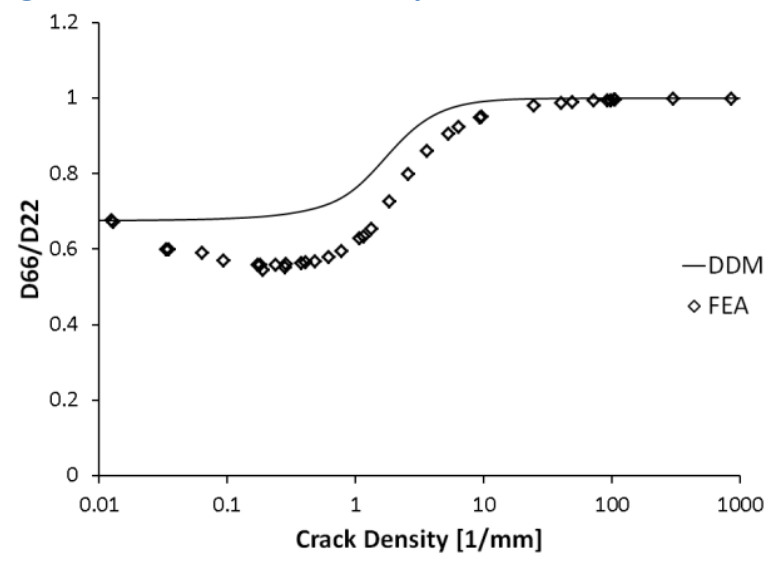

Figure A.17 D66/D22 vs. Crack density for Laminate 3. 

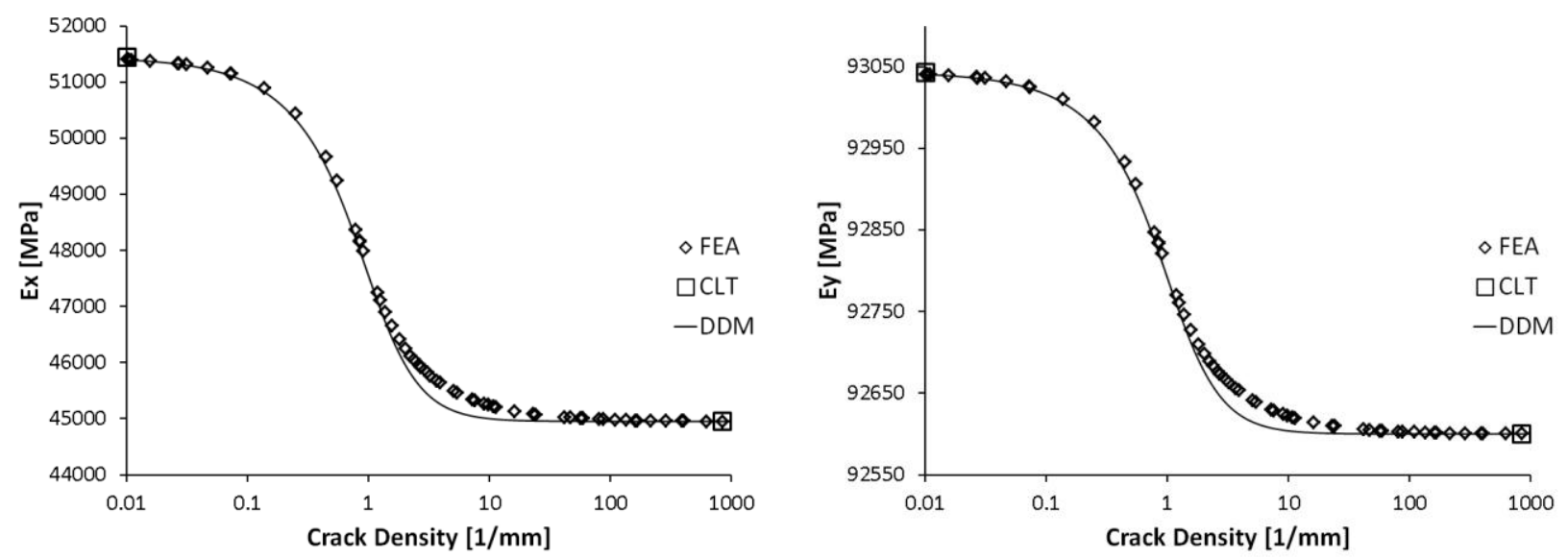

Figure A.18 Ex vs. Crack density for Laminate 4.

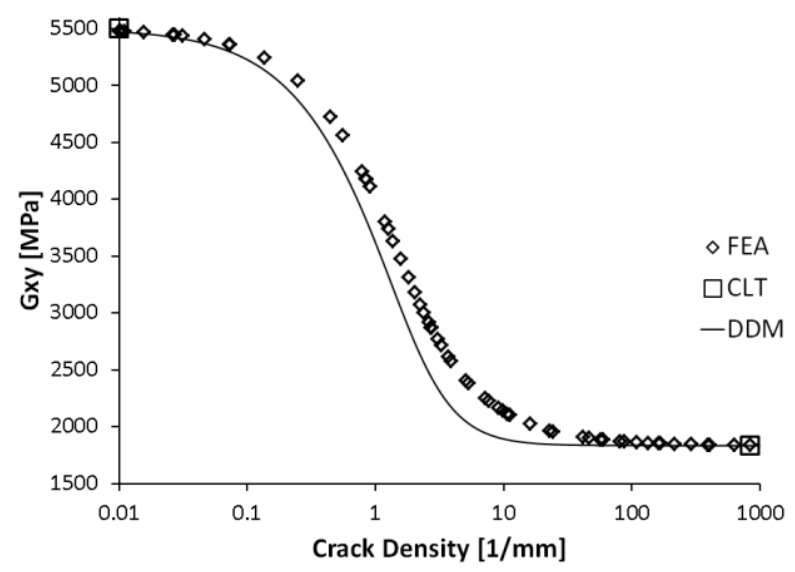

Figure A.19 Ey vs. Crack density for Laminate 4.

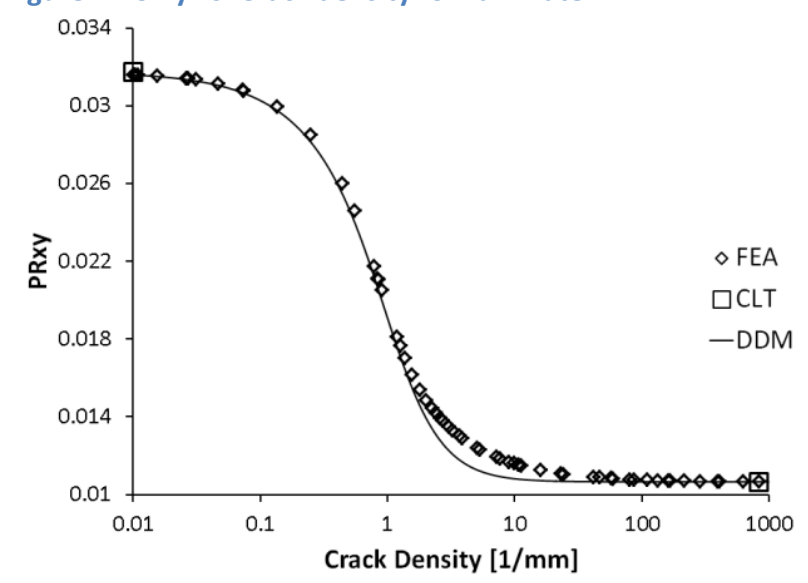

Figure A.20 Gxy vs. Crack density for Laminate 4.

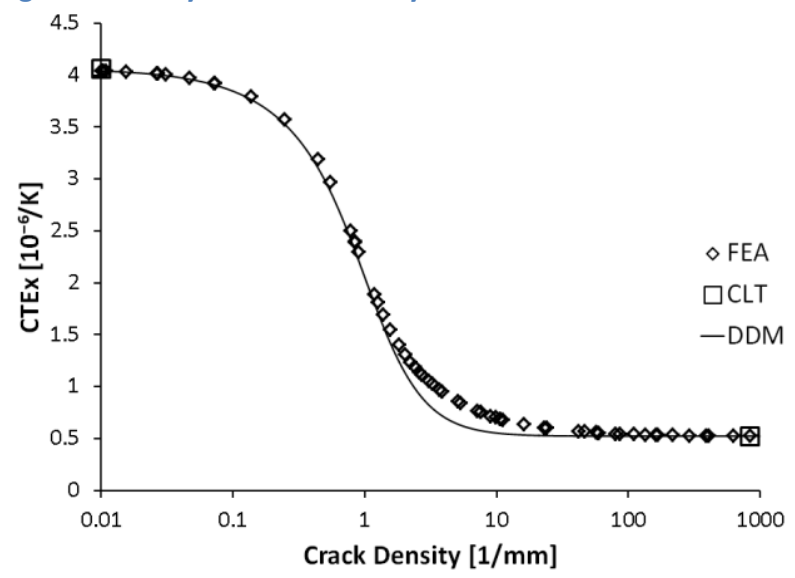

Figure A.21 Poisson's Ration vs. Crack density for Laminate 4.

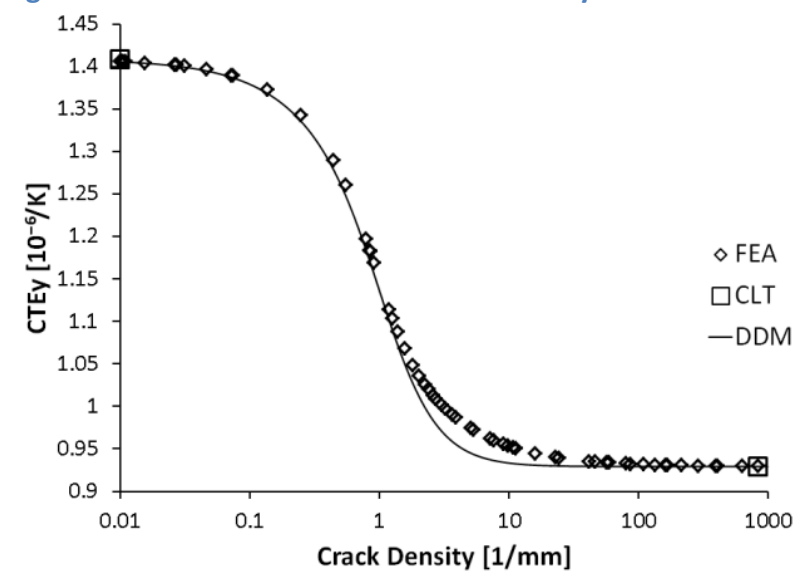

Figure A.22 CTEx vs. Crack density for Laminate 4. 


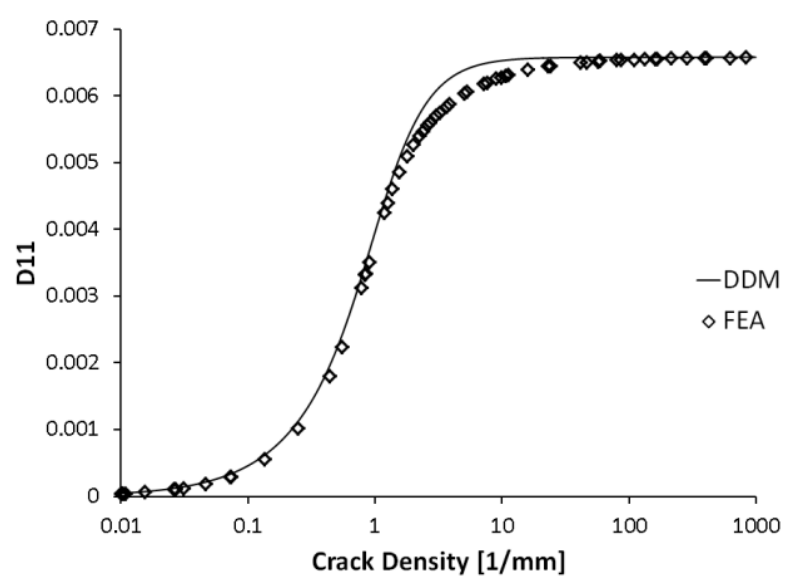

Figure A.24 D11 vs. Crack density for Laminate 4.

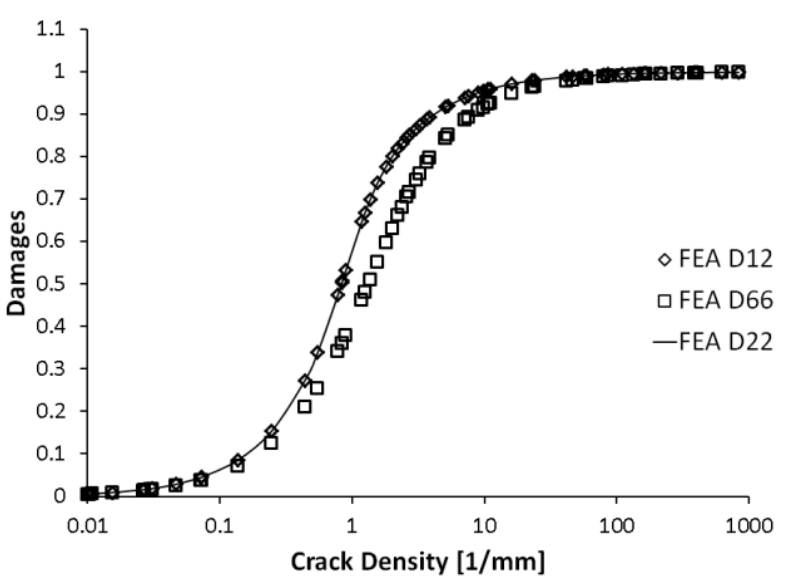

Figure A.25 D12, D66 and D22 vs. Crack density for Laminate 4.

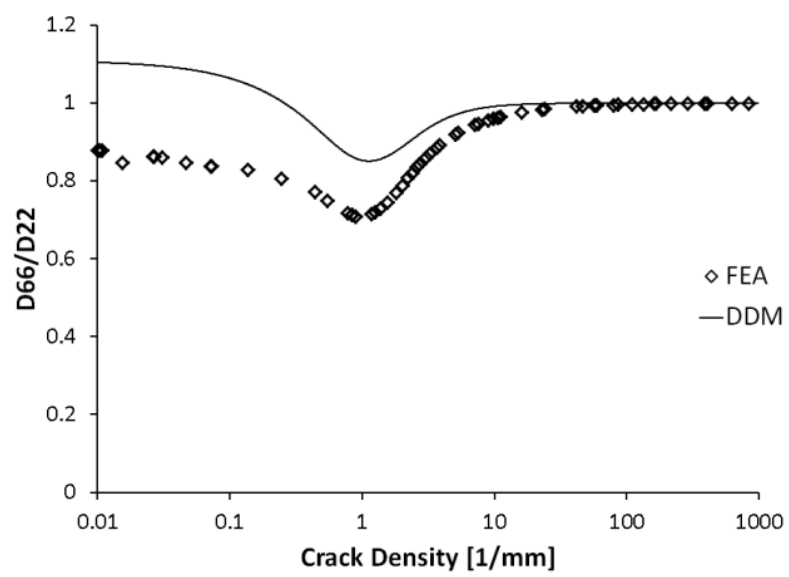

Figure A.26 D66/D22 vs. Crack density for Laminate 4.

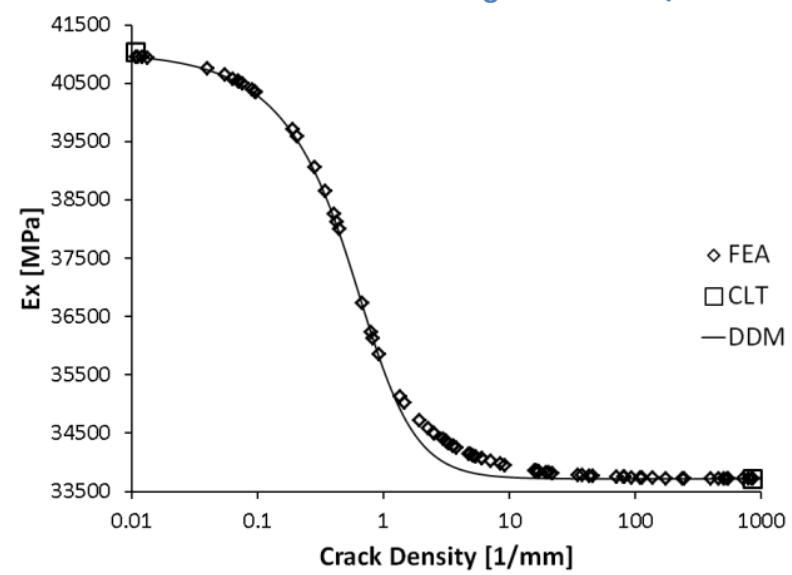

Figure A.27 Ex vs. Crack density for Laminate 5.

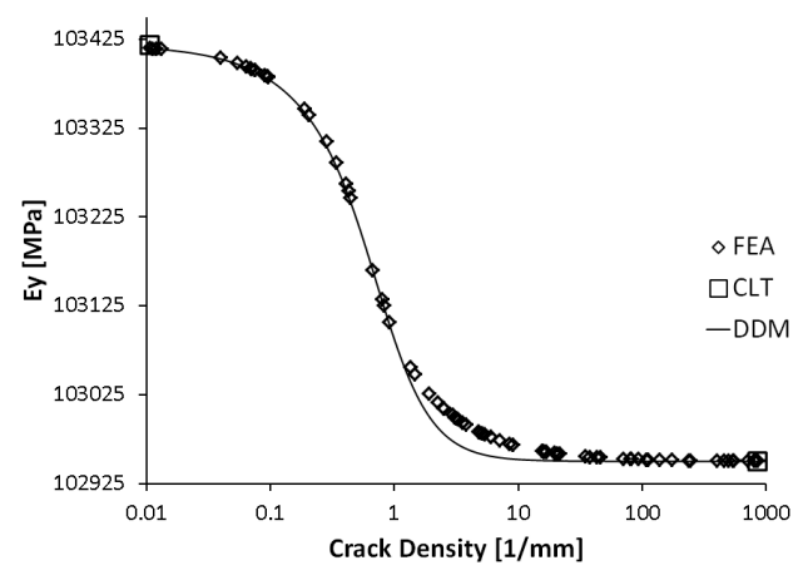

Figure A.28 Ey vs. rack density for Laminate 5. 

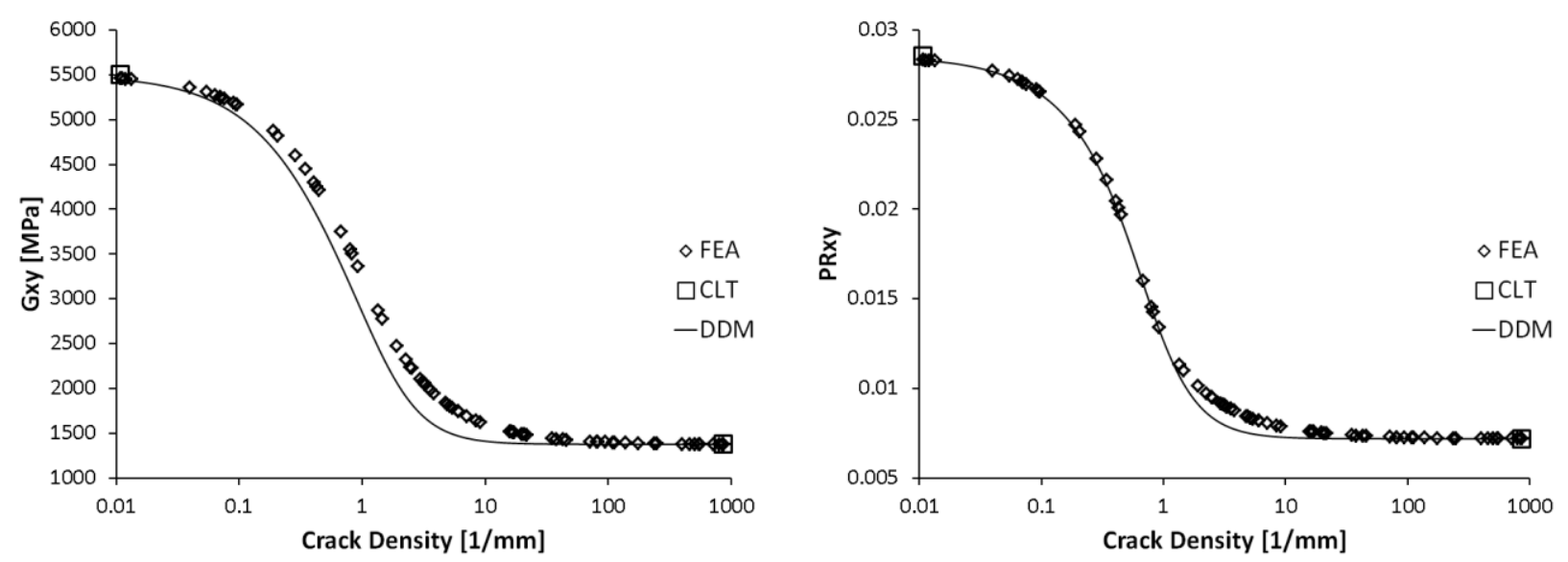

Figure A.29 Gxy vs. Crack density for Laminate 5.

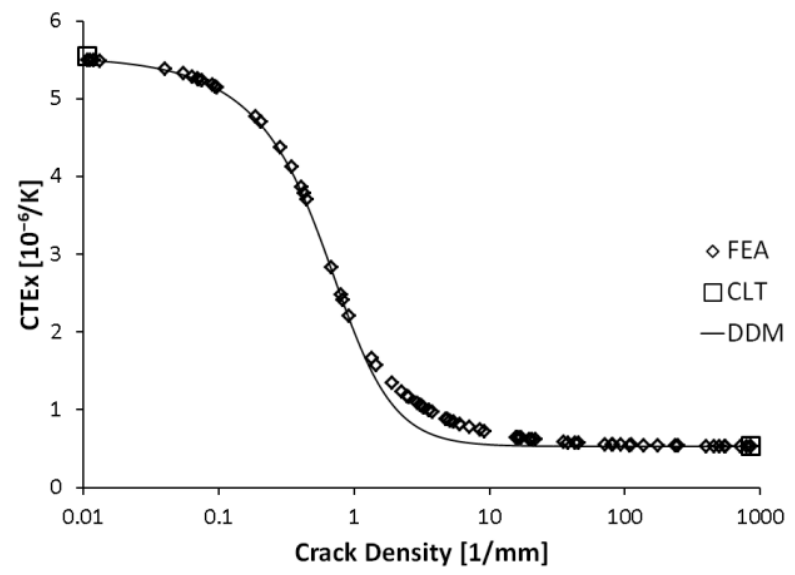

Figure A.30 Poisson's Ratio vs. Crack density for Laminate 5.

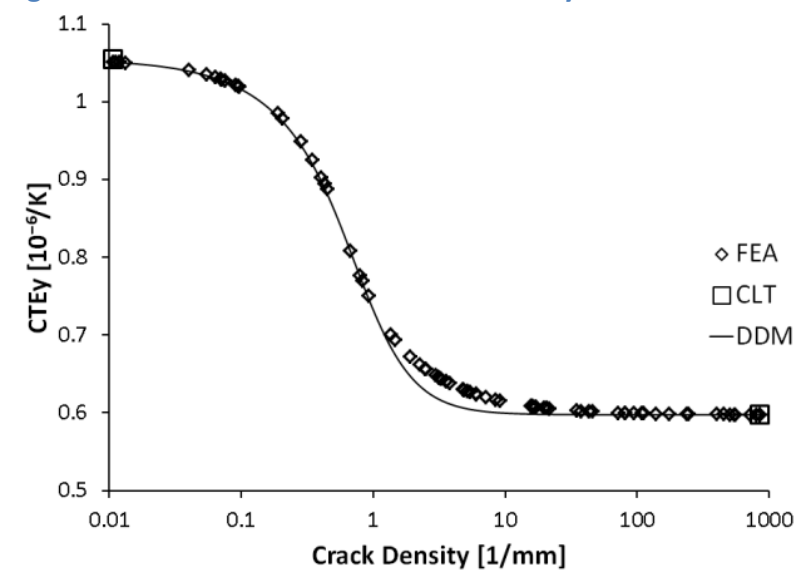

Figure A.31 CTEx vs. Crack density for Laminate 5.

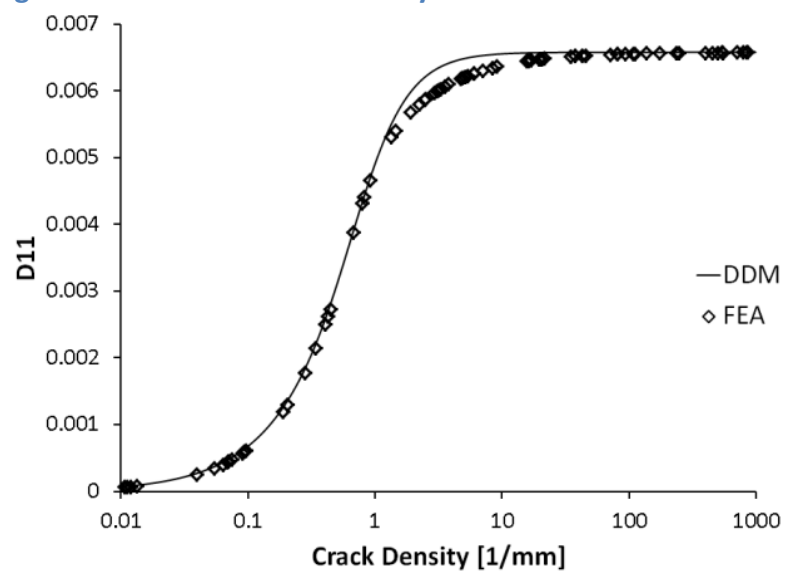

Figure A.33 D11 vs. Crack density for Laminate 5.

Figure A.32 CTEy vs. Crack density for Laminate 5.

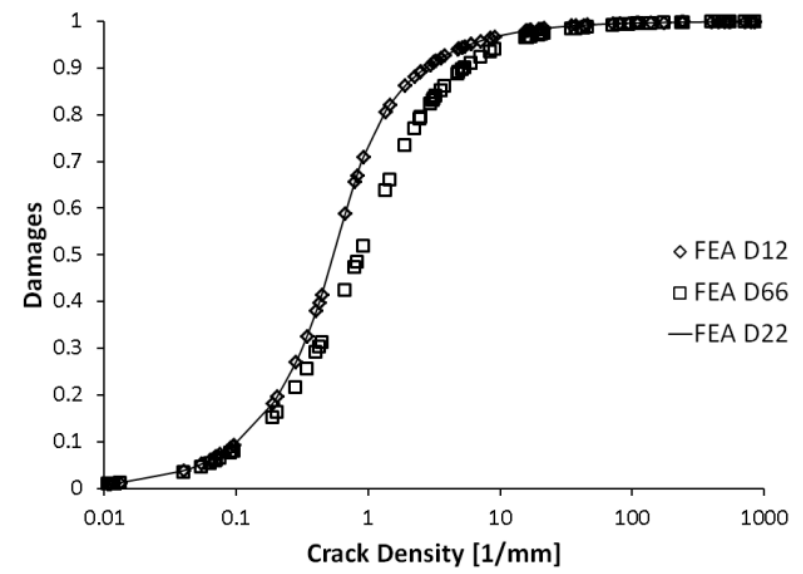

Figure A.34 D12, D66 and D22 vs. Crack density for Laminate 5. 


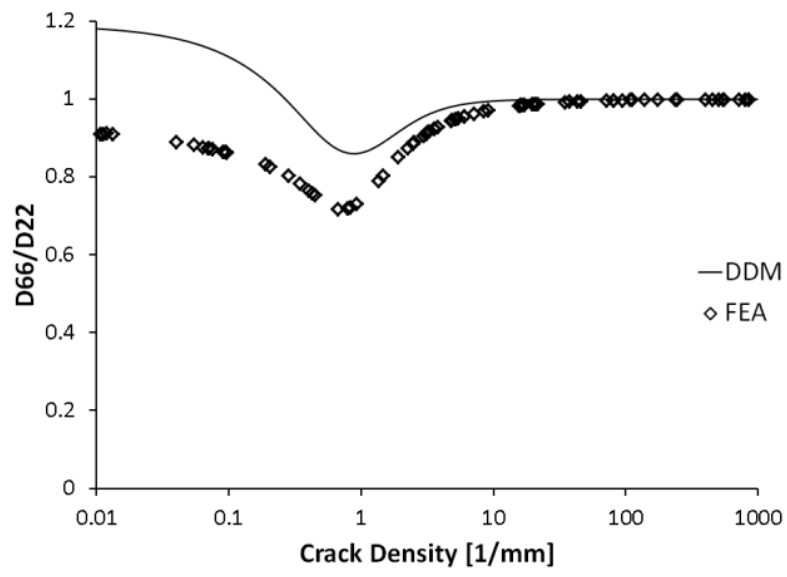

Figure A.35 D66/D22 vs. Crack density for Laminate 5. 